\title{
Earthquakes, Faulting, and Stress in the Los Angeles Basin
}

\author{
EGILL HAUKSSON ${ }^{1}$
}

\author{
Department of Geological Sciences, University of Southem Califomia, Los Angeles
}

\begin{abstract}
Since 1920 fourteen moderate-sized $\left(M_{L}=4.9-6.4\right)$ earthquakes have been reported in the Los Angeles basin. These events are associated with both mappable surficial faults and concealed faults beneath the basin sediments. To determine the style of faulting and state of stress in the basin, single-event focal mechanisms for 244 earthquakes of $M \geq 2.5$ that have occurred during 1977-1989 have been calculated. Fifty-nine percent of the events are strikeslip and are mostly located near two of the major, northwest striking right-lateral strike-slip faults in the basin, the Newport-Inglewood fault and the Palos Verdes fault. The 1988 Pasadena and the 1988 Upland earthquakes showed left-lateral strike-slip on northeast striking faults. Numerous small earthquakes in the eastern part of the basin show left-lateral strike-slip faulting and form a northeast trend near Yorba Linda. Thirty-two percent of the events have reverse mechanisms and are distributed along two broad zones. The first, the Elysian Park fold and thrust belt, coincides with anticlines along the eastern and northern flank of the Los Angeles basin extending into Santa Monica Bay. The second, the TorranceWilmington fold and thrust belt, coincides with anticlines mapped on the southwest flank of the basin and extends from offshore Newport Beach to the northwest into Santa Monica Bay. Oblique faulting that could be inferred by the merging of strike-slip and compressional tectonics does not occur in the basin. Instead, the coexistence of zones of thrusting and large strike-slip faults in the basin suggests that the thrust and strike-slip movements are mostly decoupled. A few normal faulting mechanisms appear to be related to faulting orthogonal to the axes of plunging anticlines. The trend of the maximum horizontal stress varies from $\mathrm{N} 1^{\circ} \mathrm{W}$ to $\mathrm{N} 31^{\circ} \mathrm{E}$ across the basin and consistently forms high angles with the fold axes. The stress field that exists along the flanks of the basin has a vertical minimum stress axis. This stress field and ongoing folding and thrusting suggest that tectonic deformation is concentrated along the flanks of the deep central basin. Today the deformation of the basin consists of uplift and crustal thickening and lateral block movement to accommodate the north-south compression across the basin.
\end{abstract}

\section{INTRODUCTION}

The Los Angeles basin is one of several deep Cenozoic basins that formed on the margin of the Pacific plate, near the San Andreas fault in southern California. Located at the northern edge of the Peninsular Ranges, it abuts the southern margin of the central Transverse Ranges (Figure 1). Today the Los Angeles basin consists of a deep central basin with marine and fluvial sediment up to $10 \mathrm{~km}$ thick and folded and uplifted east, north, and southwest flanks [Yerkes et al., 1965; Davis et al., 1989]. This study analyzes earthquake data from the Los Angeles basin proper and the adjacent offshore Santa Monica Bay and San Pedro Bay and the San Gabriel Valley.

The 1987 ( $M_{L}=5.9$ ) Whittier Narrows earthquake occurred on a concealed thrust fault beneath the folded sediments of the north flank of the basin [Davis et al., 1989; Hauksson and Jones, 1989]. This study attempts to determine where similar zones of folding and thrust faulting exist in the Los Angeles basin. Identifying such zones of

\footnotetext{
${ }^{1}$ Now at Seismological Laboratory, Division of Geological and Planetary Sciences, California Institute of Technology, Pasadena.

Copyright 1990 by the American Geophysical Union.

Paper number $90 \mathrm{JB} 00807$. 0148-0227/90/90JB-00807\$05.00
}

thrusting is important because they could cause similar or even more damaging earthquakes than the 1987 Whittier Narrows earthquake.

Data from moderate-sized events $(M \geq 4.9)$ since 1920 and small earthquakes $2.5 \leq M<4.9$ since 1977 throughout the Los Angeles basin have been analyzed to evaluate active faulting in the basin. A map of the epicenters of earthquakes recorded between January 1973 and May 1989 in the greater Los Angeles basin shows events scattered throughout with no simple spatial patterns (Figure 2). This is in contrast to the clustering of earthquakes along major faults observed in many other parts of California such as along the San Jacinto fault [Allen et al., 1965; Sanders, 1986]. Thus the spatial distribution of seismicity alone cannot be used to map subsurface expressions of active faults in the Los Angeles basin.

Single-event focal mechanisms have been determined for 244 earthquakes of $2.5 \leq M \leq 5.9$ in the Los Angeles basin. Although the spatial distribution of epicenters does not delineate specific faults, the focal mechanisms do form systematic spatial patterns. These patterns are used to evaluate the spatial extent of strike-slip, normal, and thrust or reverse faulting.

The Los Angeles basin forms a transition zone between the strike-slip tectonics of the Peninsular Ranges and the convergent tectonics of the Transverse Ranges. Both strike-slip and compressional tectonic structures exist within this transition zone. Traditionally, the compressive structures such as folds have been interpreted as secondary 


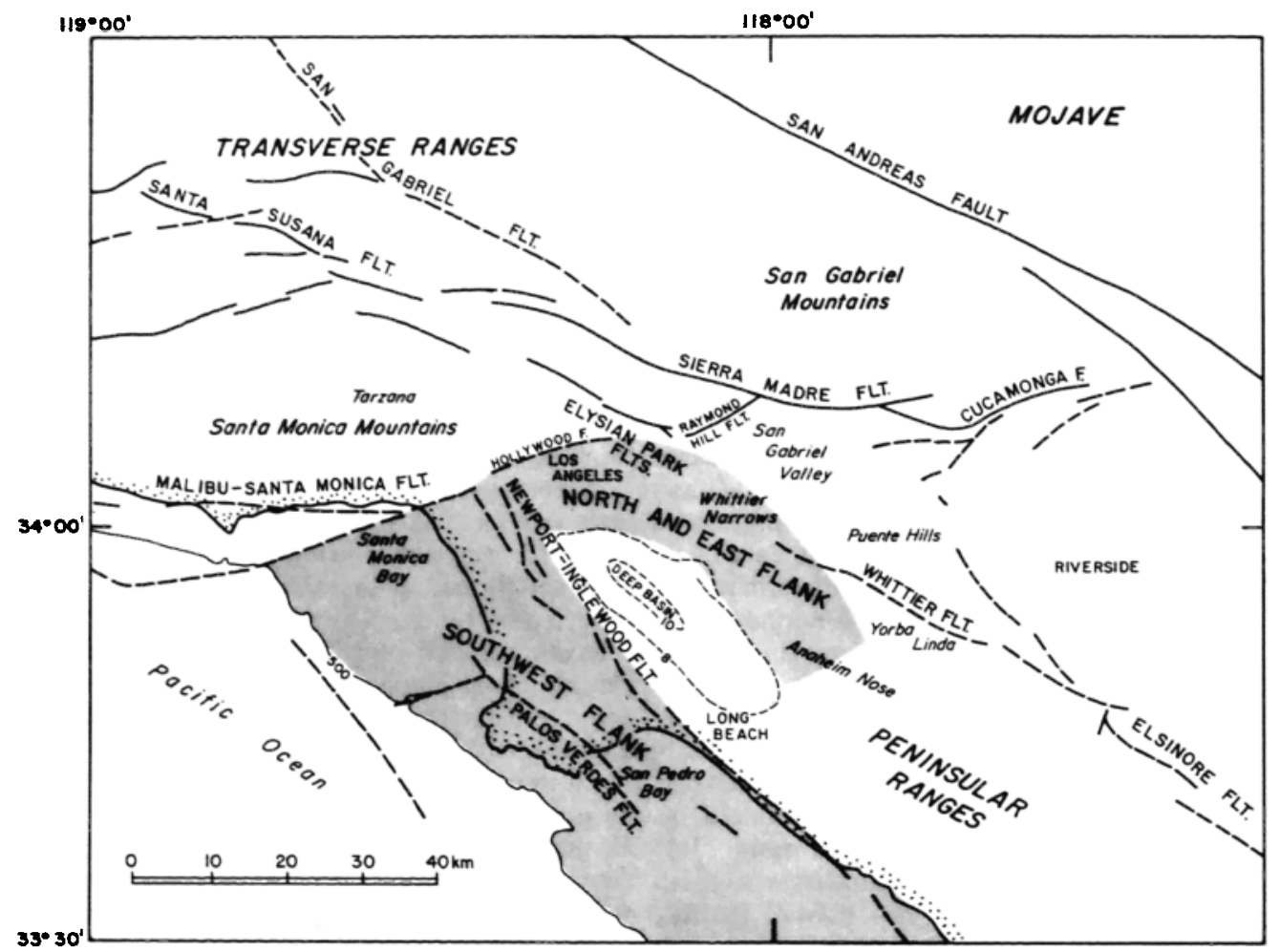

Fig. 1. Map showing geographical features, late Quaternary faults, and the Los Angeles basin in southern California. The flanks of the basin as defined in this study are shown as shaded areas. The east flank is defined as as the region in between the $8 \mathrm{~km}$ sediment depth contour of the basin [Yerkes et al., 1965] and the Puente Hills to the east. The north flank is defined as the area between the $8 \mathrm{~km}$ depth contour and the Santa MonicaHollywood faults to the north. The southwest flank is defined as the area between the $500 \mathrm{~m}$ bathymetric contour offshore and the Newport-Inglewood fault. The 8 and $10 \mathrm{~km}$ depth contours outline the deep central basin.

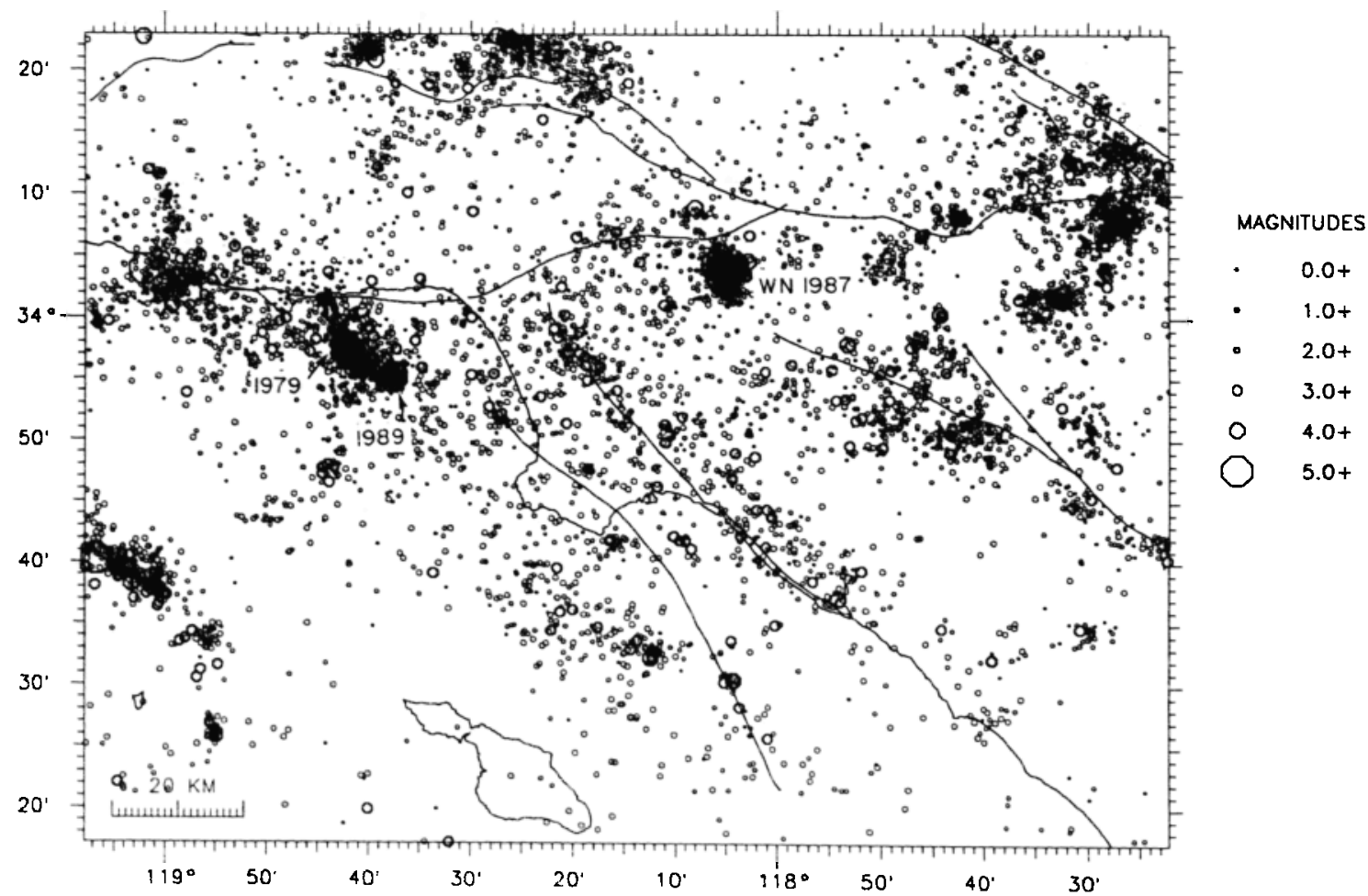

Fig. 2. Seismicity in the Los Angeles basin from 1973 to 1989 recorded by the CIT/USGS Southem California Seismic Network and the USC Los Angeles Basin Seismic Network. The 1979 and 1989 indicate the location of the two mainshock-aftershock sequences in Santa Monica Bay. WN 1987 indicates the 1987 Whittier Narrows sequence. 
structures caused by movements on the primary late Quaternary strike-slip faults. This interpretation is called the wrench tectonics model [e.g., Wilcox et al., 1973]. The wrench faulting models assume layers of soft sediments that deform in response to motion on a strikeslip fault at depth below them. The Newport-Inglewood fault on the southwest flank of the basin is often presented as the classic example of wrench faulting [Wilcox et al., 1973]. Recently, Wright [1987] has pointed out the strong disagreement between the actual distribution and orientation of fold axes along the Newport-Inglewood and Whittier faults and those predicted by the wrench faulting model. Similarly, the folding along the north flank of the basin cannot be associated with a known strike-slip fault. Thus the folding along the flanks of the basin cannot easily be explained as secondary effects from strike-slip faulting.

If both the strike-slip and compressional tectonics in the basin are of similar importance, their coexistence could suggest that the faulting in the basin should be mostly oblique. Alternatively, the merging of these two tectonic styles could take place as decoupled strike-slip and thrust faulting. Such decoupling occurred on a smaller scale in the 1987 Whittier Narrows sequence where the mainshock ruptured a gently dipping thrust fault and the largest aftershock ruptured a steeply dipping strike-slip fault [Hauksson and Jones, 1989]. One of the goals of this study is to explore the possible role of decoupling of fault slip in the tectonic deformation of the basin.

The relative importance of strike-slip and compressional tectonics is also evaluated by determining the present stress field. The focal mechanisms are used in this study to determine the orientations of the three principal stress axes and their relative magnitudes. Compressional structures such as folds are finite strain markers, which reflect the stress field at the time of formation. In this study the relationship between the present stress field and orientation of fold axes are analyzed. If the stress field had changed since the formation of the folds, the orientation of fold axes could be expected to be in part inconsistent with the present stress field. Furthermore, within regions of strikeslip faulting the intermediate principal stress is vertical, while within regions of thrust faulting the minimum principal stress is vertical. If strike-slip and thrust faulting are of similar importance in the basin, the intermediate and minimum principal stresses should be of very similar magnitude and hence could interchange in orientation depending on local perturbations in the stress field.

In addition to the $1987\left(M_{L}=5.9\right)$ Whittier Narrows earthquake, numerous moderate-sized or large earthquakes have shown that folds located above thrust faults can grow during the earthquake. The $1980\left(M_{S}=7.3\right)$ El Asnam, Algeria, earthquake [Nabelek, 1985], the $1983\left(M_{S}=6.5\right)$ Coalinga earthquake [Eaton, 1985; Namson and Davis, 1988], and the $1985\left(M_{S}=6.7,6.9\right)$ Nahanni, Canada, earthquakes [Wetmiller et al., 1988] all occurred on concealed thrust faults and caused coseismic growth of surficial folds. Although numerous earthquakes that have occurred on concealed thrust faults have been documented in detail, no simple methods exist for estimating the maximum size of an earthquake on a buried thrust fault. One of the largest instrumentally recorded earthquakes to occur on a thrust fault in California was the 1952
( $\left.M_{S}=7.7\right)$ Kern County earthquake. It was caused by rupture on a $75-\mathrm{km}$-long concealed thrust fault near the southern end of the San Joaquin Valley [Stein and Thatcher, 1981]. The surface rupture that was reported along the White Wolf fault poorly fits the geodetic data [Stein and Thatcher, 1981] and may have been secondary faulting. If the thrust faults beneath the flanks of the basin are capable of generating similar earthquakes, the earthquake hazards in the Los Angeles basin have been underestimated in the past because estimates of the earthquake potential of these thrust faults have not been included [e.g., Ziony and Yerkes, 1985].

\section{Tectonic Seiting}

The Los Angeles basin has gone through several episodes of tectonic deformation since it began forming in the middle Miocene (16-11 Ma) [Davis et al., 1989]. The inception of the basin probably included volcanism, block rotation, and extensional block faulting on northwest to north striking faults [Campbell and Yerkes, 1976; Wright, 1987]. The Newport-Inglewood fault zone may already have been a zone of weakness during this time, because its southwest flank was uplifted prior to middle Miocene (Figure 1) [Wright, 1987]. Toward the end of middle Miocene, Tarzana and Puente fan deposition initiated. Today sediment from the Santa Monica Mountains have replaced the flux of sediment from the Tarzana fan. Some of the old Tarzana fan sediment outcrop in the Elysian Hills north of downtown Los Angeles. Sediment from the Puente fan, however, continue to be deposited into the basin through the Whittier Narrows [Wright, 1987].

In late Miocene and earliest Pliocene (8-4 Ma), the Los Angeles basin went through its main phase of subsidence and deposition and acquired its present form [Yerkes et al., 1965]. During this period, the sedimentation was primarily turbidity currents travelling down to the basin floor at 1-2 km depth below sea level [Yerkes et al., 1965]. Toward the end of this time, the basin filled more rapidly than it subsided. Concurrently, left-lateral faulting along the southem margin of what is now the east-west trending Transverse Ranges dominated deformation in the north Los Angeles basin [Lamar, 1961].

The opening of the Gulf of California in late Pliocene time (4-2 Ma) (Pasadenan deformation) had a profound effect on the neotectonics of southern California [Atwater, 1989; DeMets et al., 1987]. In the Los Angeles basin, the type of deformation changed from northwest-southeast extension to north-south compression [Wright, 1987]. Since the onset of compressional tectonics, the upper Pliocene sediments have been uplifted, folded, and in some cases overturned by the north-south compressive stress field [Yerkes et al., 1965].

Today the Los Angeles basin includes the deep basin and east, north, and southwest flanks (Figure 1). The deep basin forms a northwest-southeast elongated synclinorium filled with marine and fluvial sediments, while the flanks are uplifted and folded. Using the retrodeformable crosssection technique, Davis et al. [1989] interpreted compressional structures along two profiles crossing the basin. One profile crosses the middle of the basin, along a north-south line, and another profile provides a detailed structural interpretation of the Whittier Narrows area. Both 
of these profiles, constructed using the fault-fold relationships technique of Suppe [1985], model the nearsurface folding as caused by thrust faulting at depth. Thus Davis et al. [1989] infer thrust faults at depth, although they are not able to tell if these faults are seismogenic at present.

In addition to the compressional geologic structures, the basin is also crosscut by northwest to north-northwest striking right-lateral strike-slip faults of late Quaternary age including the Whittier fault, Newport-Inglewood fault, and the Palos Verdes fault. The northern margin of the basin is bounded by the range front faults, such as the Santa Monica, Hollywood, and Sierra Madre faults [Crook et al., 1987; Morton and Yerkes, 1987]. Some of these exposed late Quatemary faults may be seismogenic, while others are thought to be mostly aseismic [Ziony and Yerkes, 1985].

\section{EARTHQUAKE Data AND ANALYsis}

Earthquakes occurring within the area extending from latitude $33^{\circ} 26^{\prime} \mathrm{N}$ to $34^{\circ} 16^{\prime} \mathrm{N}$ and from longitude $117^{\circ} 28^{\prime} \mathrm{W}$ to $118^{\circ} 48^{\prime} \mathrm{W}$ were analyzed in this study (Figure 2). The earthquake data from 1977 to 1989 were recorded by both the California Institute of Technology/U.S. Geological Survey (CIT/USGS) Southern California Seismic Network and the University of Southern California (USC) Los Angeles Basin Seismic Network. Digital seismograms of all 244 earthquakes of $M \geq 2.5$ discussed in this study were analyzed either specifically for this study or for other studies [Hauksson, 1987; Hauksson and Saldivar, 1989; Hauksson and Jones, 1989]. Earthquakes from two periods (May 1980 to March 1981 and February to June 1983) are not included because the CIT/USGS digital seismic data have not yet been processed.

Four sets of velocity models and station delays were used to calculate hypocenters and takeoff angles in this study. These models were determined by inverting $P$ and $S$ arrival time data with the VELEST computer program [Roecker and Ellsworth, 1978]. In the east Los Angeles basin and along the Newport-Inglewood fault the models and delays derived by Hauksson and Jones [1989] and Hauksson [1987] were used, respectively. Events located offshore in Santa Monica Bay were located using models derived by Hauksson and Saldivar [1989]. As a part of this study a new set of models and delays were derived for the San Pedro Bay area. Final locations were calculated using HYPOINVERSE [Klein, 1985] and the velocity models and station delays for the respective epicentral region. A distance weight of one in the distance range $0-100 \mathrm{~km}$ and linearly decreasing weight from one to zero in the distance range 100-120 km was applied. In most cases both the epicenter and the focal depth are constrained to better than $1 \pm 1 \mathrm{~km}$. For events located offshore, however, the focal depths are less certain.

The 244 single-event, lower hemisphere focal mechanisms were determined from $P$ wave first motion polarities using a grid-searching algorithm and computer programs by Reasenberg and Oppenheimer [1985]. To avoid possible bias in the state of stress from major aftershock sequences, only the focal mechanisms of the mainshock and largest aftershock for $M \geq 5.0$ mainshocks are included in this study. No systematic effort was made to exclude aftershocks of $M<5.0$ mainshocks because these are usually followed by few if any aftershocks of $M \geq 2.5$. In Figures 3, 4, and 5, depending on the rake for the selected plane, the focal mechanisms have been grouped into strike-slip, normal, and thrust faulting, respectively. Normal mechanisms have rakes ranging from $-45^{\circ}$ to $-135^{\circ}$. Thrust mechanisms have rakes ranging from $45^{\circ}$ to $135^{\circ}$. Five events with nodal planes dipping less than $20^{\circ}$ and rakes close to $0^{\circ}$ or $180^{\circ}$ are also classified as thrust faulting. Strike-slip events have rakes ranging from $44^{\circ}$ to $44^{\circ}$ and $136^{\circ}$ to $224^{\circ}$. In most cases the grouping is clear, although in a few cases the event may be strike-slip and normal or thrust depending on which plane is selected. If the first motion data could be fit with more than one solution, the other solutions are also shown and are flagged with a star.

A stress inversion technique developed by Michael [1984] was used to invert the focal mechanisms for the state of stress. The inversion minimizes the misfit angle (B) between the direction of the shear stress on the fault plane and the observed slip direction on that plane determined from focal mechanism. The inversion assumes that the regional stress field is a constant tensor, all slip events are independent, and the magnitude of the tangential traction $(|T|)$ applied to each fault plane is similar. The third assumption is equivalent to assuming that $|T|=1$, because only relative stress magnitudes can be calculated. The inversion solves for the orientation of the three principal stress axes and the $\varnothing$ value, a measure of the relative magnitude of the principal stresses defined as

$$
\phi=\left(S_{2}-S_{3}\right) /\left(S_{1}-S_{3}\right)
$$

where $S_{1}, S_{2}$, and $S_{3}$ are the maximum, intermediate, and minimum compressive principal stresses.

One plane must be selected from each focal mechanism as the actual fault plane [Michael, 1987a]. In this study, the planes listed in Table 1 are those selected for the inversions. In general, north to northwest striking rightlateral planes in strike-slip focal mechanisms were chosen. In a few instances, where the strike-slip mechanisms align along a northeast trend, the east to northeast striking planes were chosen. The north dipping planes in the reverse or thrust mechanisms were chosen because these are assumed to be more consistent with the geological deformation than the south dipping planes [Davis et al., 1989]. In San Pedro Bay, however, the south dipping planes were chosen for the thrust events because the trend of hypocenters appears to dip to the south. The planes indicating faulting down into the Los Angeles basin were chosen for the normal faulting mechanisms.

Although the geological information makes it possible to select some planes correctly, other planes may be picked incorrectly. This is accounted for in the bootstrap technique used to calculate the confidence limits by assuming that $30 \%$ of the planes are picked incorrectly [Michael, 1987b].

\section{EARTHQUAKES}

Since 1920,14 moderate-sized $\left(M_{L}=4.9-6.4\right)$ mainshockaftershock sequences have occurred in the greater Los Angeles basin (Figures $6 a$ and $6 b$ and Table 2). These earthquakes do not occur along a single fault but are 
associated with many low slip-rate, late Quaternary faults that are distributed over a large area [Ziony and Yerkes, 1985; Ziony and Jones, 1989]. Research on Los Angeles basin earthquakes was initiated by Taber [1920], who was commissioned by the Seismological Society of America to study the 1920 Inglewood $\left(M_{L}=4.9\right)$ earthquake. He attributed the earthquake to slip on the Newport-Inglewood fault just west of the town of Inglewood, because the event caused concentrated heavy damage in the town.

The three largest historic earthquakes that have caused the most damage in the Los Angeles area are the 1933 $\left(M_{L}=6.3\right)$ Long Beach, $1971\left(M_{L}=6.4\right)$ San Fernando, and $1987\left(M_{L}=5.9\right)$ Whittier Narrows earthquakes. The 1933 Long Beach earthquake occurred on the southem segment of the Newport-Inglewood fault, from Newport Beach to Signal Hill, and showed right-lateral strike-slip movement [Wood, 1933; Richter, 1958; Woodward-Clyde Consultants, 1979]. The 1971 San Fernando earthquake showed reverse faulting with a component of left-lateral strike slip and ruptured along the San Fernando member of the Sierra Madre fault zone [Whitcomb et al., 1973; Heaton, 1984]. The 1987 Whittier Narrows earthquake, which occurred on a previously unrecognized concealed thrust fault, showed pure thrust motion on a gently north dipping plane [Hauksson and Jones, 1989]. Only three focal mechanisms in Figure $6 b$ from a data set of nine $\left(M_{L} \geq 4.9\right)$ events show mostly strike-slip movement, while six events show mostly reverse or thrust faulting. The focal mechanisms of the moderate-sized earthquakes reflect the local geology but are too few to resolve the details of the boundary between the thrust regime to the north and the strike-slip regime to the south.

The rate of small earthquakes in the Los Angeles basin and the adjacent offshore areas is similar to the average rate for southem California [Allen et al., 1965]. In Figure 2, all the earthquake locations in the CIT/USGS catalog from January 1973 to May 1989 are plotted. Since 1973 the dense CIT/USGS and USC networks of seismograph stations have been in operation in and around the Los Angeles basin, providing excellent coverage of seismic activity. The densest clusters of events in Figure 2 are mainshock-aftershock sequences such as 1979 and 1989 $\left(M_{L}=5.0\right)$ Malibu [Hauksson and Saldivar, 1986] and 1987 $\left(M_{L}=5.9\right)$ Whittier Narrows [Hauksson and Jones, 1989] sequences. The small earthquakes do not show any simple correlation with late Quaternary faults. As pointed out by Hauksson [1987], a broad zone of activity around the Newport-Inglewood fault can be seen in Figure 2. The absence of epicenters to the north of the southernmost north dipping frontal faults (e.g., the Santa MonicaHollywood and Sierra Madre faults) suggests that the small earthquakes are not associated with these faults. The concentration of small earthquakes to the south of these faults, the zone west of the Whittier fault, and the zone partly offshore to the west of the Newport-Inglewood fault suggests that the deformation is concentrated along the flanks of the basin.

To determine if the 244 earthquakes provide a representative sample over the 2.5-5.9 magnitude range and if their $b$ value depends on the focal mechanisms of the earthquakes, $b$ values have been calculated. The maximum likelihood method [Page, 1968] was used to calculate the $b$ value for the set of events for which focal mechanisms have been determined (Figure 7). The strike-slip and normal faulting events show slightly higher $b$ values of $1.06 \pm 0.17$ and $0.94 \pm 0.39$, respectively, as compared to the $b$ value for thrust faulting events of $0.70 \pm 0.15$. The error bars are the approximate $95 \%$ confidence limits. The average $b$ value for the whole data set is $0.94 \pm 0.12$, similar to the $b$ value of 1.02 determined by Allen et al. [1965] for the Los Angeles basin. This similarity shows that the data set is a representative sample. The strike-slip and normal events thus seem to cause the high $b$ value in the basin as compared to $b$ values in the range 0.8-0.9 for other parts of southern California [Allen et al., 1965]. If Scholz's [1968] correlation of low $b$ values with high stress is correct, the low $b$ value for thrust faults could imply higher stresses on thrust faults as compared with the stresses on strike-slip faults.

\section{Active FaULTING}

The style of active faulting in the Los Angeles basin is reflected in the focal mechanisms of the local earthquakes. The 244 single-event, lower hemisphere focal mechanisms plotted at their epicenters in Figure 8 and listed in Table 1 show that strike-slip, thrust, and normal faulting are all occurring in the basin. The events $(2.5 \leq M \leq 5.9)$ are distributed throughout the Los Angeles basin and the adjacent Santa Monica Bay and San Pedro Bay.

The style of active faulting is also recorded as slip on mappable surficial faults. The major mappable surficial faults in the Los Angeles basin are the northwest striking Newport-Inglewood, Whittier, and Palos Verdes faults and the west striking faults located within and along the southern margin of the central Transverse Ranges, such as the Santa Monica-Hollywood and Sierra Madre faults (Figure 9a). The late Quaternary faults in Figure $9 a$ are from Ziony and Jones [1989] and Ziony and Yerkes [1985], who point out that more than 95 late Quaternary faults have been mapped in the greater Los Angeles area. Many of these faults are capable of generating moderate-sized and large $(M>6.7)$ earthquakes, although some may be too small or not have a favorable orientation to the present stress field for generating large earthquakes. The presence of nearby small earthquakes may assist in evaluating the type of faulting and whether a fault is active, while the absence of small earthquakes near a fault does not exclude the possible future occurrence of small or large earthquakes [Allen et al., 1965; Ziony and Yerkes, 1985].

The surficial folding of sediments above concealed thrust faults is yet another expression of active faulting in the basin. Mapped folds in the basin, represented by their axes in figures, form two major belts of folding. The first fold belt is located along the east and north flank of the Los Angeles basin and the second along the southwest flank of the basin (Figure 9b). Most of the long fold axes in Figure $9 b$ are from Yerkes et al. [1965], who did a comprehensive analysis of the Los Angeles basin geology. Additional fold axes for the offshore area are taken from Junger and Wagner [1977], and Nardin and Henyey [1978]. Harding and Tuminas [1988], and Wright [1987] provided numerous fold axes derived from oil exploration. The Santa Monica Mountains Anticlinorium (SMMA) is shown with a dashed thick curve [Davis et al., 1989]. 
等

(2)

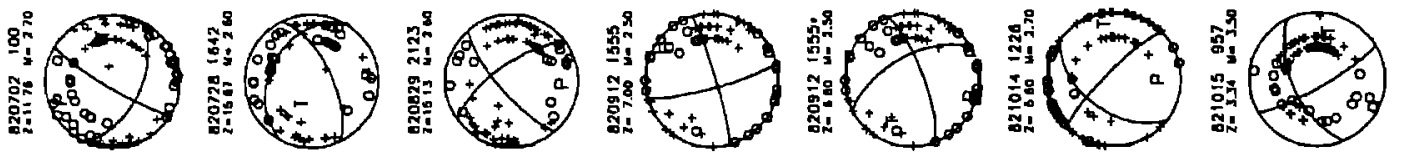

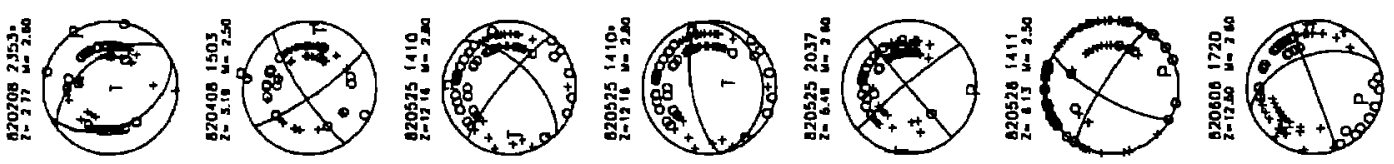

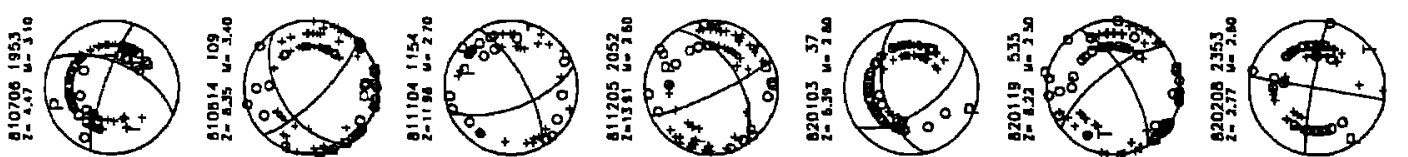

等

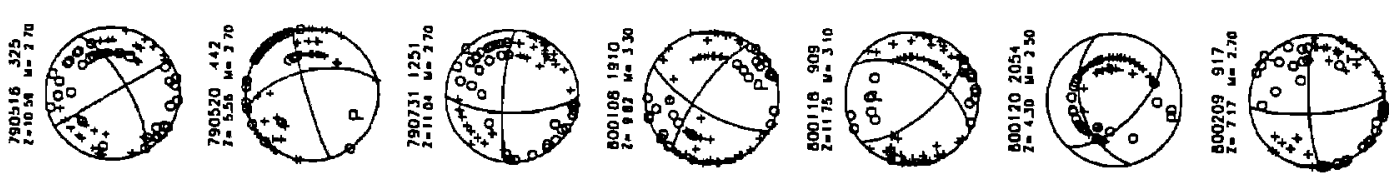

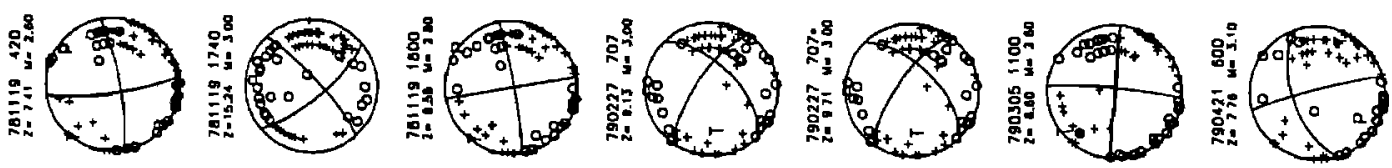

(lot)

(400)

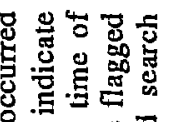

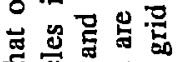

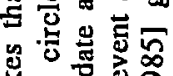

兽实

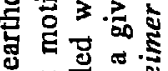

范要点

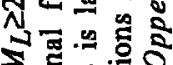

형

虫

宸

突

苛商。

两

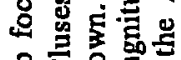

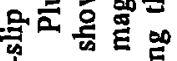

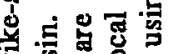

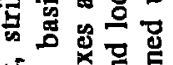

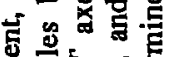

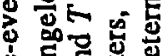

这密

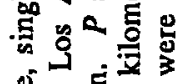

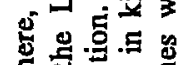

독

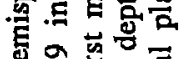

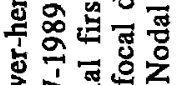

要势

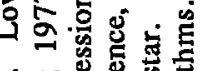

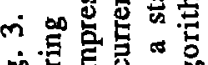

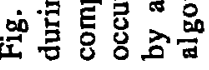

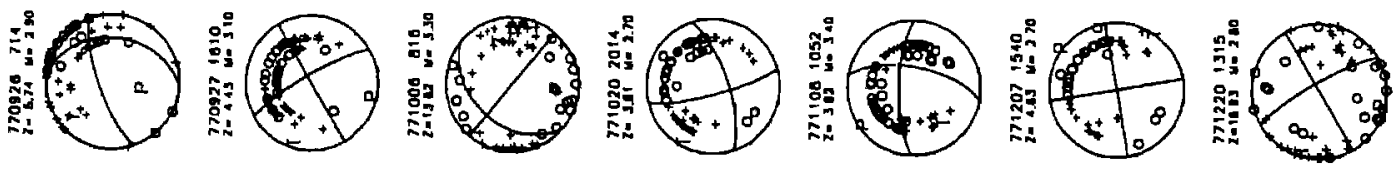

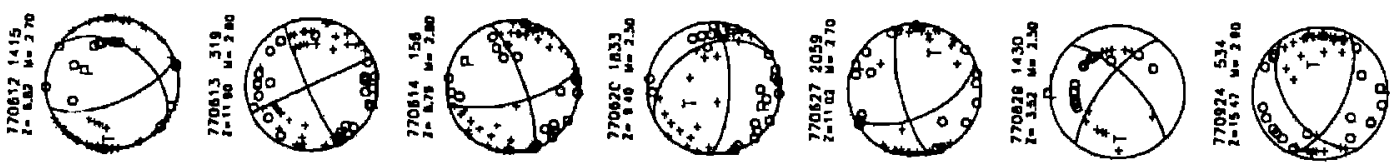




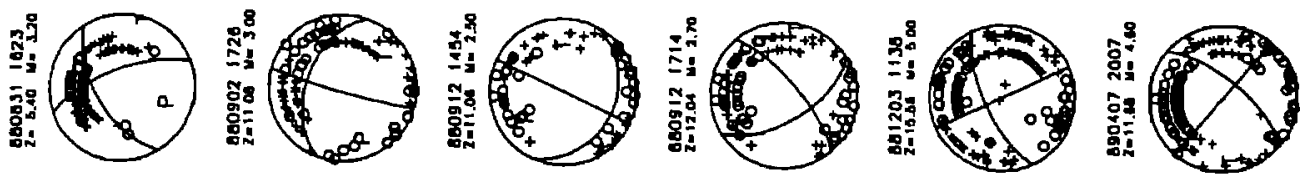

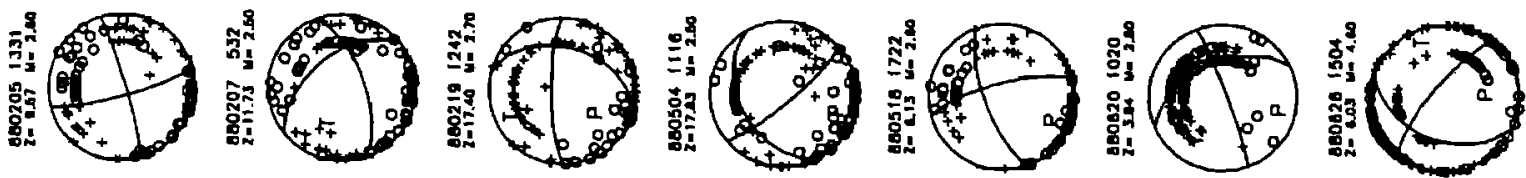

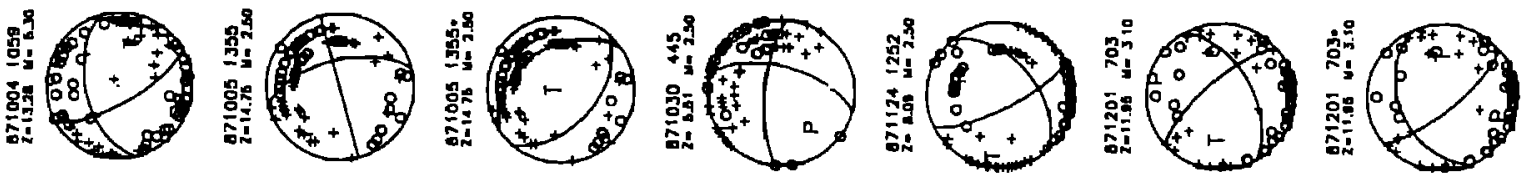

(1)

(140)

(n)

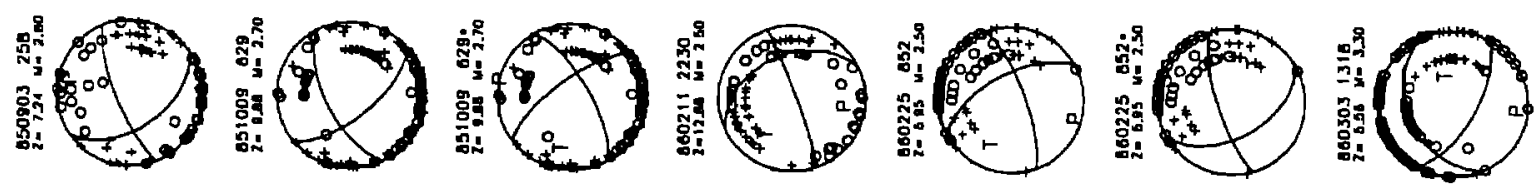

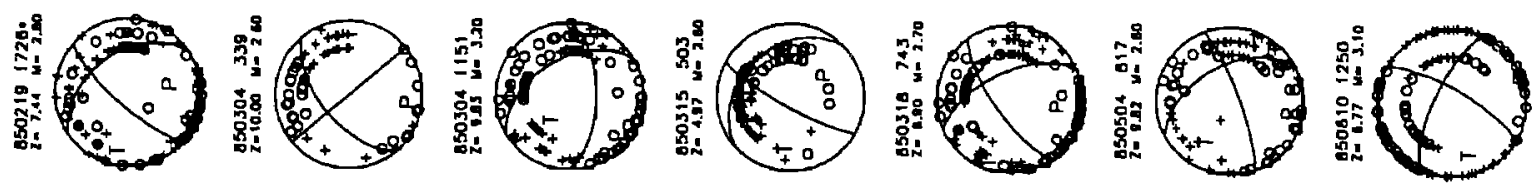

(2)

年

(1) 


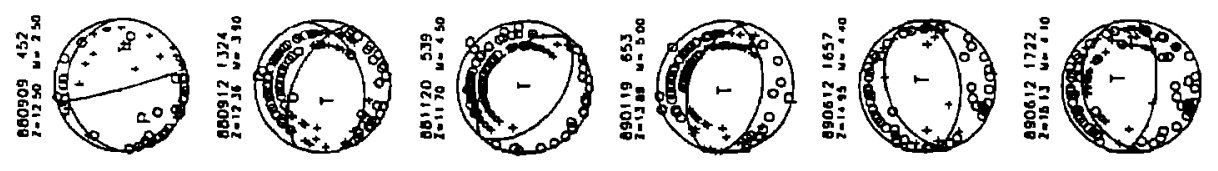

(1-)

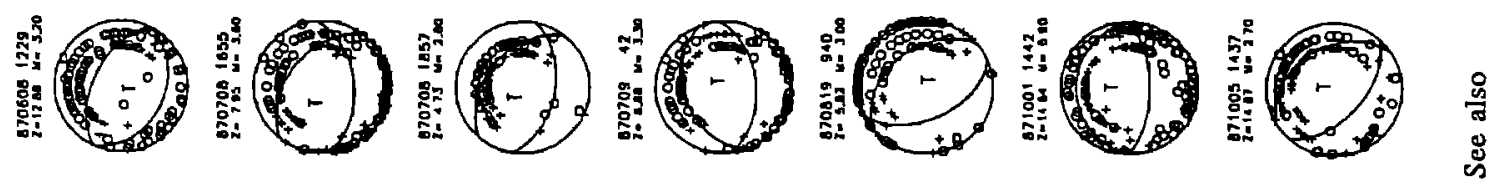

(1)

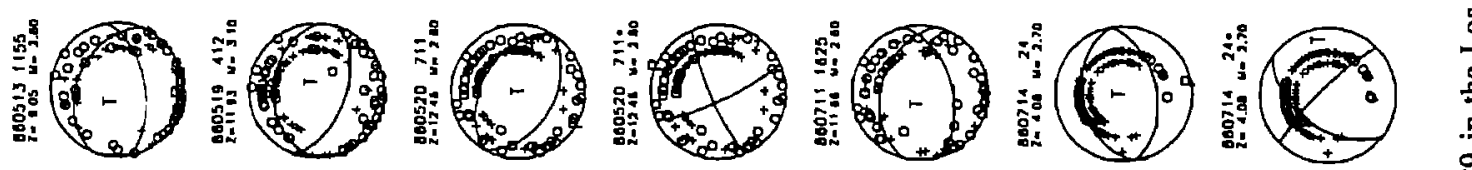

(2)

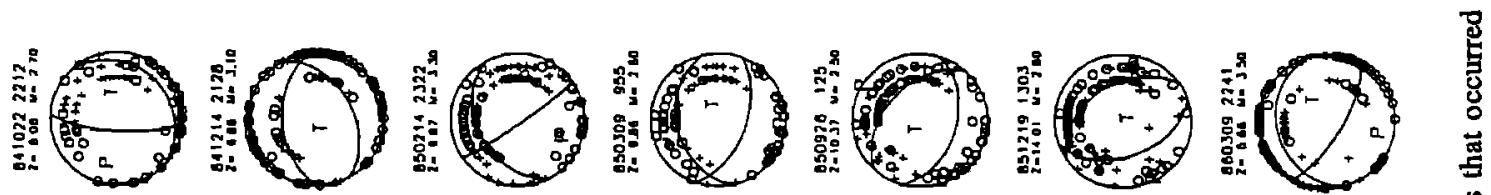

(1)

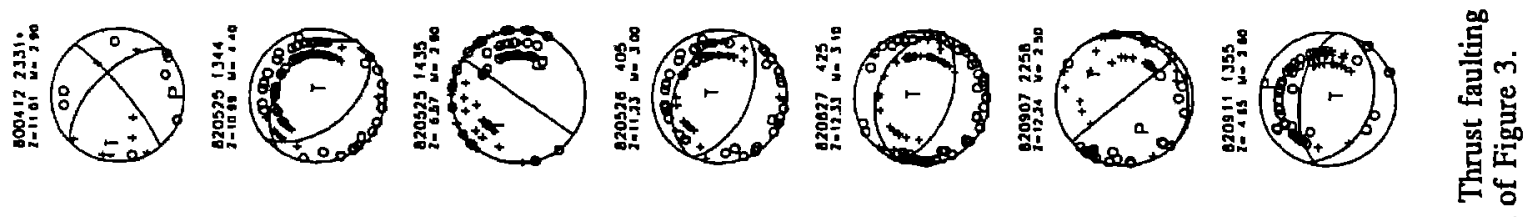

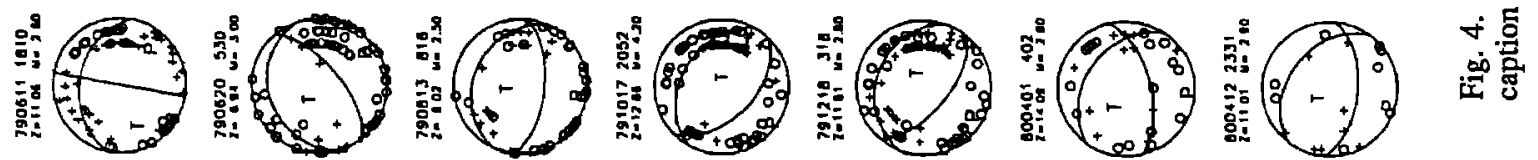

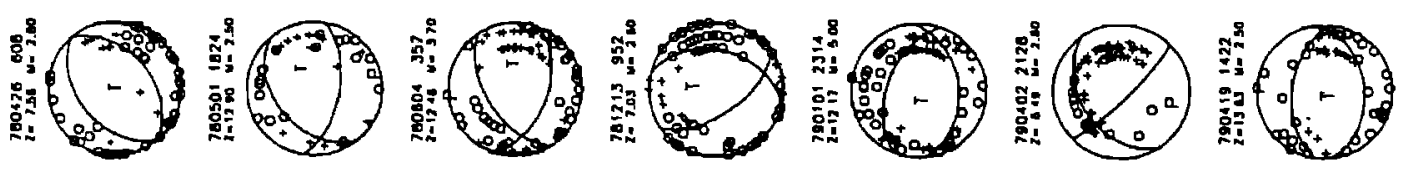

(bid) 


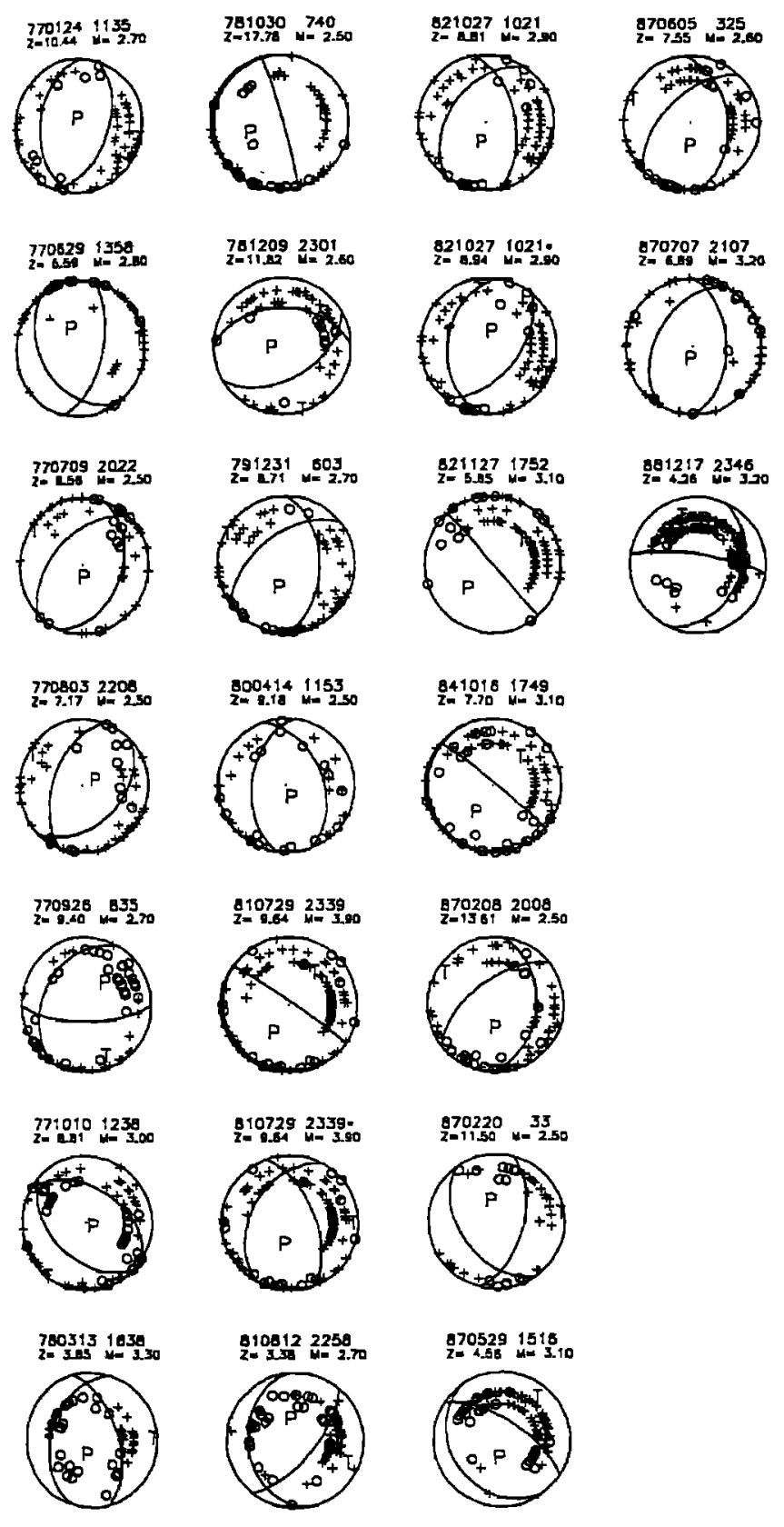

Fig. 5. Normal faulting focal mechanisms that occurred during 1977-1989 in the Los Angeles basin. See also caption of Figure 3.

These folds are assumed to be caused by slip on concealed thrust faults at depth [Suppe, 1985; Davis et al., 1989]. The length of the fold axes gives some indication of possible maximum earthquake size, because the longer the fold axis the larger the concealed fault.

To compare the earthquake focal mechanisms to the late Quatemary faults and folds, the focal mechanisms have been grouped by rake for the selected plane into strike-slip, normal, and thrust faulting. More than half or 144 of these events show strike-slip faulting, 78 show reverse or thrust faulting and 22 show normal faulting. Below, strike-slip mechanisms are compared to mappable surficial strike-slip faults, while thrust and normal mechanisms are compared to zones of folding and thrust faulting.

\section{Strike-Slip Faulting}

To examine strike-slip faulting in the Los Angeles basin, late Quaternary faults and strike-slip focal mechanisms are plotted together in Figure 10. The northeast trending depth cross section AA' in Figure 10 shows that strike-slip faulting occurs from the near surface down to depths of 14-17 km.

The cluster of right-lateral strike-slip mechanisms along the Newport-Inglewood fault can be clearly seen (Figure 10). The largest event $\left(M_{L}=4.6\right)$ on the NewportInglewood fault since the 1940s was strike slip and occurred on April 7, 1989, just 2-3 km to the south of the epicenter of the $1933\left(M_{L}=6.3\right)$ Long Beach earthquake [Richter, 1958]. The Newport-Inglewood fault has more seismic activity associated with it than other late Quaternary faults within the Los Angeles basin.

The spatial correlation between the surficial trace of the Palos Verdes fault and the distribution of strike-slip mechanisms is much less clear than along the NewportInglewood fault. Over more than $60 \mathrm{~km}$ on the Palos Verdes Peninsula and in San Pedro Bay, only four strikeslip mechanisms are located near the surface trace of the fault. In Santa Monica Bay, a small cluster of strike-slip events coincides with the surface trace of the Palos Verdes fault. Several strike-slip events are located farther west in Santa Monica Bay, along the western edge of the Santa Monica Shelf Projection [Hauksson and Saldivar, 1989]. Other offshore strike-slip events cannot be associated with specific mapped faults.

In the eastern Los Angeles basin the strike-slip focal mechanisms do not cluster around either the Whittier or the Chino fault (Figure 10). A northwest trending distribution of strike-slip mechanisms $5-10 \mathrm{~km}$ west of the Whittier fault is too scattered to correlate with one specific fault such as the Norwalk fault. An alignment of left-lateral focal mechanisms trending northeast from Yorba Linda (Yorba Linda trend) and crossing the Whittier fault, however, suggests a northeast trending left-lateral strikeslip fault (Figures 2 and 10). Less likely, these could be interpreted to be an en echelon pattem of north-northwest striking faults, which form an apparent northeast trend. This trend of epicenters and focal mechanisms that has not been pointed out before in the literature crosses the fold belt along the eastem flank of the basin. It may also be associated with a cluster of strike-slip events located farther to the northeast, $8-16 \mathrm{~km}$ east of the Chino fault. This trend may also segment or offset concealed thrust faults at depth because the focal depths of some of these strike-slip events are greater than the depths of the thrust events. Similar northeast trending alignments adjacent to the southern San Andreas fault have been identified by Nicholson et al. [1986].

Several recent Los Angeles basin earthquakes have shown mostly strike-slip movement. The largest strikeslip event to occur during the time period covered by this study was the $1987\left(M_{L}=5.3\right)$ Whittier Narrows aftershock. It occurred on a north-northwest striking tear fault that appeared to limit the western extent of rupture in the mainshock [Hauksson and Jones, 1989]. This steeply dipping strike-slip fault may segment the mainshock thrust fault at depth. A similar north-northwest trending fault is defined by a cluster of strike-slip events located $10 \mathrm{~km}$ farther west near Montebello (Figure 10). The 1988 
TABLE 1. Locations and Focal Mechanisms of Earthquakes in the Los Angeles Basin

\begin{tabular}{|c|c|c|c|c|c|c|c|c|c|}
\hline \multirow{2}{*}{$\begin{array}{l}\text { Origin } \\
\text { Day }\end{array}$} & \multirow{2}{*}{$\begin{array}{l}\text { Time, } \\
\text { Ur }\end{array}$} & \multirow{2}{*}{$\begin{array}{l}\text { Latitude } \\
\mathrm{N}\end{array}$} & \multirow{2}{*}{$\begin{array}{l}\text { Longirude } \\
\mathrm{W}\end{array}$} & \multirow{2}{*}{$\begin{array}{l}\text { Depth, } \\
\text { km }\end{array}$} & \multirow{2}{*}{$\begin{array}{l}\text { Mag. } \\
M_{L}\end{array}$} & \multicolumn{3}{|c|}{ Focal Mechanisms } & Number of \\
\hline & & & & & & Ddir & Dip & Rake & First Motions \\
\hline & & & ke-Slip Faulsi & Earthqu & ak & & & & \\
\hline June 12,1977 & 1415 & $34^{\circ} 01.67^{\circ}$ & $117^{\circ} 34.99^{\prime}$ & 6.8 & 2.7 & $125^{\circ}$ & $40^{\circ}$ & $-40^{\circ}$ & 68 \\
\hline June 13,1977 & 0319 & $33^{\circ} 52.37^{\prime}$ & $118^{\circ} 37.33^{\prime}$ & 11.9 & 2.8 & $245^{\circ}$ & $90^{\circ}$ & $175^{\circ}$ & 66 \\
\hline June 14,1977 & 0156 & $34^{\circ} 00.62^{\prime}$ & $118^{\circ} 19.52^{\prime}$ & 8.7 & 2.8 & $255^{\circ}$ & $70^{\circ}$ & $-164^{\circ}$ & 57 \\
\hline June 20,1977 & 1833 & $34^{\circ} 00.66^{\prime}$ & $118^{\circ} 18.80^{\prime}$ & 9.4 & 2.5 & $59^{\circ}$ & $35^{\circ}$ & $143^{\circ}$ & 48 \\
\hline June 27,1977 & 2059 & $34^{\circ} 05.93^{\prime}$ & $17^{\circ} 57.85^{\prime}$ & 11.0 & 2.7 & $346^{\circ}$ & $73^{\circ}$ & $36^{\circ}$ & 47 \\
\hline Aug. 29, 1977 & 1430 & $33^{\circ} 48.80^{\prime}$ & $117^{\circ} 51.44^{\prime}$ & 3.5 & 2.5 & $37^{\circ}$ & $71^{\circ}$ & $158^{\circ}$ & 29 \\
\hline Sept. 24,1977 & 0534 & $34^{\circ} 03.94^{\prime}$ & $117^{\circ} 58.04^{\prime}$ & 15.4 & 2.9 & $325^{\circ}$ & $48^{\circ}$ & $41^{\circ}$ & 51 \\
\hline Sept. 26,1977 & 0714 & $33^{\circ} 32.29^{\prime}$ & $118^{\circ} 13.58^{\prime}$ & 5.7 & 2.9 & $95^{\circ}$ & $35^{\circ}$ & $-150^{\circ}$ & 57 \\
\hline Sept. 27,1977 & 1810 & $33^{\circ} 32.17^{\prime}$ & $118^{\circ} 12.97^{\prime}$ & 4.4 & 3.1 & $60^{\circ}$ & $80^{\circ}$ & $180^{\circ}$ & 60 \\
\hline Oct. 6,1977 & 0816 & $33^{\circ} 59.96^{\circ}$ & $118^{\circ} 11.69^{\prime}$ & 13.6 & 3.3 & $310^{\circ}$ & $40^{\circ}$ & $0^{\circ}$ & 57 \\
\hline Oet. 20.1977 & 2014 & $33^{\circ} 44.31^{\prime}$ & $118^{\circ} 01.02^{\prime}$ & 3.8 & 2.7 & $255^{\circ}$ & $80^{\circ}$ & $-160^{\circ}$ & 54 \\
\hline Nov. 8,1977 & 1052 & $33^{\circ} 53.54^{\prime}$ & $117^{\circ} 54.68$ & 3.9 & 3.4 & $100^{\circ}$ & $65^{\circ}$ & $20^{\circ}$ & 65 \\
\hline Dec. 7,1977 & 1540 & $33^{\circ} 47.32^{\prime}$ & $118^{\circ} 04.78^{\prime}$ & 4.6 & 2.7 & $170^{\circ}$ & $90^{\circ}$ & $0^{\circ}$ & 48 \\
\hline Dec. 20, 1977 & 1315 & $34^{\circ} 00.98^{\prime}$ & $118^{\circ} 12.00^{\prime}$ & 16.9 & 2.8 & $60^{\circ}$ & $80^{\circ}$ & $180^{\circ}$ & 51 \\
\hline Feb. 12, 1978 & 1522 & $33^{\circ} 57.52^{\circ}$ & $117^{\circ} 43.06^{\prime}$ & 4.8 & 2.7 & $15^{\circ}$ & $80^{\circ}$ & $154^{\circ}$ & 46 \\
\hline April 18,1978 & 2242 & $33^{\circ} 53.16^{\circ}$ & $117^{\circ} 32.53^{\prime}$ & 8.9 & 3.1 & $122^{\circ}$ & $56^{\circ}$ & $-37^{\circ}$ & 56 \\
\hline April 29,1978 & 0332 & $33^{\circ} 50.42^{\prime}$ & $117^{\circ} 43.58^{\prime}$ & 9.5 & 3.3 & $321^{\circ}$ & $38^{\circ}$ & $43^{\circ}$ & 79 \\
\hline May 9,1978 & 1830 & $33^{\circ} 48.11^{\prime}$ & $117^{\circ} 58.41^{\prime}$ & 14.0 & 2.7 & $250^{\circ}$ & $60^{\circ}$ & $-168^{\circ}$ & 58 \\
\hline May 11,1978 & 0547 & $33^{\circ} 59.48^{\prime}$ & $118^{\circ} 21.36^{\prime}$ & 7.7 & 2.9 & $259^{\circ}$ & $60^{\circ}$ & $168^{\circ}$ & 65 \\
\hline May 11,1978 & 1757 & $34^{\circ} 00.76^{\prime}$ & $118^{\circ} 28.03^{\prime}$ & 8.4 & 2.8 & $236^{\circ}$ & $80^{\circ}$ & $159^{\circ}$ & 58 \\
\hline May 27,1978 & 1438 & $34^{\circ} 00.79^{\prime}$ & $117^{\circ} 36.06^{\prime}$ & 4.5 & 2.5 & $245^{\circ}$ & $85^{\circ}$ & $150^{\circ}$ & 59 \\
\hline June 14,1978 & 0559 & $34^{\circ} 00.88^{\circ}$ & $117^{\circ} 36.07^{\prime}$ & 5.0 & 2.5 & $51^{\circ}$ & $80^{\circ}$ & $159^{\circ}$ & 55 \\
\hline Sept. 2, 1978 & 1815 & $33^{\circ} 57.22^{\prime}$ & $117^{\circ} 43.06^{\prime}$ & 4.7 & 2.7 & $195^{\circ}$ & $80^{\circ}$ & $170^{\circ}$ & 68 \\
\hline Sept 3,1978 & 1810 & $33^{\circ} 57.27^{\prime}$ & $117^{\circ} 43.32^{\prime}$ & 5.6 & 3.5 & $306^{\circ}$ & $64^{\circ}$ & $33^{\circ}$ & 100 \\
\hline Sept. 22, 1978 & 0313 & $33^{\circ} 52.07^{\prime}$ & $117^{\circ} 49.67^{\prime}$ & 1.9 & 2.7 & $290^{\circ}$ & $80^{\circ}$ & $-10^{\circ}$ & 60 \\
\hline Oct. 6,1978 & 1339 & $33^{\circ} 55.07^{\prime}$ & $117^{\circ} 45.55^{\prime}$ & 14.4 & 2.5 & $315^{\circ}$ & $70^{\circ}$ & $-30^{\circ}$ & 67 \\
\hline Nov. 19,1978 & 0355 & $34^{\circ} 00.78^{\prime}$ & $118^{\circ} 37.69^{\prime}$ & 11.8 & 2.8 & $64^{\circ}$ & $24^{\circ}$ & $141^{\circ}$ & 76 \\
\hline Nov. 19,1978 & 0420 & $33^{\circ} 51.05^{\prime}$ & $118^{\circ} 10.17^{\prime}$ & 7.4 & 2.6 & $170^{\circ}$ & $80^{\circ}$ & $-10^{\circ}$ & 60 \\
\hline Nov. 19,1978 & 1740 & $33^{\circ} 50.61^{\prime}$ & $118^{\circ} 10.23^{\prime}$ & 15.2 & 3.0 & $230^{\circ}$ & $75^{\circ}$ & $-170^{\circ}$ & 71 \\
\hline Nov. 19,1978 & 1800 & $33^{\circ} 50.97^{\prime}$ & $118^{\circ} 09.93^{\prime}$ & 8.5 & 2.8 & $170^{\circ}$ & $85^{\circ}$ & $0^{\circ}$ & 66 \\
\hline Feb. 27, 1979 & 0707 & $33^{\circ} 56.71^{\prime}$ & $118^{\circ} 19.50^{\prime}$ & 9.1 & 3.0 & $34^{\circ}$ & $72^{\circ}$ & $148^{\circ}$ & 66 \\
\hline March 5, 1979 & 1100 & $33^{\circ} 47.96^{\circ}$ & $118^{\circ} 07.80^{\circ}$ & 8.6 & 2.6 & $95^{\circ}$ & $85^{\circ}$ & $-180^{\circ}$ & 60 \\
\hline April 21, 1979 & 0600 & $33^{\circ} 47.64^{\prime}$ & $118^{\circ} 05.02^{\circ}$ & 7.7 & 3.1 & $75^{\circ}$ & $85^{\circ}$ & $-150^{\circ}$ & 64 \\
\hline May 16,1979 & 0325 & $33^{\circ} 51.00^{\prime}$ & $118^{\circ} 31.31^{\prime}$ & 10.5 & 2.7 & $60^{\circ}$ & $90^{\circ}$ & $160^{\circ}$ & 77 \\
\hline May 20, 1979 & 0442 & $33^{\circ} 51.17^{\prime}$ & $118^{\circ} 31.46^{\prime}$ & 5.5 & 2.7 & $77^{\circ}$ & $60^{\circ}$ & $-174^{\circ}$ & 75 \\
\hline July 31, 1979 & 1251 & $33^{\circ} 50.52^{\prime}$ & $118^{\circ} 07.81^{\prime}$ & 11.0 & 2.7 & $270^{\circ}$ & $70^{\circ}$ & $170^{\circ}$ & 69 \\
\hline Jan. 8,1980 & 1910 & $34^{\circ} 02.31^{\prime}$ & $117^{\circ} 33.26^{\prime}$ & 9.8 & 3.3 & $35^{\circ}$ & $75^{\circ}$ & $-160^{\circ}$ & 92 \\
\hline Jan. 18,1980 & 0909 & $33^{\circ} 55.95^{\prime}$ & $117^{\circ} 43.25^{\circ}$ & 11.7 & 3.1 & $120^{\circ}$ & $54^{\circ}$ & $-31^{\circ}$ & 80 \\
\hline $\operatorname{Jan} .20,1980$ & 2054 & $33^{\circ} 45.97^{\circ}$ & $117^{\circ} 31.86^{\circ}$ & 4.3 & 2.5 & $345^{\circ}$ & $48^{\circ}$ & $41^{\circ}$ & 65 \\
\hline Feb. 9,1980 & 0917 & $33^{\circ} 47.87^{\prime}$ & $118^{\circ} 06.17^{\circ}$ & 7.1 & 2.7 & $265^{\circ}$ & $75^{\circ}$ & $170^{\circ}$ & 63 \\
\hline April 21,1980 & 2255 & $34^{\circ} 09.21^{\prime}$ & $117^{\circ} 47.28^{\prime}$ & 11.7 & 2.7 & $330^{\circ}$ & $75^{\circ}$ & $-30^{\circ}$ & 70 \\
\hline Feb. 27, 1981 & 1511 & $34^{\circ} 09.37^{\prime}$ & $118^{\circ} 35.66^{\prime}$ & 14.3 & 3.5 & $255^{\circ}$ & $75^{\circ}$ & $-180^{\circ}$ & 84 \\
\hline May 6,1981 & 0556 & $33^{\circ} 44.05^{\prime}$ & $118^{\circ} 01.60^{\prime}$ & 3.7 & 3.1 & $90^{\circ}$ & $55^{\circ}$ & $-140^{\circ}$ & 78 \\
\hline June 4, 1981 & 1151 & $33^{\circ} 40.81^{\prime}$ & $117^{\circ} 22.39^{\circ}$ & 11.7 & 3.6 & $215^{\circ}$ & $80^{\circ}$ & $150^{\circ}$ & 80 \\
\hline June 4, 1981 & 1426 & $33^{\circ} 40.74^{\prime}$ & $117^{\circ} 22.47^{\circ}$ & 12.0 & 3.0 & $38^{\circ}$ & $52^{\circ}$ & $154^{\circ}$ & 52 \\
\hline June 22, 1981 & 1803 & $33^{\circ} 44.92^{\prime}$ & $118^{\circ} 06.55^{\circ}$ & 2.9 & 2.5 & $10^{\circ}$ & $90^{\circ}$ & $-10^{\circ}$ & 72 \\
\hline July 5,1981 & 0031 & $33^{\circ} 40.86^{\prime}$ & $117^{\circ} 22.43^{\prime}$ & 4.1 & 2.8 & $35^{\circ}$ & $90^{\circ}$ & $175^{\circ}$ & 77 \\
\hline July 6,1981 & 1953 & $33^{\circ} 51.72^{\prime}$ & $117^{\circ} 51.90^{\circ}$ & 4.4 & 3.1 & $19^{\circ}$ & $81^{\circ}$ & $149^{\circ}$ & 81 \\
\hline Aug. 14, 1981 & 0109 & $33^{\circ} 57.79^{\prime}$ & $118^{\circ} 34.32^{\prime}$ & 83 & 3.4 & $225^{\circ}$ & $75^{\circ}$ & $140^{\circ}$ & 94 \\
\hline Nov. 4,1981 & 1154 & $33^{\circ} 54.46^{\prime}$ & $118^{\circ} 37.04^{\prime}$ & 11.9 & 2.7 & $250^{\circ}$ & $65^{\circ}$ & $-160^{\circ}$ & 48 \\
\hline Dec. 5,1981 & 2052 & $34^{\circ} 05.46^{\prime}$ & $117^{\circ} 47.59^{\prime}$ & 13.9 & 2.5 & $245^{\circ}$ & $81^{\circ}$ & $-144^{\circ}$ & 77 \\
\hline Jan. 3,1982 & 0037 & $33^{\circ} 54.72^{\prime}$ & $117^{\circ} 57.32^{\circ}$ & 5.3 & 2.8 & $347^{\circ}$ & $56^{\circ}$ & $37^{\circ}$ & 87 \\
\hline Jan. 19,1982 & 0535 & $33^{\circ} 55.97^{\circ}$ & $118^{\circ} 28.75^{\circ}$ & 8.2 & 2.5 & $52^{\circ}$ & $71^{\circ}$ & $158^{\circ}$ & 98 \\
\hline Feb. 8, 1982 & 2353 & $34^{\circ} 14.82^{\circ}$ & $118^{\circ} 24.56^{\prime}$ & 2.7 & 2.6 & $280^{\circ}$ & $90^{\circ}$ & $175^{\circ}$ & 67 \\
\hline April 8,1982 & 1503 & $34^{\circ} 11.82^{\prime}$ & $118^{\circ} 38.66^{\circ}$ & 3.1 & 2.5 & $235^{\circ}$ & $80^{\circ}$ & $170^{\circ}$ & 65 \\
\hline May 25, 1982 & 1410 & $33^{\circ} 33.04^{\prime}$ & $118^{\circ} 12.03^{\circ}$ & 12.1 & 2.8 & $39^{\circ}$ & $81^{\circ}$ & $149^{\circ}$ & 78 \\
\hline May 25, 1982 & 2037 & $33^{\circ} 33.22^{\prime}$ & $118^{\circ} 11.83^{\circ}$ & 5.4 & 2.6 & $50^{\circ}$ & $85^{\circ}$ & $180^{\circ}$ & 76 \\
\hline May 26,1982 & 1411 & $33^{\circ} 38.40^{\prime \prime}$ & $117^{\circ} 29.02^{\prime}$ & 6.1 & 2.5 & $32^{\circ}$ & $80^{\circ}$ & $-164^{\circ}$ & 84 \\
\hline Iune 6,1982 & 1720 & $33^{\circ} 28.89^{\prime}$ & $117^{\circ} 43.91^{\prime}$ & 12.5 & 2.6 & $70^{\circ}$ & $50^{\circ}$ & $180^{\circ}$ & 64 \\
\hline July 2,1982 & 0100 & $33^{\circ} 49.24^{\prime}$ & $117^{\circ} 43.59^{\prime}$ & 11.7 & 2.7 & $205^{\circ}$ & $55^{\circ}$ & $170^{\circ}$ & 87 \\
\hline July 28,1982 & 1642 & $34^{\circ} 14.31^{\prime}$ & $118^{\circ} 33.10^{\circ}$ & 15.6 & 2.6 & $28^{\circ}$ & $38^{\circ}$ & $137^{\circ}$ & 63 \\
\hline Aug. 29, 1982 & 2123 & $34^{\circ} 03.48^{\prime}$ & $117^{\circ} 35.96^{\prime}$ & 15.1 & 2.6 & $55^{\circ}$ & $70^{\circ}$ & $-164^{\circ}$ & 73 \\
\hline Sept. 12,1982 & 1555 & $33^{\circ} 48.18^{\prime}$ & $118^{\circ} 14.10^{\prime}$ & 7.0 & 2.5 & $255^{\circ}$ & $80^{\circ}$ & $-174^{\circ}$ & 67 \\
\hline Oet. 14,1982 & 1226 & $33^{\circ} 53.18^{\prime}$ & $117^{\circ} 41.09^{\prime}$ & 5.8 & 2.7 & $45^{c}$ & $80^{\circ}$ & $-140^{\circ}$ & 77 \\
\hline Oct. 15,1982 & 0957 & $34^{\circ} 11.88^{\prime}$ & $118^{\circ} 38.73^{\prime}$ & 3.3 & 3.5 & $240^{\circ}$ & $80^{\circ}$ & $150^{\circ}$ & 100 \\
\hline Nov, 4, 1982 & 1613 & $33^{\circ} 53.27^{\circ}$ & $117^{\circ} 54.82^{\prime}$ & 12.9 & 2.6 & $316^{\circ}$ & $41^{\circ}$ & $15^{\circ}$ & 75 \\
\hline Nov. 6,1982 & 0737 & $33^{\circ} 48.12^{\prime}$ & $118^{\circ} 13.92^{\prime}$ & 8.2 & 2.8 & $90^{\circ}$ & $75^{\circ}$ & $-170^{\circ}$ & 77 \\
\hline Dec. 13,1982 & 0205 & $33^{\circ} 42.50^{\prime}$ & $118^{\circ} 04.68^{\prime}$ & 8.4 & 2.6 & $85^{\circ}$ & $90^{\circ}$ & $-170^{\circ}$ & 51 \\
\hline $\operatorname{Jan} .21,1983$ & 1450 & $34^{\circ} 09.19^{\prime}$ & $117^{\circ} 48.69^{\prime}$ & 12.1 & 2.5 & $323^{\circ}$ & $38^{\circ}$ & $-43^{\circ}$ & 65 \\
\hline March 1, 1983 & 2018 & $33^{\circ} 55.95^{\prime}$ & $118^{\circ} 17.40^{\prime}$ & 10.8 & 3.6 & $250^{\circ}$ & $80^{\circ}$ & $160^{\circ}$ & 64 \\
\hline April 18,1983 & 2139 & $34^{\circ} 01.88^{\prime}$ & $118^{\circ} 12.88^{\prime}$ & 13.1 & 2.8 & $1^{\circ}$ & $38^{\circ}$ & $43^{\circ}$ & 66 \\
\hline July 13,1983 & 2353 & $33^{\circ} 41.37^{\prime}$ & $118^{\circ} 01.68^{\prime}$ & 5.4 & 2.5 & $261^{\circ}$ & $80^{\circ}$ & $-169^{\circ}$ & 41 \\
\hline
\end{tabular}


TABLE 1. (continued)

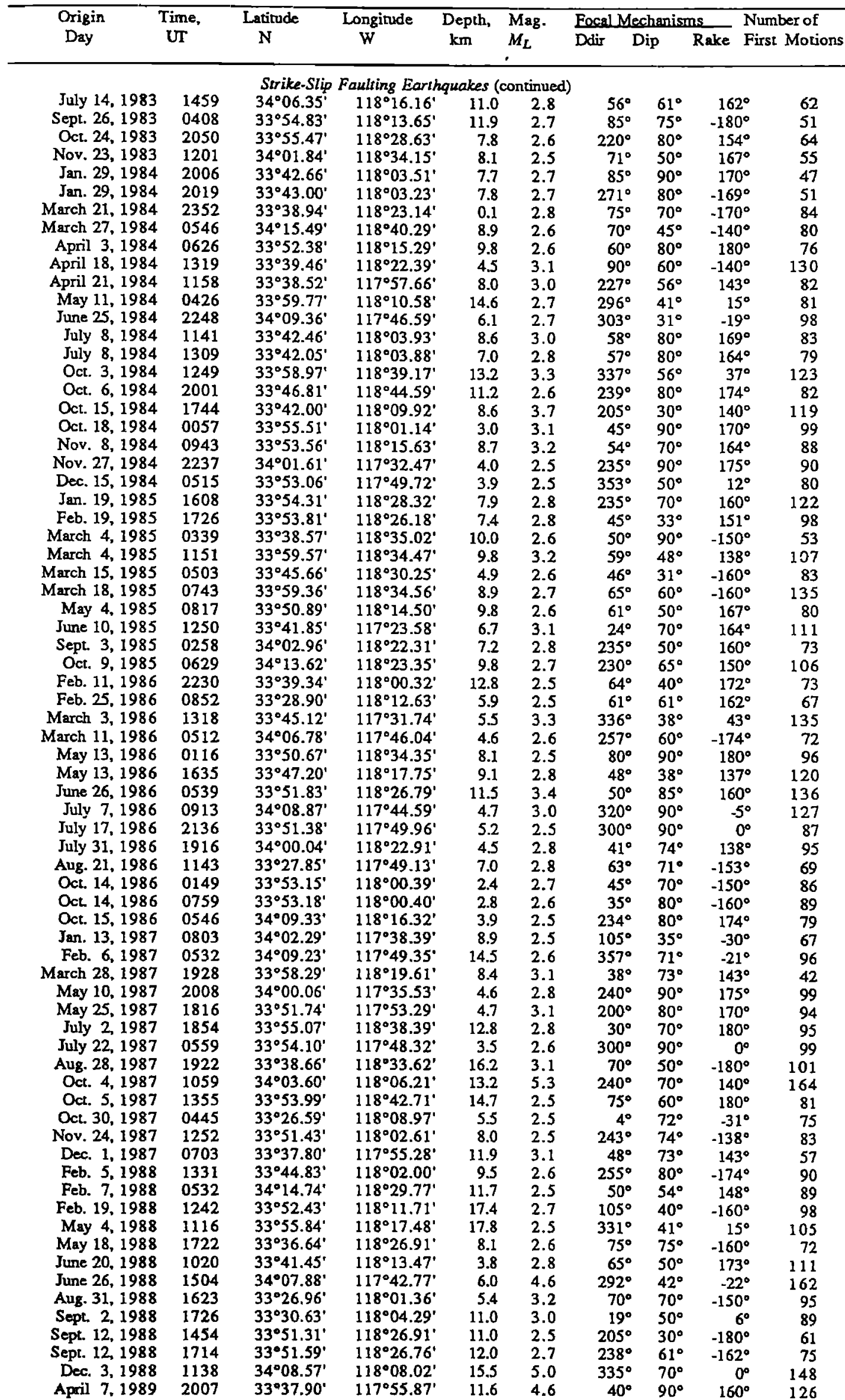


TABLE 1. (continued)

\begin{tabular}{|c|c|c|c|c|c|c|c|c|c|}
\hline \multirow{2}{*}{$\begin{array}{l}\text { Origin } \\
\text { Day }\end{array}$} & \multirow{2}{*}{$\begin{array}{l}\text { Time, } \\
\text { UT }\end{array}$} & \multirow{2}{*}{$\begin{array}{l}\text { Latinude } \\
\mathrm{N}\end{array}$} & \multirow{2}{*}{$\begin{array}{l}\text { Longitude } \\
\mathrm{W}\end{array}$} & \multirow{2}{*}{$\begin{array}{l}\text { Depth, } \\
\text { km }\end{array}$} & \multirow{2}{*}{$\begin{array}{l}\text { Mag. } \\
M_{L}\end{array}$} & \multicolumn{3}{|c|}{ Focal Mechanisms } & \multirow{2}{*}{$\begin{array}{l}\text { Number of } \\
\text { First Mot }\end{array}$} \\
\hline & & & & & & Ddir & Dip & Rake & \\
\hline & & & Thrust Faulting & arthque & akes & & & & \\
\hline Sept. 14,1977 & 2135 & $33^{\circ} 52.82^{\prime}$ & $117^{\circ} 49.51^{\circ}$ & 2.0 & 2.9 & $357^{\circ}$ & $61^{\circ}$ & $78^{\circ}$ & 49 \\
\hline Oet. 16,1977 & 1702 & $33^{\circ} 45.14^{\prime}$ & $117^{\circ} 42.51^{\prime}$ & 7.6 & 2.6 & $200^{\circ}$ & $5^{\circ}$ & $170^{\circ}$ & 35 \\
\hline Oct. 24,1977 & 1936 & $33^{\circ} 32.98^{\prime}$ & $118^{\circ} 14.53^{\prime}$ & 5.6 & 2.8 & $255^{\circ}$ & $65^{\circ}$ & $110^{\circ}$ & 36 \\
\hline Dec. 27,1977 & 0607 & $33^{\circ} 54.31^{\prime}$ & $118^{\circ} 32.80^{\prime}$ & 8.9 & 2.7 & $14^{\circ}$ & $51^{\circ}$ & $47^{\circ}$ & 75 \\
\hline March 8,1978 & 1449 & $33^{\circ} 49.34^{\prime}$ & $117^{\circ} 53.43^{\prime}$ & 5.7 & 3.0 & $52^{\circ}$ & $39^{\circ}$ & $63^{\circ}$ & 82 \\
\hline March 14,1978 & 2359 & $34^{\circ} 00.07^{\prime}$ & $118^{\circ} 40.44^{\prime}$ & 13.6 & 3.1 & $3^{\circ}$ & $35^{\circ}$ & $59^{\circ}$ & 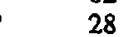 \\
\hline April 26, 1978 & 0608 & $33^{\circ} 56.29^{\prime}$ & $118^{\circ} 20.49^{\prime}$ & 75 & 2.8 & $320^{\circ}$ & $45^{\circ}$ & $90^{\circ}$ & 59 \\
\hline May 1,1978 & 1824 & $33^{\circ} 56.84^{\prime}$ & $118^{\circ} 44.20^{\circ}$ & 12.9 & 2.5 & $320^{\circ}$ & $41^{\circ}$ & $49^{\circ}$ & 37 \\
\hline June 4,1978 & 0357 & $33^{\circ} 55.21^{\prime}$ & $117^{\circ} 50.32^{\prime}$ & 12.4 & 3.7 & $327^{\circ}$ & $54^{\circ}$ & $52^{\circ}$ & 81 \\
\hline Dec. 13,1978 & 0952 & $33^{\circ} 35.44^{\prime}$ & $118^{\circ} 02.04^{\prime}$ & 7.0 & 2.6 & $235^{\circ}$ & $60^{\circ}$ & $60^{\circ}$ & 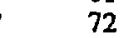 \\
\hline Jan. 1,1979 & 2314 & $33^{\circ} 57.05^{\prime}$ & $118^{\circ} 40.35^{\prime}$ & 12.1 & 5.0 & $20^{\circ}$ & $52^{\circ}$ & $106^{\circ}$ & 80 \\
\hline April 2, 1979 & 2128 & $33^{\circ} 41.92^{\prime}$ & $118^{\circ} 11.78^{\prime}$ & 5.4 & 2.6 & $225^{\circ}$ & $80^{\circ}$ & $130^{\circ}$ & 67 \\
\hline April 19,1979 & 1422 & $34^{\circ} 00.72^{\prime}$ & $118^{\circ} 10.37^{\circ}$ & 13.6 & 2.5 & $7^{\circ}$ & $50^{\circ}$ & $98^{\circ}$ & 53 \\
\hline June 11, 1979 & 1810 & $33^{\circ} 50.38^{\prime}$ & $118^{\circ} 32.35^{\prime}$ & 11.0 & 2.6 & $100^{\circ}$ & $90^{\circ}$ & $50^{\circ}$ & 67 \\
\hline June 20,1979 & 0530 & $33^{\circ} 59.62^{\prime}$ & $118^{\circ} 21.23^{\prime}$ & 6.9 & 3.0 & $342^{\circ}$ & $26^{\circ}$ & $110^{\circ}$ & 69 \\
\hline 1979 & 0816 & $33^{\circ} 54.83^{\circ}$ & $118^{\circ} 29.82^{\prime}$ & 8.0 & 2.5 & $24^{\circ}$ & $31^{\circ}$ & $106^{\circ}$ & 52 \\
\hline 7,1979 & 2052 & $33^{\circ} 55.84^{\prime}$ & $118^{\circ} 39.25^{\prime}$ & 12.8 & 4.2 & $29^{\circ}$ & $41^{\circ}$ & $78^{\circ}$ & 94 \\
\hline 1979 & 0318 & 6.68' & $118^{\circ} 39^{3} 39^{\prime}$ & 11.8 & 2.8 & $20^{\circ}$ & $31^{\circ}$ & $73^{\circ}$ & 89 \\
\hline 1980 & 0402 & $34^{\circ} 00.52^{\prime}$ & $118^{\circ} 39.74^{\prime \prime}$ & 14.0 & 2.8 & $34^{\circ}$ & $41^{\circ}$ & $130^{\circ}$ & \\
\hline April 12, 1980 & 2331 & $34^{\circ} 03.12^{\prime}$ & $118^{\circ} 43.03^{\circ}$ & 11.0 & 2.9 & $37^{\circ}$ & $39^{\circ}$ & $116^{\circ}$ & 16 \\
\hline May 25, I982 & 1344 & $33^{\circ} 33.44^{\prime}$ & $118^{\circ} 12.21^{\circ}$ & 10.9 & 4.4 & $230^{\circ}$ & $50^{\circ}$ & $100^{\circ}$ & 121 \\
\hline 1982 & 1435 & $33^{\circ} 32.41^{\prime}$ & $118^{\circ} 11.83^{\prime}$ & 5.5 & 2.9 & $125^{\circ}$ & $90^{\circ}$ & $90^{\circ}$ & 81 \\
\hline 1982 & 0405 & $33^{\circ} 33.40^{\prime}$ & $118^{\circ} 12.22^{\prime}$ & 11.2 & 3.0 & $205^{\circ}$ & $55^{\circ}$ & $90^{\circ}$ & 81 \\
\hline 1982 & 0425 & $33^{\circ} 55.80^{\prime}$ & $117^{\circ} 48.84^{\prime}$ & 12.3 & 3.1 & $20^{\circ}$ & $35^{\circ}$ & $90^{\circ}$ & 92 \\
\hline Sept 7,1982 & 2258 & $34^{\circ} 15.11^{\prime}$ & $117^{\circ} 56.86^{\prime}$ & 12.2 & 2.5 & $140^{\circ}$ & $10^{\circ}$ & $-180^{\circ}$ & 56 \\
\hline Sept. 11,1982 & 1355 & $33^{\circ} 48.33^{\prime}$ & $118^{\circ} 14.54^{\prime}$ & 4.6 & 2.6 & $200^{\circ}$ & $35^{\circ}$ & $100^{\circ}$ & 71 \\
\hline Sept. 17,1982 & 1057 & $33^{\circ} 55.59^{\circ}$ & $118^{\circ} 18.59^{\prime}$ & 15.1 & 3.3 & $45^{\circ}$ & $40^{\circ}$ & $90^{\circ}$ & 72 \\
\hline 1,1983 & 1448 & $33^{\circ} 53.01^{\circ}$ & $117^{\circ} 50.02^{\prime}$ & 5.7 & 2.6 & $333^{\circ}$ & $27^{\circ}$ & $46^{\circ}$ & 71 \\
\hline 7,1984 & 1018 & $33^{\circ} 28.65^{\prime}$ & $118^{\circ} 03.84^{\prime}$ & 9.3 & 4.0 & $230^{\circ}$ & $45^{\circ}$ & $90^{\circ}$ & 119 \\
\hline March 24,1984 & 1440 & $34^{\circ} 07.44^{\prime}$ & $118^{\circ} 32.73^{\prime}$ & 14.9 & 2.6 & $300^{\circ}$ & $57^{\circ}$ & $57^{\circ}$ & 75 \\
\hline May 8, 1984 & 2216 & $33^{\circ} 33.70^{\prime}$ & $118^{\circ} 14.73^{\circ}$ & 11.2 & 3.2 & $225^{\circ}$ & $15^{\circ}$ & $60^{\circ}$ & 99 \\
\hline 26,1984 & 1512 & $33^{\circ} 47.23^{\prime}$ & $118^{\circ} 30.48^{\prime}$ & 8.2 & 2.7 & $20^{\circ}$ & $41^{\circ}$ & $49^{\circ}$ & 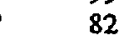 \\
\hline Sept. 12, 1984 & 0413 & $33^{\circ} 52.32^{\prime}$ & $117^{\circ} 56.57^{\prime}$ & 10.9 & 2.7 & $19^{\circ}$ & $43^{\circ}$ & $112^{\circ}$ & 90 \\
\hline Oct. 22, 1984 & 2212 & $33^{\circ} 41.91^{\prime}$ & $118^{\circ} 09.44^{\prime}$ & 8.0 & 2.7 & $135^{\circ}$ & $20^{\circ}$ & $130^{\circ}$ & 78 \\
\hline Dec. 14,1984 & 2128 & $33^{\circ} 52.91^{\prime}$ & $117^{\circ} 49.80^{\prime}$ & 6.8 & 3.1 & $357^{\circ}$ & $48^{\circ}$ & $108^{\circ}$ & 105 \\
\hline 1985 & 2322 & $33^{\circ} 41.94^{\prime}$ & $118^{\circ} 09.75^{\circ}$ & 9.8 & 3.3 & $235^{\circ}$ & $85^{\circ}$ & $130^{\circ}$ & 120 \\
\hline March 9,1985 & 0955 & $33^{\circ} 48.38^{\prime}$ & $118^{\circ} 31.51^{\circ}$ & 9.8 & 2.6 & $347^{\circ}$ & $39^{\circ}$ & $63^{\circ}$ & 90 \\
\hline Sept. 26, 1985 & 0125 & $33^{\circ} 56.65^{\prime}$ & $118^{\circ} 35.44^{\circ}$ & 10.3 & 2.5 & $42^{\circ}$ & $48^{\circ}$ & $108^{\circ}$ & 108 \\
\hline Dec. 19,1985 & 1303 & $34^{\circ} 02.18^{\prime}$ & $118^{\circ} 28.55^{\prime}$ & 14.0 & 2.8 & $56^{\circ}$ & $45^{\circ}$ & $80^{\circ}$ & 120 \\
\hline 9,1986 & 2241 & $34^{\circ} 06.84^{\prime}$ & $117^{\circ} 46.23^{\prime}$ & 5.6 & 3.5 & $343^{\circ}$ & $27^{\circ}$ & $46^{\circ}$ & 123 \\
\hline March 19, 1986 & 0247 & $33^{\circ} 44.14^{\prime}$ & $118^{\circ} 23.49^{\prime}$ & 4.7 & 2.7 & $324^{\circ}$ & $51^{\circ}$ & $47^{\circ}$ & 110 \\
\hline March 19.1986 & 1938 & $34^{\circ} 05.19^{\prime}$ & $118^{\circ} 25.14^{\prime}$ & 8,8 & 2.5 & $315^{\circ}$ & $10^{\circ}$ & $0^{\circ}$ & 78 \\
\hline 0,1986 & 0649 & $33^{\circ} 46.94^{\circ}$ & $118^{\circ} 18.42^{\prime}$ & 12.4 & 3. & $11^{\circ}$ & $45^{\circ}$ & $80^{\circ}$ & 137 \\
\hline 24,1986 & 0514 & $33^{\circ} 47.21^{\prime}$ & $118^{\circ} 18.11^{\prime}$ & 10.0 & 2.8 & $52^{\circ}$ & $36^{\circ}$ & $103^{\circ}$ & 102 \\
\hline April 5, 1986 & 0650 & $33^{\circ} 44.05^{\prime}$ & $118^{\circ} 00.74^{\prime}$ & 4.0 & 3.9 & $235^{\circ}$ & $50^{\circ}$ & $110^{\circ}$ & 149 \\
\hline 5,1986 & 1238 & $33^{\circ} 59.48^{\prime}$ & $118^{\circ} 43.28^{\prime}$ & 12.4 & 2.7 & $354^{\circ}$ & $51^{\circ}$ & $47^{\circ}$ & 121 \\
\hline 3,1986 & 1155 & $33^{\circ} 47.37^{\prime}$ & $118^{\circ} 17.84^{\prime}$ & 9.0 & 2.8 & $22^{\circ}$ & $39^{\circ}$ & $116^{\circ}$ & 74 \\
\hline 1986 & 0412 & $33^{\circ} 53.48^{\prime}$ & $118^{\circ} 23.44^{\circ}$ & 11.9 & 3.1 & $2^{\circ}$ & $36^{\circ}$ & $76^{\circ}$ & 121 \\
\hline May 20, 1986 & 0711 & $33^{\circ} 56.39^{\prime}$ & $118^{\circ} 39.54^{\circ}$ & 12.4 & 2.8 & $30^{\circ}$ & $45^{\circ}$ & $90^{\circ}$ & 110 \\
\hline 11,1986 & 1625 & $33^{\circ} 59.55^{\prime}$ & $118^{\circ} 41.95^{\circ}$ & 11.6 & 2.6 & $14^{\circ}$ & $43^{\circ}$ & $112^{\circ}$ & 72 \\
\hline 4,1986 & 0024 & $33^{\circ} 38.90^{\prime}$ & $117^{\circ} 32.16^{\prime}$ & 4.0 & 2.7 & $350^{\circ}$ & $40^{\circ}$ & $90^{\circ}$ & 108 \\
\hline 23,1986 & 1936 & $33^{\circ} 51.16^{\prime}$ & $118^{\circ} 29^{\circ} 42^{\prime}$ & 8.3 & 2.8 & $22^{\circ}$ & $39^{\circ}$ & $116^{\circ}$ & 80 \\
\hline July 30,1986 & 0114 & $34^{\circ} 00.02^{\prime}$ & $118^{\circ} 22.91^{\prime}$ & 4.5 & 2.8 & $39^{\circ}$ & $67^{\circ}$ & $135^{\circ}$ & 95 \\
\hline Aug. 24, 1986 & 1633 & $34^{\circ} 06.77^{\prime}$ & $117^{\circ} 45.76^{\circ}$ & 5.1 & 2.6 & $9^{\circ}$ & $52^{\circ}$ & $116^{\circ}$ & 98 \\
\hline 5.1986 & 060 & 33 & $2.92^{\prime}$ & 4.6 & 2.5 & $4^{\circ}$ & $57^{\circ}$ & $122^{\circ}$ & \\
\hline Oct. 6,1986 & 1156 & $33^{\circ} 35.95^{\prime}$ & $117^{\circ} 51.89^{\circ}$ & 13.4 & 2.5 & $180^{\circ}$ & $60^{\circ}$ & $60^{\circ}$ & 83 \\
\hline Oct. 14, 1986 & 0240 & $33^{\circ} 51.40^{\circ}$ & $118^{\circ} 33.66^{\circ}$ & 8.0 & 2.5 & $5^{\circ}$ & $20^{\circ}$ & $90^{\circ}$ & 80 \\
\hline May 5, 1987 & 1429 & $33^{\circ} 46.85^{\prime}$ & $117^{\circ} 33.48^{\prime}$ & 55 & 2.7 & $56^{\circ}$ & $27^{\circ}$ & $133^{\circ}$ & 83 \\
\hline June 8,1987 & 1229 & $33^{\circ} 46.46^{\prime}$ & $118^{\circ} 10.94^{\prime}$ & 12.8 & 3.2 & $205^{\circ}$ & $35^{\circ}$ & $90^{\circ}$ & 107 \\
\hline July 8,1987 & 1655 & $33^{\circ} 41.37^{\prime}$ & $118^{\circ} 13.91^{\circ}$ & 7.9 & 3.6 & $175^{\circ}$ & $50^{\circ}$ & $60^{\circ}$ & 118 \\
\hline July 8,1987 & 1857 & $33^{\circ} 41.12^{\prime}$ & $118^{\circ} 14.01^{\prime}$ & 4.7 & 2.8 & $190^{\circ}$ & $50^{\circ}$ & $70^{\circ}$ & 85 \\
\hline July 9,1987 & 0042 & $33^{\circ} 41.34^{\prime}$ & $118^{\circ} 14.13^{\prime}$ & 8.8 & 3.3 & $175^{\circ}$ & $50^{\circ}$ & $100^{\circ}$ & 120 \\
\hline Aug. 19, 1987 & 0940 & $33^{\circ} 35.59^{\prime}$ & $118^{\circ} 00.10^{\prime}$ & 5.8 & 3.0 & $245^{\circ}$ & $50^{\circ}$ & $100^{\circ}$ & 100 \\
\hline Oct. 1,1987 & 1442 & $34^{\circ} 02.96^{\prime}$ & $118^{\circ} 04.86^{\prime}$ & 14.6 & 5.9 & $0^{\circ}$ & $25^{\circ}$ & $90^{\circ}$ & 159 \\
\hline Oct. 5,1987 & 1437 & $33^{\circ} 53.86^{\prime}$ & $118^{\circ} 42.67^{\prime}$ & 14.8 & 2.7 & $57^{\circ}$ & $36^{\circ}$ & $103^{\circ}$ & 104 \\
\hline Oct. 17,1987 & 0925 & $33^{\circ} 59.32^{\prime}$ & $118^{\circ} 41.24^{\prime}$ & 9.0 & 2.7 & $355^{\circ}$ & $43^{\circ}$ & $67^{\circ}$ & 104 \\
\hline March 26, 1988 & 1454 & $33^{\circ} 59.38^{\circ}$ & $118^{\circ} 42.05^{\prime}$ & 13.1 & 3.7 & $27^{\circ}$ & $55^{\circ}$ & $83^{\circ}$ & 127 \\
\hline April 12,1988 & 1210 & $33^{\circ} 59.93^{\prime}$ & $118^{\circ} 10.61^{\prime}$ & 14.4 & 2.7 & $5^{\circ}$ & $40^{\circ}$ & $90^{\circ}$ & 100 \\
\hline April 13, 1988 & 0209 & $33^{\circ} 43.17^{\prime}$ & $118^{\circ} 44.56^{\prime}$ & 9.4 & 2.8 & $335^{\circ}$ & $40^{\circ}$ & $90^{\circ}$ & 94 \\
\hline April 26, 1988 & 1726 & $33^{\circ} 33.94^{\prime}$ & $118^{\circ} 16.99^{\prime}$ & 10.0 & 2.5 & $210^{\circ}$ & $20^{\circ}$ & $0^{\circ}$ & 61 \\
\hline
\end{tabular}


TABLE 1. (continued)

\begin{tabular}{|c|c|c|c|c|c|c|c|c|c|}
\hline $\begin{array}{l}\text { Origin } \\
\text { Day }\end{array}$ & $\begin{array}{l}\text { Time, } \\
\text { UT }\end{array}$ & $\begin{array}{l}\text { Latimde } \\
\mathrm{N}\end{array}$ & $\begin{array}{l}\text { Longitude } \\
\mathrm{W}\end{array}$ & $\begin{array}{l}\text { Depth, } \\
\text { km }\end{array}$ & $\begin{array}{l}\text { Mag. } \\
M_{L}\end{array}$ & \multicolumn{3}{|c|}{ Focal Mechanisms } & $\begin{array}{l}\text { Number of } \\
\text { First Motions }\end{array}$ \\
\hline \multicolumn{10}{|c|}{ Thrust Faulting Earthquakes (continued) } \\
\hline $\begin{array}{r}\text { June } 9,1988 \\
\text { Sept } 2,1988 \\
\text { Sept. } 9,1988 \\
\text { Sept. 12, } 1988 \\
\text { Nov. 20, } 1988 \\
\text { Jan. } 19,1989 \\
\text { June } 12,1989 \\
\text { June } 12,1989\end{array}$ & $\begin{array}{l}1723 \\
1748 \\
0452 \\
1324 \\
0539 \\
0653 \\
1657 \\
1722\end{array}$ & $\begin{array}{l}34^{\circ} 01.17^{\prime} \\
33^{\circ} 30.19^{\prime} \\
33^{\circ} 58.46^{\prime} \\
33^{\circ} 51.42^{\prime} \\
33^{\circ} 30.44^{\prime} \\
33^{\circ} 55.04^{\prime} \\
33^{\circ} 59.77^{\prime} \\
33^{\circ} 59.82^{\prime}\end{array}$ & $\begin{array}{l}118^{\circ} 13.66^{\prime} \\
118^{\circ} 04.46^{\prime} \\
118^{\circ} 45.73^{\prime} \\
118^{\circ} 26.12^{\prime} \\
118^{\circ} 04.46^{\prime} \\
118^{\circ} 37.37^{\prime} \\
118^{\circ} 10.71^{\prime} \\
118^{\circ} 10.33^{\prime}\end{array}$ & $\begin{array}{r}4.5 \\
11.0 \\
12.5 \\
12.3 \\
11.7 \\
13.8 \\
14.9 \\
15.1\end{array}$ & $\begin{array}{l}2.6 \\
2.5 \\
2.5 \\
3.9 \\
4.5 \\
5.0 \\
4.4 \\
4.1\end{array}$ & $\begin{array}{r}15^{\circ} \\
225^{\circ} \\
358^{\circ} \\
22^{\circ} \\
220^{\circ} \\
19^{\circ} \\
340^{\circ} \\
346^{\circ}\end{array}$ & $\begin{array}{l}51^{\circ} \\
70^{\circ} \\
20^{\circ} \\
56^{\circ} \\
45^{\circ} \\
45^{\circ} \\
41^{\circ} \\
31^{\circ}\end{array}$ & $\begin{array}{r}132^{\circ} \\
100^{\circ} \\
14^{\circ} \\
112^{\circ} \\
90^{\circ} \\
99^{\circ} \\
78^{\circ} \\
73^{\circ}\end{array}$ & $\begin{array}{r}109 \\
32 \\
55 \\
118 \\
121 \\
139 \\
70 \\
108\end{array}$ \\
\hline \multicolumn{10}{|c|}{ Normal Faulting Earthquakes } \\
\hline $\begin{array}{r}\text { Jan. } 24,1977 \\
\text { June } 29,1977 \\
\text { July } 9,1977 \\
\text { Aug. } 3,1977 \\
\text { Sept. } 26,1977 \\
\text { Oct. } 10,1977 \\
\text { March } 13,1978 \\
\text { Oct. } 30,1978 \\
\text { Dec. } 9,1978 \\
\text { Dec. } 31,1979 \\
\text { April } 14,1980 \\
\text { July } 29,1981 \\
\text { Aug. } 12,1981 \\
\text { Oct. } 27,1982 \\
\text { Nov. } 27,1982 \\
\text { Oct. } 16,1984 \\
\text { Feb. } 8,1987 \\
\text { Feb. 20, } 1987 \\
\text { May } 29,1987 \\
\text { June } 5,1987 \\
\text { July 7, } 1987 \\
\text { Dec. } 17,1988\end{array}$ & $\begin{array}{l}1135 \\
1358 \\
2022 \\
2208 \\
0835 \\
1238 \\
1638 \\
0740 \\
2301 \\
0603 \\
1153 \\
2339 \\
2258 \\
1021 \\
1752 \\
1749 \\
2008 \\
0033 \\
1516 \\
0325 \\
2107 \\
2346\end{array}$ & $\begin{array}{l}33^{\circ} 53.03^{\prime} \\
33^{\circ} 35.08^{\prime} \\
33^{\circ} 28.07^{\prime} \\
33^{\circ} 49.86^{\prime} \\
33^{\circ} 32.52^{\prime} \\
33^{\circ} 36.45^{\prime} \\
33^{\circ} 55.40^{\prime} \\
33^{\circ} 38.09^{\prime} \\
33^{\circ} 29.14^{\prime} \\
33^{\circ} 39.32^{\prime} \\
33^{\circ} 50.19^{\prime} \\
33^{\circ} 48.00^{\prime} \\
34^{\circ} 07.54^{\prime} \\
33^{\circ} 52.75^{\prime} \\
33^{\circ} 33.21^{\prime} \\
33^{\circ} 41.79^{\prime} \\
33^{\circ} 55.05^{\prime} \\
33^{\circ} 45.84^{\prime} \\
33^{\circ} 41.53^{\prime} \\
33^{\circ} 27.97^{\prime} \\
33^{\circ} 49.77^{\prime} \\
33^{\circ} 41.45^{\prime}\end{array}$ & $\begin{array}{l}118^{\circ} 11.64^{\prime} \\
117^{\circ} 38.04^{\prime} \\
118^{\circ} 01.86^{\prime} \\
118^{\circ} 08.56^{\prime} \\
118^{\circ} 13.93^{\prime} \\
118^{\circ} 04.30^{\prime} \\
117^{\circ} 59.23^{\prime} \\
118^{\circ} 24.14^{\prime} \\
118^{\circ} 16.88^{\prime} \\
117^{\circ} 54.90^{\prime} \\
118^{\circ} 25.83^{\prime} \\
118^{\circ} 42.75^{\prime} \\
118^{\circ} 36.49^{\prime} \\
118^{\circ} 11.92^{\prime} \\
118^{\circ} 12.39^{\prime} \\
118^{\circ} 10.04^{\prime} \\
118^{\circ} 16.80^{\prime} \\
118^{\circ} 42.82^{\prime} \\
118^{\circ} 09.48^{\prime} \\
118^{\circ} 15.61^{\prime} \\
118^{\circ} 09.91^{\prime} \\
118^{\circ} 07.00^{\prime}\end{array}$ & $\begin{array}{r}10.4 \\
5.5 \\
6.5 \\
7.1 \\
9.4 \\
8.8 \\
3.8 \\
17.7 \\
11.8 \\
8.7 \\
9.1 \\
9.6 \\
3.3 \\
8.8 \\
5.8 \\
7.7 \\
13.6 \\
11.5 \\
4.5 \\
7.5 \\
6.8 \\
4.2\end{array}$ & $\begin{array}{l}2.7 \\
2.8 \\
2.5 \\
2.5 \\
2.7 \\
3.0 \\
3.3 \\
2.5 \\
2.6 \\
2.7 \\
2.5 \\
3.9 \\
2.7 \\
2.9 \\
3.1 \\
3.1 \\
2.5 \\
2.5 \\
3.1 \\
2.6 \\
3.2 \\
3.2\end{array}$ & $\begin{array}{r}110^{\circ} \\
235^{\circ} \\
110^{\circ} \\
135^{\circ} \\
180^{\circ} \\
31^{\circ} \\
287^{\circ} \\
285^{\circ} \\
150^{\circ} \\
90^{\circ} \\
277^{\circ} \\
235^{\circ} \\
129^{\circ} \\
80^{\circ} \\
230^{\circ} \\
260^{\circ} \\
95^{\circ} \\
235^{\circ} \\
33^{\circ} \\
85^{\circ} \\
90^{\circ} \\
8^{\circ}\end{array}$ & $\begin{array}{r}50^{\circ} \\
41^{\circ} \\
40^{\circ} \\
40^{\circ} \\
70^{\circ} \\
45^{\circ} \\
39^{\circ} \\
5^{\circ} \\
50^{\circ} \\
50^{\circ} \\
48^{\circ} \\
5^{\circ} \\
67^{\circ} \\
55^{\circ} \\
0^{\circ} \\
5^{\circ} \\
40^{\circ} \\
45^{\circ} \\
66^{\circ} \\
45^{\circ} \\
50^{\circ} \\
78^{\circ}\end{array}$ & $\begin{array}{r}-80^{\circ} \\
-130^{\circ} \\
-110^{\circ} \\
-70^{\circ} \\
-60^{\circ} \\
-99^{\circ} \\
-63^{\circ} \\
-60^{\circ} \\
-110^{\circ} \\
-130^{\circ} \\
-71^{\circ} \\
-70^{\circ} \\
-69^{\circ} \\
-120^{\circ} \\
-89^{\circ} \\
-50^{\circ} \\
-130^{\circ} \\
-130^{\circ} \\
-99^{\circ} \\
-130^{\circ} \\
-110^{\circ} \\
-56^{\circ}\end{array}$ & $\begin{array}{r}60 \\
48 \\
46 \\
54 \\
53 \\
78 \\
58 \\
56 \\
58 \\
63 \\
44 \\
108 \\
67 \\
75 \\
87 \\
96 \\
75 \\
41 \\
103 \\
78 \\
50 \\
130\end{array}$ \\
\hline
\end{tabular}

TABLE 2. Significant Earthquakes in the Greater Los Angeles Basin and Adjacent Offshore Regions

\begin{tabular}{llll}
\hline Date & Earthquake & $M_{L}$ & Reference \\
\hline June 21, 1920 & Inglewood & 4.9 & Taber [1920] \\
Aug. 30, 1930 & Santa Monica Bay & 5.2 & Gutenberg et al. [1932] \\
March 10, 1933 & Long Beach & 6.3 & Wood [1933] \\
May 31, 1938 & Elsinore Fault & 5.5 & Hileman et al. [1973] \\
Oct. 22, 1941 & Gardena & 4.9 & Richter [1958] \\
Nov. 14, 1941 & Torrance-Gardena & 5.4 & Richter [1958] \\
Sept. 12, 1970 & Lytle Creck & 5.4 & Jones [1984] \\
Feb. 9, 1971 & San Fernando & 6.4 & Whitcomb et al. [1973] \\
Feb. 21, 1973 & Point Mugu & 5.9 & Stierman and Ellsworth [1972] \\
Jan. 1, 1979 & Malibu & 5.0 & Hauksson and Saldivar [1986] \\
Sept. 4, 1981 & Santa Barbara Island & 5.3 & Corbett [1984] \\
Oct. 1, 1987 & Whittier Narrows & 5.9 & Hauksson et al. [1988] \\
Dec. 3, 1988 & Pasadena & 4.9 & Jones et al. [1990] \\
Jan. 19, 1989 & Malibu & 5.0 & E. Hauksson [manuscript in preparation, 1990] \\
\hline
\end{tabular}

$\left(M_{L}=4.9\right)$ Pasadena earthquake showed left-lateral movement on the Raymond fault [Jones et al., 1990]. Farther to the northwest, three right-lateral strike-slip events have occurred along the northwest striking surface trace of the Verdugo fault. Similar to the Pasadena earthquake, the $1988\left(M_{L}=4.6\right)$ Upland sequence showed mostly left-lateral strike-slip faulting, on the northeast striking San Jose fault (Figure 10). The San Jose fault thus may provide for a left step in the frontal fault system.
The mappable surficial late Quaternary faults and the 144 strike-slip faulting events of $M \geq 2.5$ show that strike-slip faulting is a significant mode of deformation in the Los Angeles basin. The strike-slip faults appear to accommodate lateral movements of crustal blocks.

\section{Thrust Faulting}

As can be seen in Figure 11, the thrust focal mechanisms are not located adjacent to the north dipping 

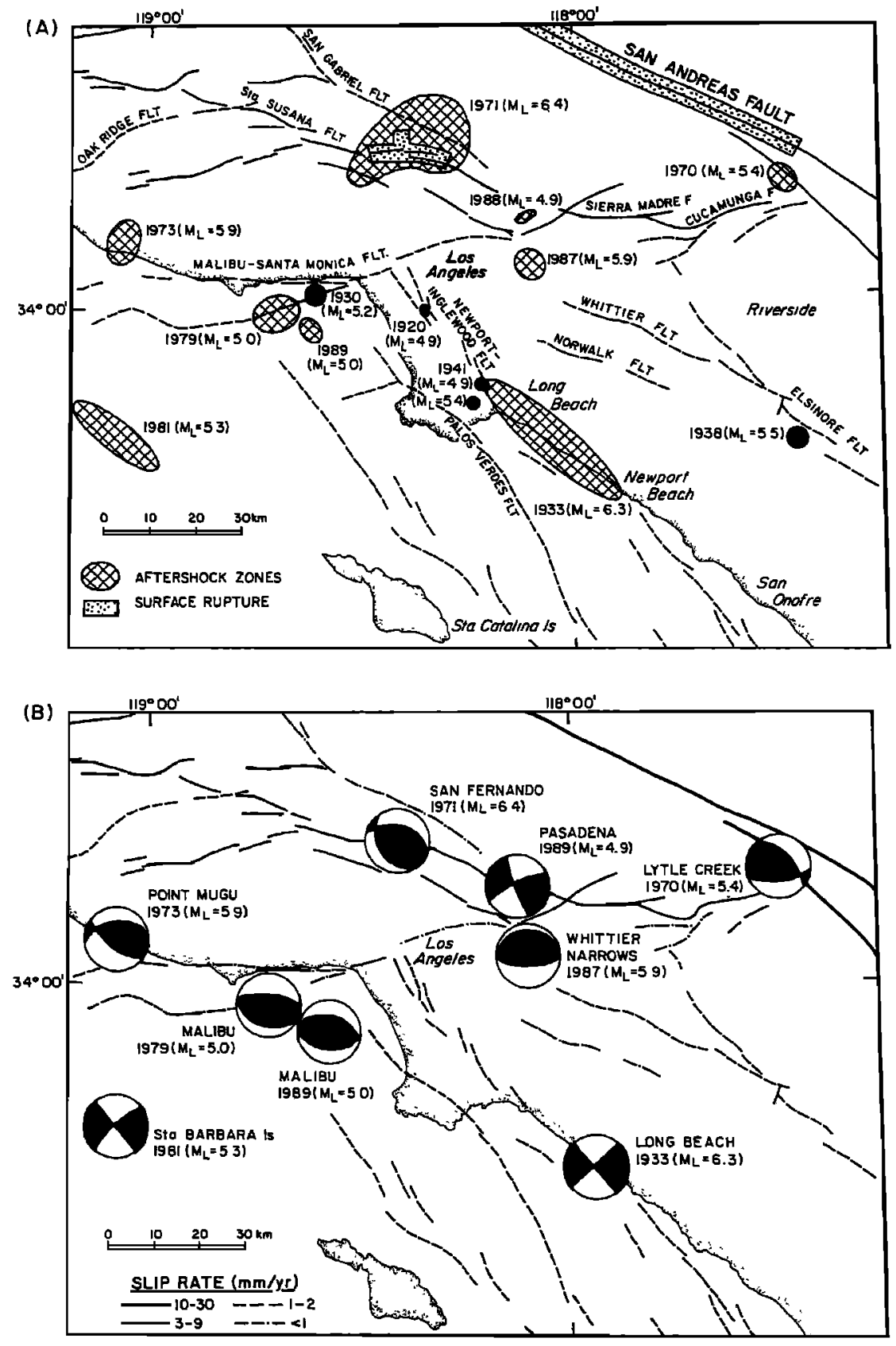

Fig. 6. (a) Significant earthquakes of $M \geq 4.9$ that have occurred in the greater Los Angeles basin area since 1920. Aftershock zones are shaded with cross hatching. Dotted areas indicate surface rupture, including the rupture of the 1857 earthquake along the San Andreas fault. (b) Single-event lower hemisphere focal mechanisms of the $M \geq 4.9$ events in the greater Los Angeles basin. Focal mechanisms are from WoodwardClyde Consultants [1979], Jones [1984], Whitcomb et al. [1973], Stierman and Ellsworth [1976], Corbett [1984], Hauksson and Saldivar [1986], Hauksson and Jones [1989], and Jones et al. [1990].

surficial reverse faults, such as the Santa Monica and Sierra Madre faults that define the north edge of the sedimentary basin. Instead the thrust or reverse focal mechanisms are, in general, south of these reverse faults, forming broad clusters along the flanks of the basin. This coincides with most of the folding of the basin sediments (Figure $9 b$ ). This spatial coincidence of the folding and thrust faulting can be explained with fault-bend or fault-propagation folding models [e.g., Suppe, 1985], where the thrust faulting earthquakes at depth cause folding of the cover sequence. This was demonstrated by the coseismic uplift of sediment in the 1987 Whittier Narrows earthquake [Lin and Stein, 1989; Davis et al., 1989]. Thus, combining the geological evidence for folding and the seismological evidence for thrust faulting, two fold and thrust belts can be identified along the flanks of the basin (Figure 11). These belts are called here the Elysian Park and the TorranceWilmington fold and thrust belts and are located along the east and north and southwest flanks of the basin, respectively. 
(a)

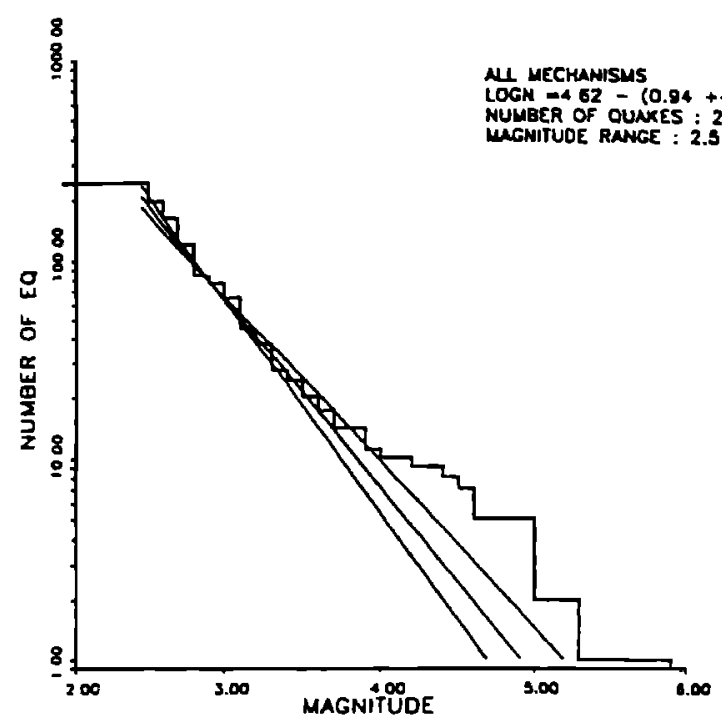

(b)

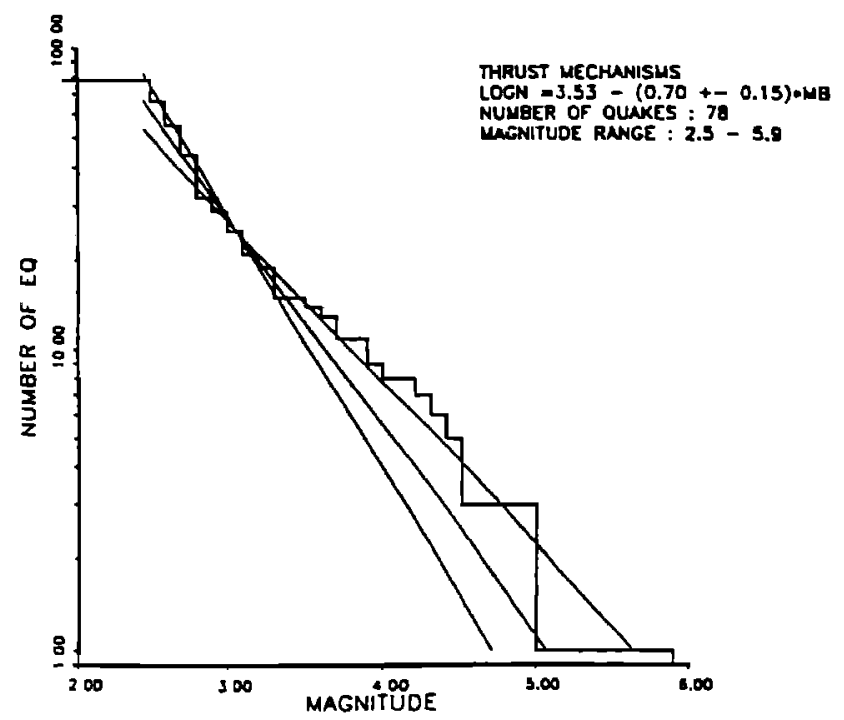

(c)

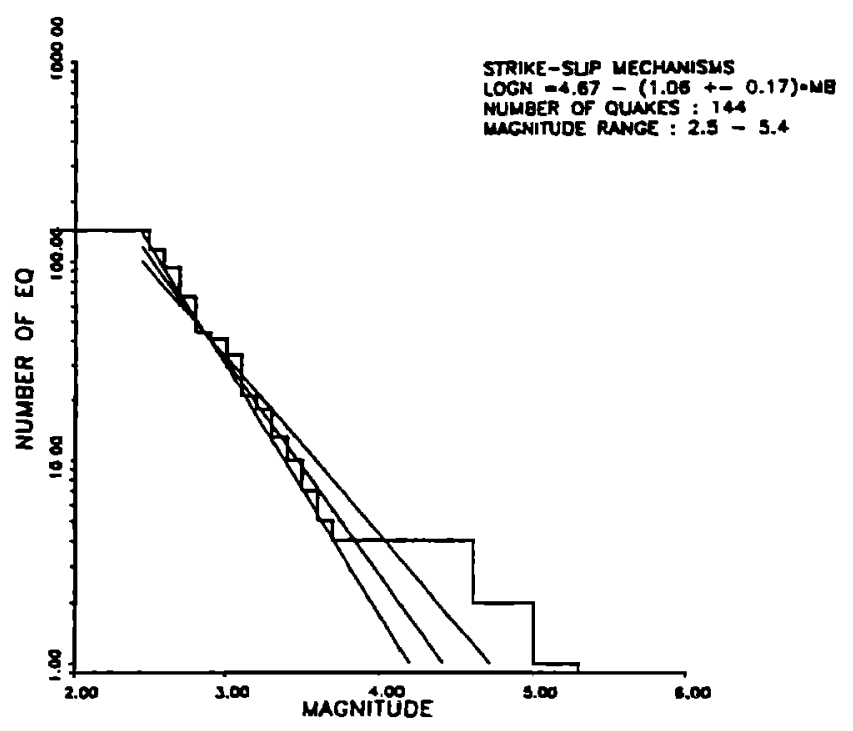

(d)

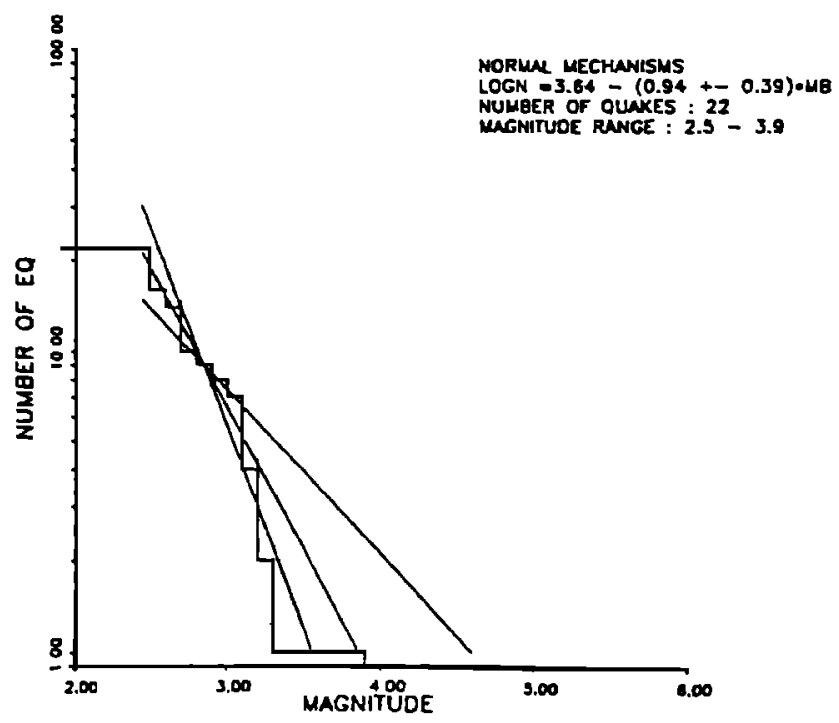

Fig. 7. (a) The $b$ value from the Gutenberg-Richter relationship for the 244 events of $M \geq 2.5$ from 1977 to 1989. The number of events included in the $b$ value determination and magnitude range are also shown. The three lines are the best fit, the best fit minus one standard deviation in the $b$ value, and the best fit plus one standard deviation in the $b$ value. ( $b$ ) The $b$ value for only the thrust faulting events. (c) The $b$ value for only the strike-slip faulting events. (d) The $b$ value for only the normal faulting events.

Within the eastern part of the Elysian Park fold and thrust belt the thrust faulting focal mechanisms form a zone dipping $25^{\circ}-35^{\circ}$ north (Figure 12 ). It is not possible to tell, based on hypocentral depth distribution and focal mechanisms alone, if this zone consists of one continuous fault or many small abutting or overlapping faults. This zone of thrust faulting is closest to the surface at $4.6 \mathrm{~km}$ depth, near Yorba Linda. The southemmost mapped thrust fault along the eastern flank of the Los Angeles basin, the Peralta Hills thrust fault is located in this area, $5 \mathrm{~km}$ south of Yorba Linda [Bryant and Fife, 1982]. The presence of both the shallow thrust events and the surface exposure of the Peralta Hills thrust fault near Yorba Linda suggest that in rare cases the concealed thrusts can cause surface ruptures. Recently Wright [1990] has suggested independently that the Peralta Hills thrust may be associated with the Elysian Park thrust zone.

The largest earthquake in this data set is the 1987 $\left(M_{L}=5.9\right)$ Whittier Narrows earthquake that ruptured a 5km-long segment of the Elysian Park fold and thrust belt. The Elysian Park fold and thrust belt extends to the west of the Whittier Narrows, beneath downtown Los Angeles, and 


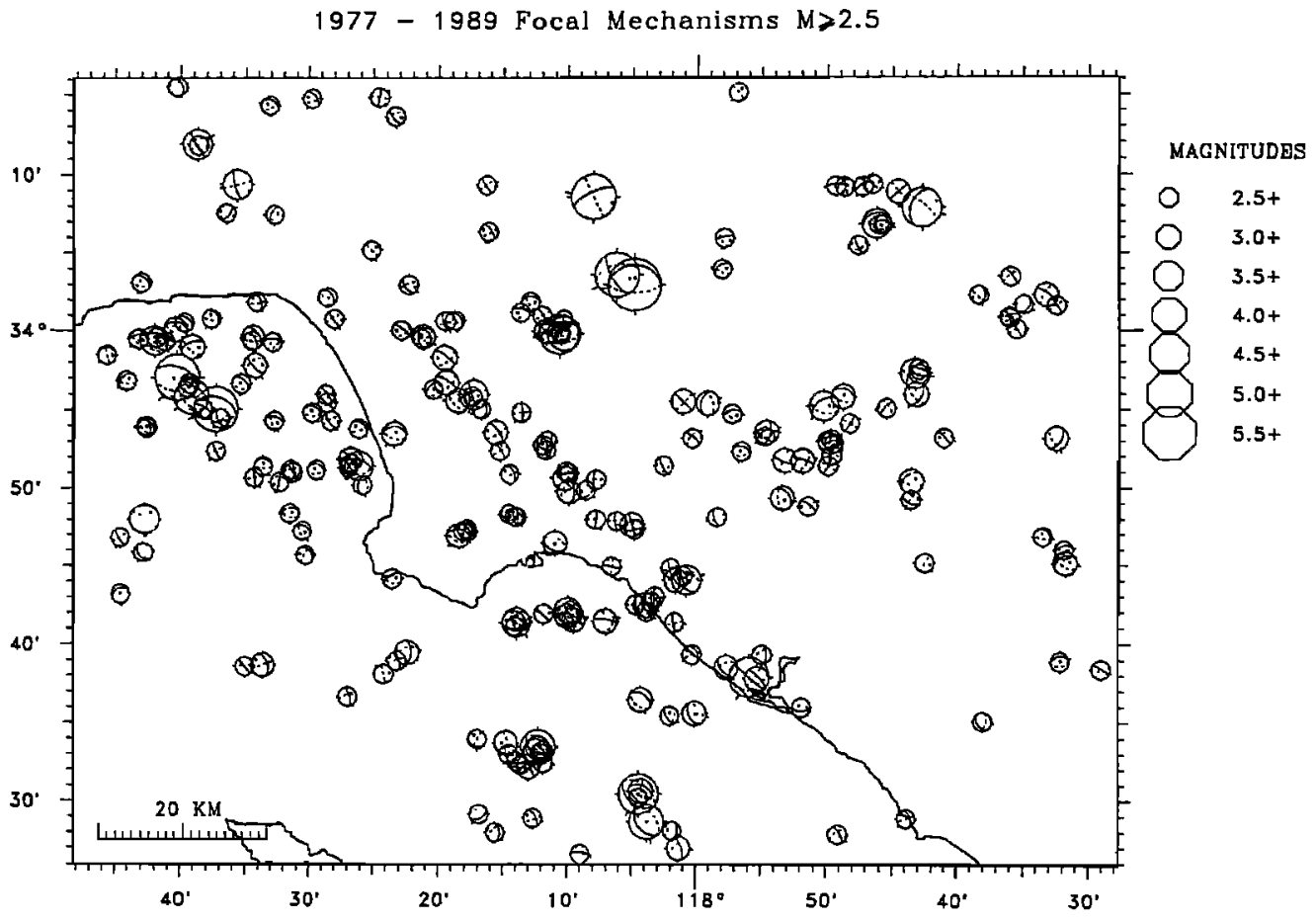

Fig. 8. The 244 single-event lower hemisphere focal mechanisms determined for earthquakes from 1977 to 1989 of $M \geq 2.5$. The solid nodal plane in each mechanism is the one chosen for stress inversions, while the dashed nodal plane is the auxiliary plane.

continues into Santa Monica Bay, where it is found at a depth of 10-15 km (Figure 11). It may be offset or segmented by the northem end of the Newport-Inglewood fault, because the maximum focal depths to the east of the intersection of the two faults are 3-4 km deeper than to the west (Figure 12).

The Elysian Park fold and thrust belt is cross cut by several strike-slip faults. The most prominent of these strike-slip faults is the Whittier fault that has had up to 30 km of right-lateral slip since 9-4 Ma [Lamar, 1990]. The vertical offset on it is more difficult to quantify but is much less and averages about 1.0-1.5 km [Wright, 1990]. The Elysian Park thrust zone has moved 5.5-21 km since 4.0-2.2 Ma [Davis et al., 1989]. Because it is difficult to analyze the coexistence of these two systems of faulting in the same area, previous investigators have simply stated that the strike-slip faulting was primary [e.g., Wilcox et al., 1973; Lamar, 1990], or that the thrusting was primary [e.g., Davis et al., 1989]. The evidence presented in this paper suggests that both strike-slip and thrust faulting are active simultaneously.

The Torrance-Wilmington fold and thrust belt is oullined by fold axes and thrust focal mechanisms extending from offshore Newport Beach, across the Palos Verdes Peninsula into Santa Monica Bay along the southwest flank of the basin (Figure 11). The three cross sections in Figure 13 show that the depth distribution of thrust faulting events within this fold and thrust belt is more complex than within the Elysian Park fold and thrust belt. The cross section C-C' shows a zone of thrust faulting dipping north $30^{\circ}-40^{\circ}$, while the cross sections D-D' and E-E' show a steeply dipping zone beneath the Palos Verdes fault trace and a zone in the San Pedro Bay dipping southwest $20^{\circ}$. $30^{\circ}$.
In Santa Monica Bay the two thrust belts merge into a north dipping imbricate thrust zone. The Elysian Park thrust zone is on the north side, beneath the Malibu shelf. On the south side, the north dipping thrust zone of the Torrance-Wilmington belt changes strike from northwest to west as it merges with the Elysian Park zone. The Shelf Projection anticline in Santa Monica Bay is probably a surface expression of this imbricate thrust zone [Hauksson and Saldivar, 1989].

The Palos Verdes fault coincides with the TorranceWilmington fold and thrust belt along most of its length (Figure 9) and has been either interpreted to be a rightlateral strike-slip fault or an oblique steeply dipping reverse fault [e.g., Ziony and Yerkes, 1985; Davis et al., 1989]. Although numerous efforts have been made to find horizontal surficial offsets on the Palos Verdes fault, no clear evidence has been found [Darrow and Fischer, 1983]. Vertical offsets have been documented from uplifted shore terraces and show a vertical slip rate of $0.1-0.4 \mathrm{~mm} / \mathrm{yr}$ [Darrow and Fischer, 1983]. Davis et al. [1989] assumed negligible lateral motion since $2-4 \mathrm{Ma}$ on the Palos Verdes fault and modeled a concealed thrust fault beneath Palos Verdes with a slip rate of $1.9-3.5 \mathrm{~mm} / \mathrm{yr}$. In this study, strike-slip events have only been found near the Palos Verdes fault as it heads offshore, both in San Pedro Bay and in Santa Monica Bay. Because almost no evidence for strike-slip motion has been found (Figure 10) and the vertical motion appears to be small, the Palos Verdes fault thus appears to accommodate little of the total convergence in the southwest flank of the basin. Alternatively, moderate-sized or large earthquakes on the Palos Verdes fault may rarely rupture up to the surface.

Because most faults in the study area are either north or northeast dipping, the south dipping thrust in San Pedro 

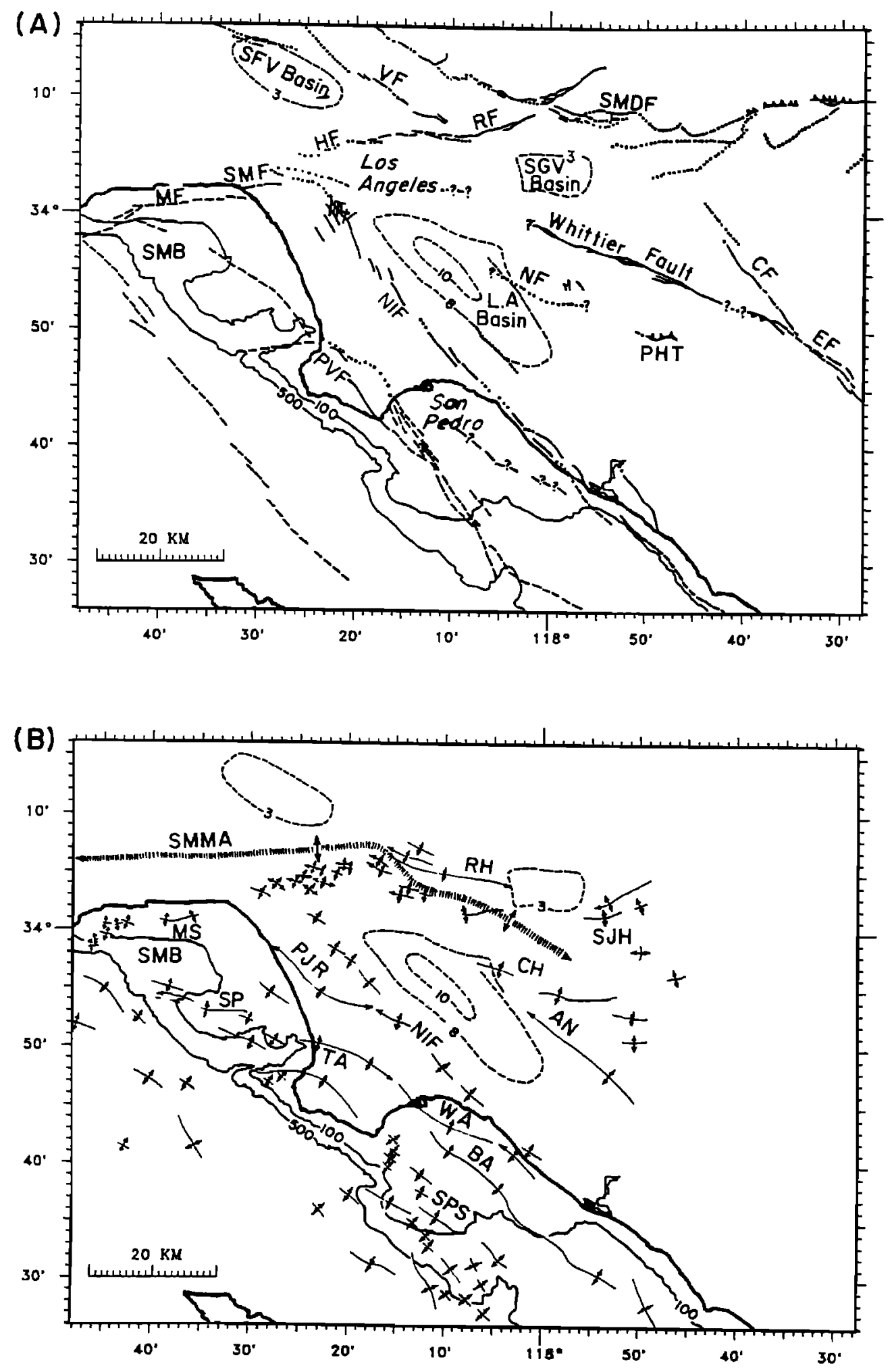

Fig. 9. (a) Late Quaternary faults in the Los Angeles basin [Ziony and Jones, 1989]. Shown as solid lines where the surface trace has been mapped and as dashed lines where inferred. SFV, San Femando Valley; SMDF, Sierra Madre fault; SGV, San Gabriel Valley; LA Basin, Los Angeles basin; NIF, Newport-Inglewood fault; PVF, Palos Verdes fault; SMB, Santa Monica Bay; MF, Malibu fault; HF, Hollywood fault; RF, Raymond fault; CF, Chino fault; EF, Elsinore fault; NF, Norwalk fault; PHT, Peralta hills thrust fault; and VF, Verdugo fault. (b) Late Quaternary fold axes in the Los Angeles basin [Yerkes, 1965; Wright, 1987; Harding and Tuminas, 1988; Davis et al., 1989]. SMMA, Santa Monica Mountains Anticline; RH, Raymond Hill; SJH, San Jose Hills; CH, Chino Hills; AN, Anaheim Nose; PJR, Playa-del Ray; TA, Torrance anticline; WA, Wilmington anticline; BA, Beta anticline; SPS, San Pedro Shelf; MS, Malibu Shelf; SMB, Santa Monica Bay; SP, Shelf Projection in Santa Monica Bay. 


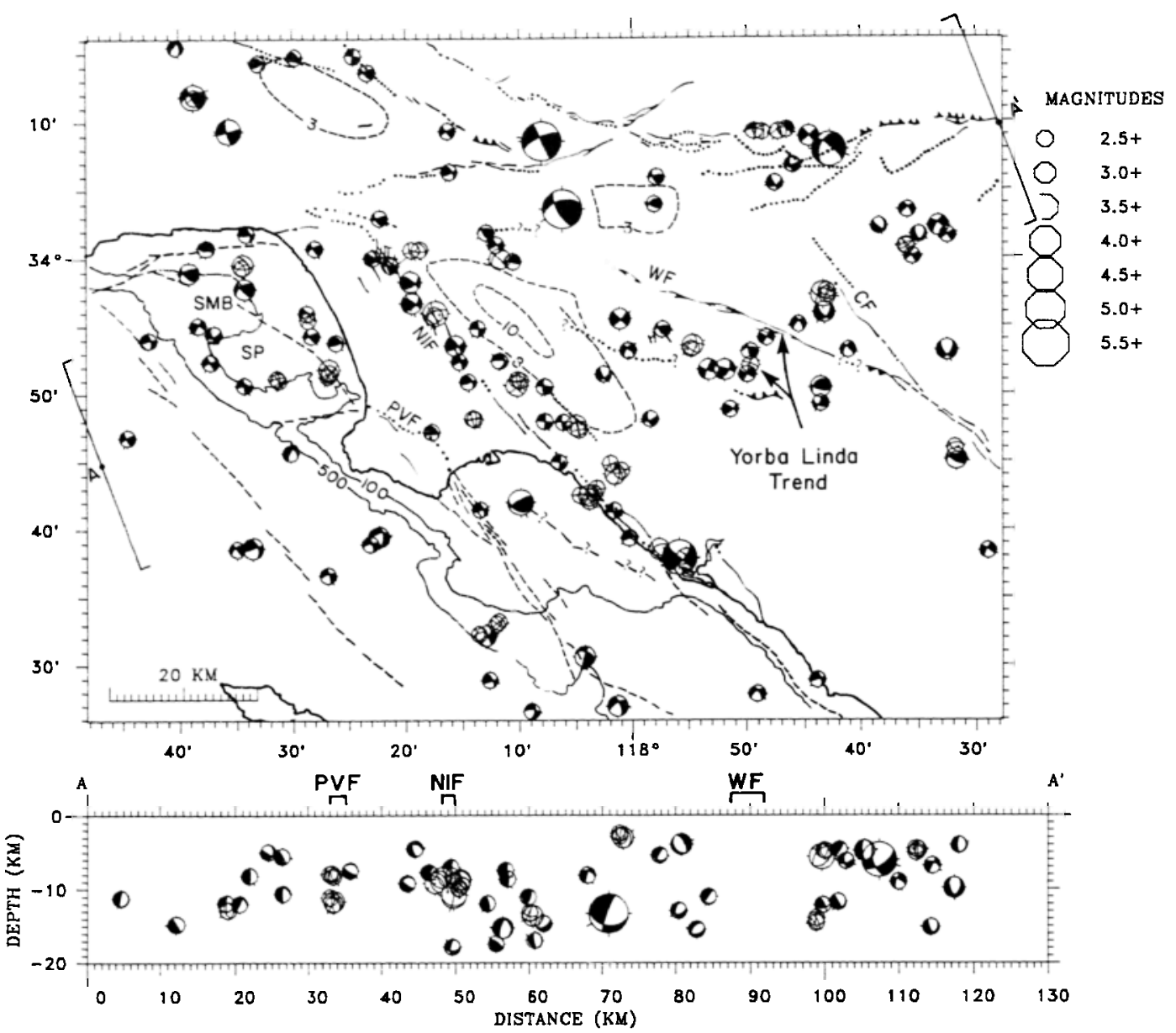

Fig. 10. (Top) Map of late Quaternary faults and strike-slip focal mechanisms. Compressional quadrants are shaded. Mechanisms that overlap significantly are not shaded. WF, Whittier fault; CH, Chino fault; NIF, Newport-Inglewood fault; PVF, Palos Verdes fault; SMB, Santa Monica Bay; SP, Shelf Projection. Yorba Linda trend is the northeast trend of focal mechanisms. (Bottom) A northeast trending depth section A-A:

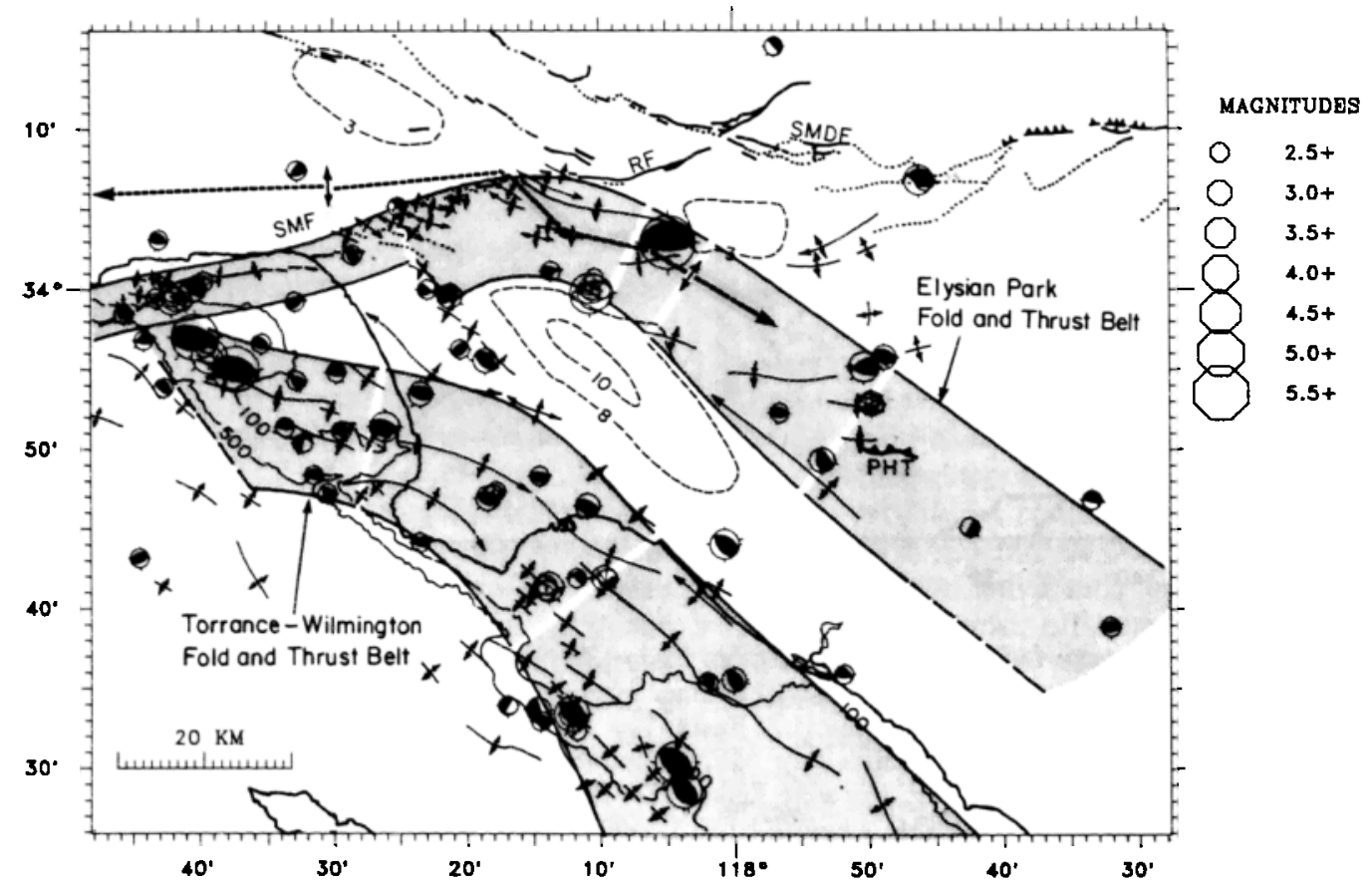

Fig. 11. Map of fold axes and thrust focal mechanisms. The Elysian Park and Torrance-Wilmington fold and thrust belts are shaded. Breaks in the shading are at segment boundaries. RF, Raymond fault; SMDF, Sierra Madre fault; SMF, Santa Monica fault; and PHT, Peralta Hills thrust. 

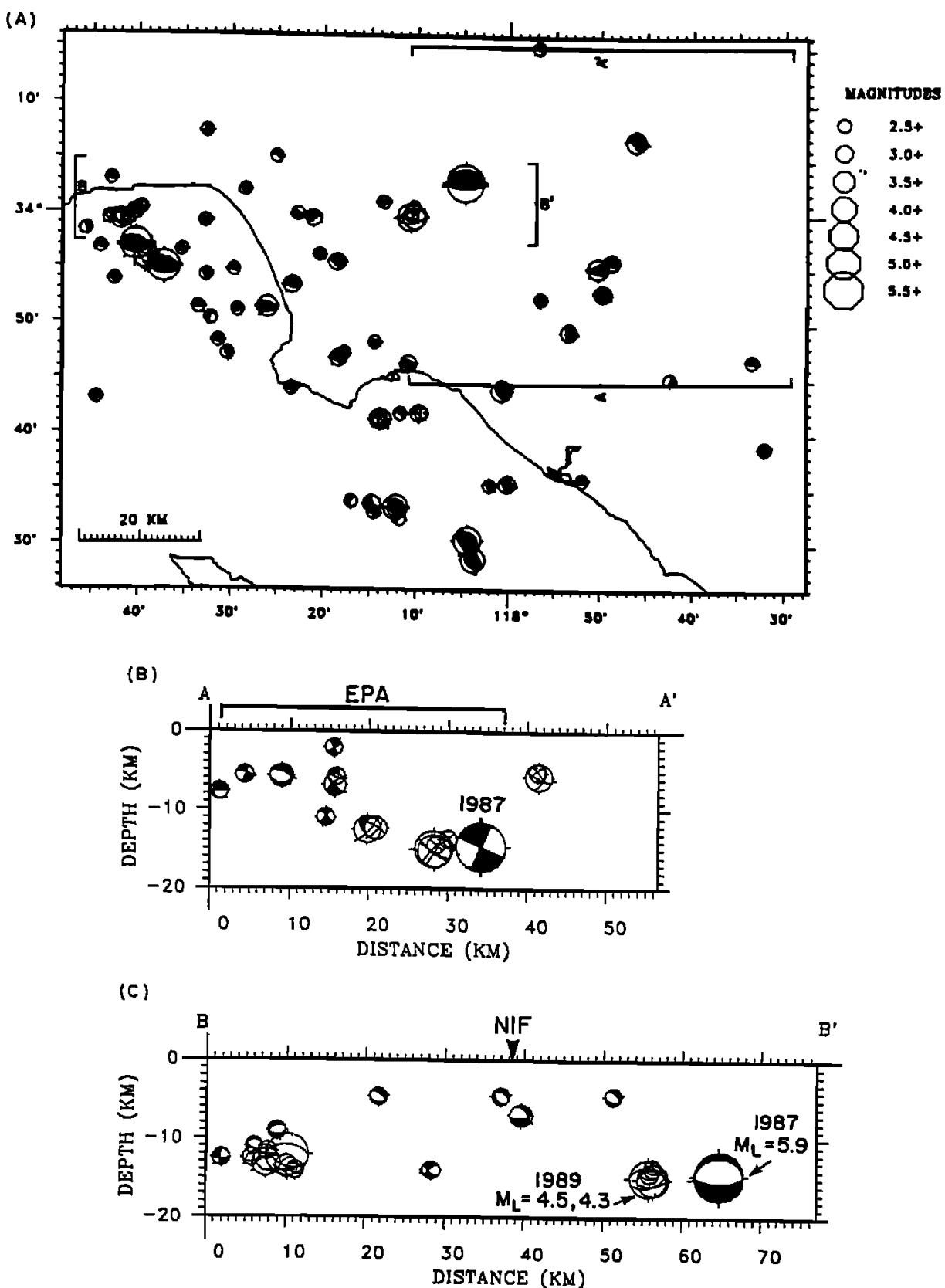

Fig. 12. (a) Map showing thrust focal mechanisms and location of depth sections. (b) North-south depth section A-A' along the Elysian Park fold and thrust belt. (c) East-west depth section B-B' showing the offset in
depth of thrust earthquakes near the Newport-Inglewood fault.

Bay is unexpected and requires a special explanation. One possible explanation for this zone of thrusting, which has two $M_{L}=4.0$ and 4.5 events near its downdip end, could be a back thrust, which often appears at the later stages of thrust faulting (J. Suppe, personal communication, 1989). Usually a set of thrust faults is configured such that on a regional scale the upper block is overriding the lower block, while often a conjugate set of thrust faults, called back thrusts, may develop. In San Pedro Bay, this implies a deeper east or northeast dipping thrust fault, which would be the primary member of the conjugate set.

To evaluate the earthquake potential of these thrust zones, some constraints on their widths are needed. Moderate-sized earthquakes in most cases nucleate near the bottom of a fault zone, although large or great earthquakes can rupture down into the semibrittle region [Scholz, 1988]. The 1987 Whittier Narrows earthquake was located near the downdip end of the Elysian Park thrust belt. Similarly, the 1979 and 1989 Malibu earthquakes, which are the largest events to occur in Santa Monica Bay since 1932, are located near the downdip end of the TorranceWilmington thrust belt. The most shallow thrust events occur in the depth range of 4-6 km. Thus the thrust zones extend from shallow depths of 4-6 km down to depths of $14-16 \mathrm{~km}$. If a $30^{\circ} \mathrm{dip}$ is assumed the width range is $20-24$ $\mathrm{km}, 30-50 \%$ wider than the seismogenic width of most strike-slip faults in southern Califomia.

Davis et al. [1989] proposed that most of the thrust faulting in the basin, including the Whittier Narrows earthquake, occurs on crustal ramps that form structural and 
(A)
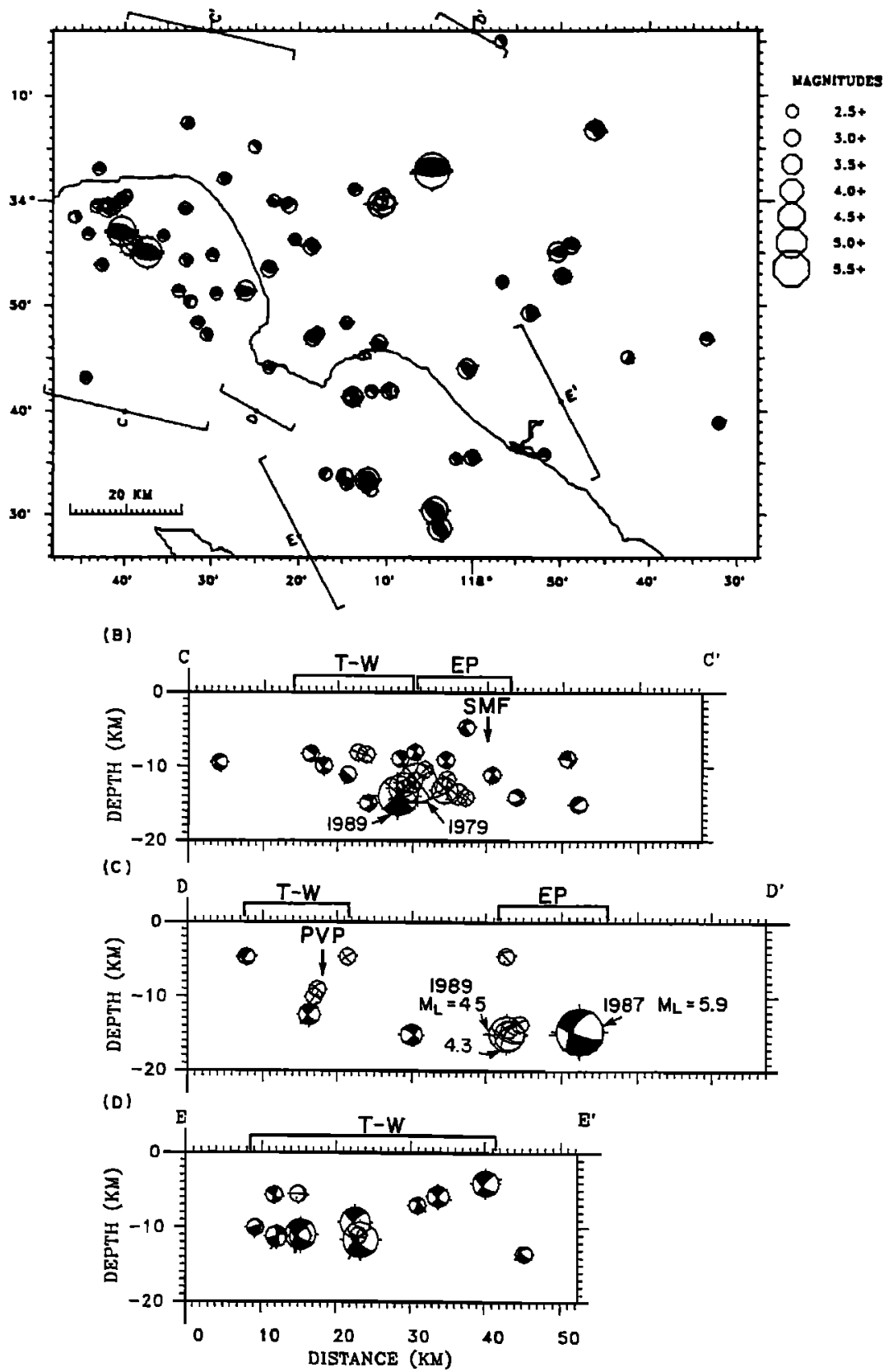

Fig. 13. (a) Map showing thrust focal mechanisms and locations of depth sections. (b) North-south depth section C-C' in Santa Monica Bay, includes earthquakes from both the Torrance-Wilmington and the Elysian Park thrust belts. T-W, Torrance-Wilmington fold and thrust belt; EP, Elysian Park fold and thrust belt. (c) Northeast trending depth section D-D' across the Palos Verdes Peninsula showing a cluster of steeply dipping events near the Palos Verdes fault. (d) East trending depth section E-E' in San Pedro Bay showing a west dipping thrust zone.

topographic highs on a regional subhorizontal, detachment surface. The complex distribution of thrust faulting earthquakes beneath the flanks of the basin reported here is not obviously compatible with such a model. For instance, the dipping zone of thrust faulting seen in the cross section in Figure $12 b$ extending from 16 to $4 \mathrm{~km}$ depth, is too steep to be the detachment itself and too long to be a simple crustal ramp. Although a subhorizontal regional detachment surface without crustal ramps may exist beneath the deep basin and the flanks, existing dipping fault zones within the flanks appear to accommodate most of the brittle-elastic compressional deformation.

\section{Normal Faulting}

Normal faulting is observed in $9 \%$ of the earthquakes analyzed, a small fraction of the ongoing faulting (Table 1). The normal faulting events all have small magnitudes with the largest event of $M_{L}=3.9$. The normal faulting mechanisms in Figure 14 are distributed adjacent to the Newport-Inglewood fault and offshore in the San Pedro Bay. The offshore events may be less well constrained 

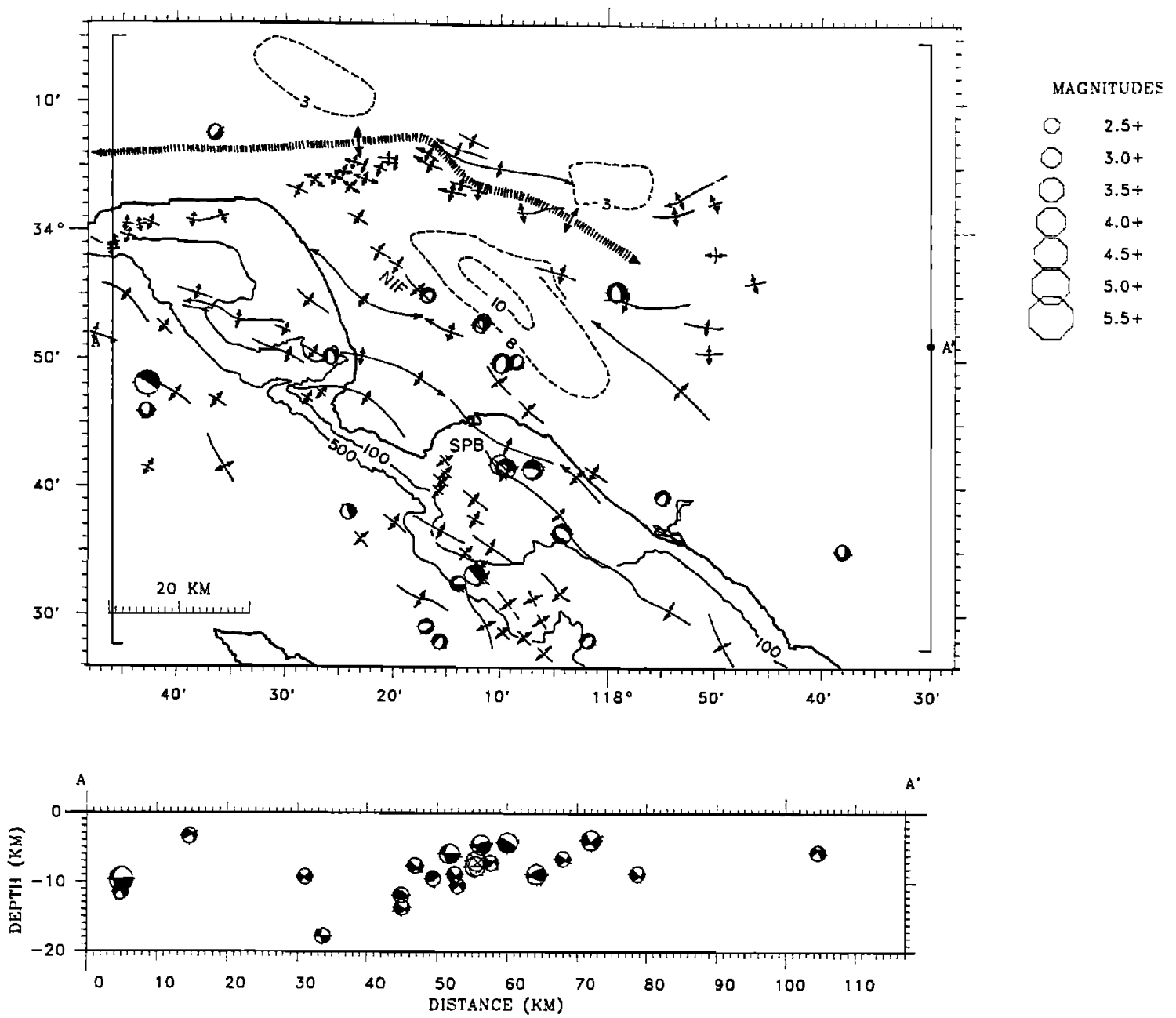

Fig. 14. (Top) Map of fold axes and normal faulting focal mechanisms. (Bottom) an east trending depth section A-A'. NIF, Newport-Inglewood fault, and SPB, San Pedro Bay.

than the onshore events because the distance to the nearest station is in some cases larger than one focal depth. The normal faulting may be associated with extensional bending moments that can cause extensional faulting during the growth of anticlines [e.g., Ramsey and Huber, 1987]. Altematively, the normal faulting events with nodal planes trending northwest to north-northwest could be related to faulting on planes orthogonal to the axes of anticlines. Because the anticlines usually plunge at the ends to maintain continuity with the unfolded beds, normal faulting could occur to accommodate this deformation as is often seen in seismic reflection profiles (J. Suppe personal communication, 1989).

If the lower crust beneath the Los Angeles basin was still subsiding, the normal focal mechanisms could be expected to be deep events associated with this downfaulting of the lower crust. This is not observed in the cross section in Figure 14. Instead, the foci of the normal faulting earthquakes are shallow and appear to be associated with geological processes in the upper crust such as growth of folds or sediment loading of the shallow crust.

In summary, active faulting in the Los Angeles basin includes strike-slip, thrust, and a few cases of normal faulting. The coexistence of strike-slip and thrust faulting suggests that crustal thickening and lateral movement of crustal blocks are both important mechanisms for accommodating crustal shortening within the basin. The normal faulting is secondary and appears to accommodate local geometrical adjustments within compressional geologic structures.

\section{STATE OF STREss}

The seismicity, active faulting, and folding in the Los Angeles basin are driven by a regional tectonic stress field. In Figure 15, the $P$ and $T$ axes from the 244 focal mechanisms are shown along with six $P$ axes determined from borehole breakouts by Mount [1989]. Close correspondence between the two data sets is shown in the consistent local variations in the orientations of the $P$ axes. For instance, at the northem end of the Newport-Inglewood fault both the thrust focal mechanisms and the borehole breakout data show northeast to east-northeast trending $P$ axes. This suggests that the trends of the principal stress axes do not change significantly with depth and that borehole breakouts in the Los Angeles basin although taken from shallow depth are representative of the tectonic stress field. 


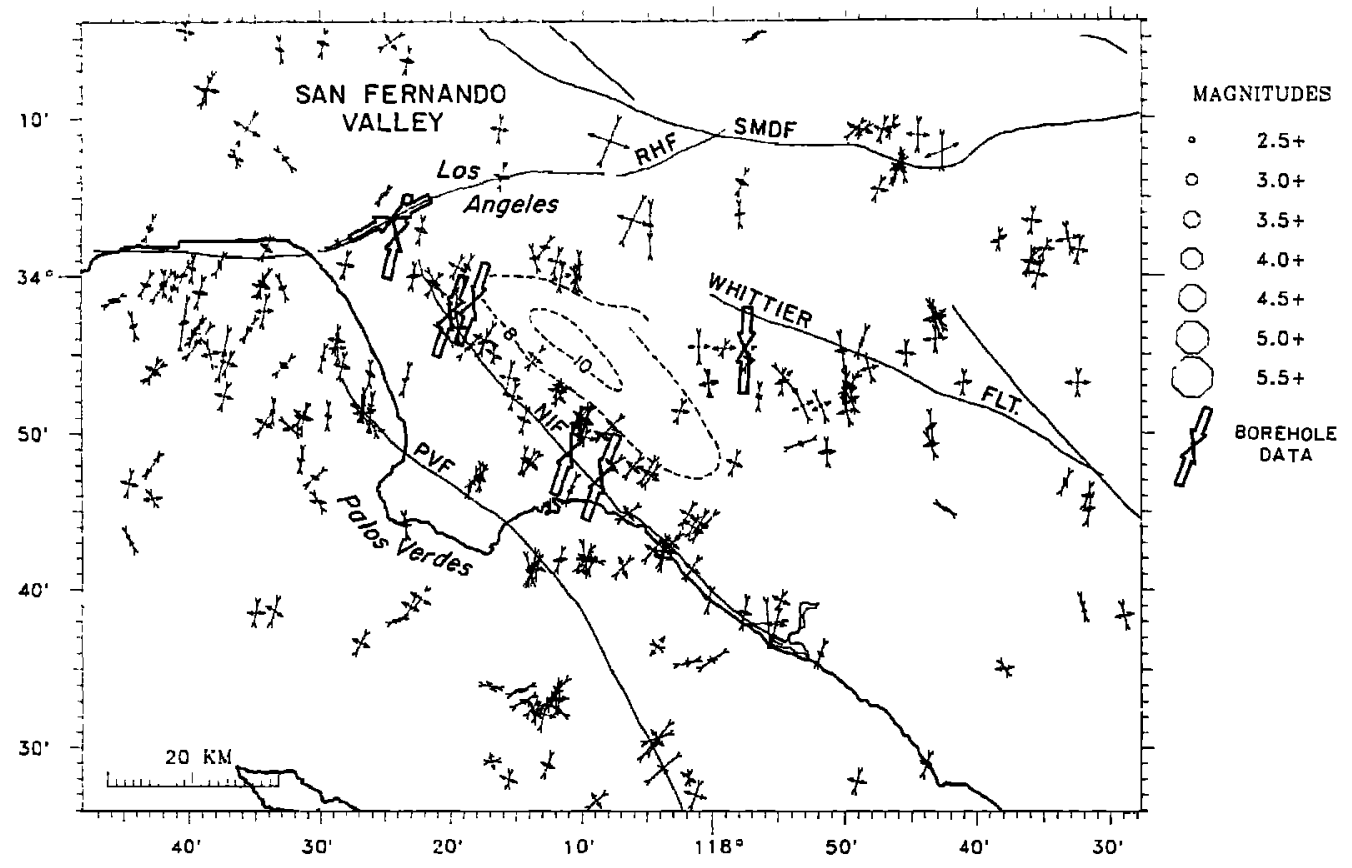

Fig. 15. Map showing $P$ and $T$ axes from the 244 focal mechanisms. Six $P$ axes from borehole breakouts determined by Mount [1989] are shown as open arrows. The $7 \mathrm{~km}$ depth contour of the LA basin is shown for reference.

The $P$ axes show some spatial variations in orientation with mostly northerly trending azimuths along the north and eastern sides of the basin but more northeasterly azimuths in the southwest. A similar variation can be seen in the azimuths of fold axes (Figure 16). The fold axes were grouped into three sets of sliding windows along latitudes $33^{\circ} 42^{\prime}, 33^{\circ} 52^{\prime}$, and $34^{\circ} 02^{\prime}$. These overlapping windows, with a constant radius of $23 \mathrm{~km}$, are spaced 23 and $22 \mathrm{~km}$ apart in longitudinal and latitudinal direction, respectively. Azimuths of fold axes within these windows plotted in rose diagrams show how fold axes in the north and the eastern parts of the basin have azimuths that differ about $30^{\circ}-40^{\circ}$ from azimuths of fold axes in the southwestern part of the basin.

The $P$ and $T$ axes or fold axes alone, however, do not adequately represent the local stress field, because the trend of the $P$ axes can differ from the maximum principal stress by $45^{\circ}$ [McKenzie, 1969]. To determine the average deviatoric stress field in the Los Angeles basin, the data set of focal mechanisms was inverted for the orientations of the principal stresses and their relative magnitude. Furthermore, to determine spatial variations in the deviatoric stress field across the basin the data set was divided up into 15 spatial groups and each group was inverted separately.

\section{The Whole Data Set}

The stereonet plots in Figure 17 show the data and the directions of the maximum principal stresses with $95 \%$ confidence limits obtained from the inversion (see also Table 3). As discussed earlier these stresses were calculated by selecting one of the two nodal planes from each focal mechanism as the actual fault plane. The $95 \%$ confidence limits were calculated assuming that $30 \%$ of the planes were picked incorrectly. The average stress state in the Los
Angeles basin has a maximum horizontal principal stress with an azimuth of $13^{\circ} \pm 3^{\circ}$ and a vertical intermediate stress axis. The value of $\emptyset=0.22$ shows that the relative magnitudes of the three principal stresses are all different, with $S_{2}-S_{3}$ smaller than $S_{1}-S_{3}$ indicating a small but significant thrust faulting component. This stress state reflects the coexistence of strike-slip and thrust faulting regime in the basin. This is also consistent with the prominent late Quaternary strike-slip faults and the strikeslip faulting observed for more than half of the focal mechanisms. This stress state is also consistent with previous results obtained for the Newport-Inglewood fault [Hauksson, 1987], Santa Monica Bay [Hauksson and Saldivar, 1989], and San Fernando aftershocks [Gephart and Forsyth, 1984]. This stress state also fits within the average stress trajectories of central and southem Califormia determined from borehole breakouts by Mount [1989].

\section{Spatial Stress Variations}

To determine the spatial distribution of stress within the Los Angeles basin, the slip vectors from the 244 focal mechanisms were grouped into three sets of sliding windows, like the fold axes, along latitudes $33^{\circ} 42^{\prime}, 33^{\circ} 52^{\prime}$, and $34^{\circ} 02$ '. These overlapping windows, with a constant radius of $23 \mathrm{~km}$, are spaced 23 and $22 \mathrm{~km}$ apart in longitudinal and latitudinal direction, respectively. The overlapping windows provide increased stability of the stress inversion and some smoothing of the stress field. The stereonet projections of the selected planes and slip vectors for each window are shown in Figure 18. Each projection is plotted at the origin of the circle of $23 \mathrm{~km}$ radius that was used to select data for the inversion. The orientations of the maximum principal stress axes and the associated $95 \%$ confidence limits determined assuming $30 \%$ 


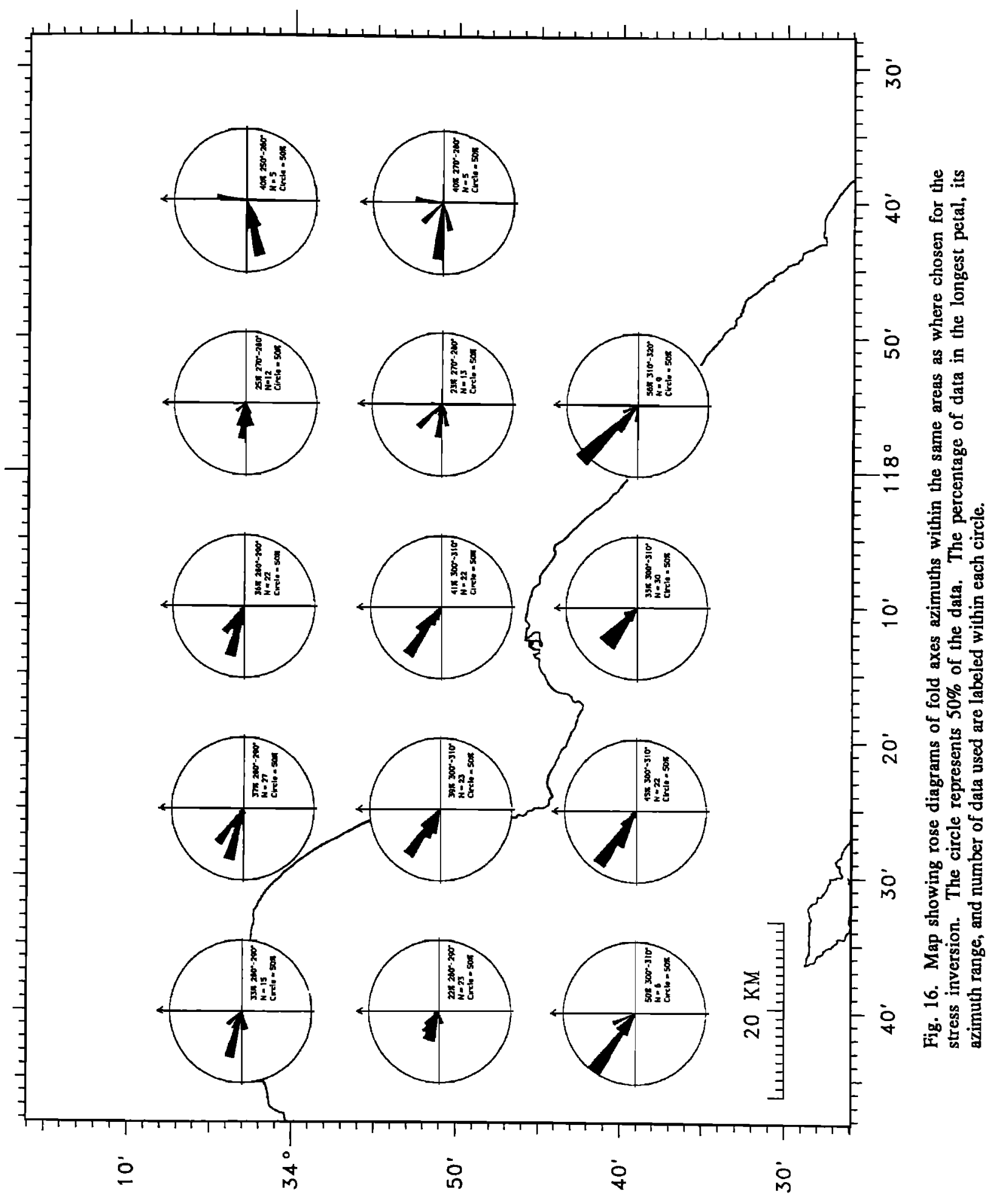



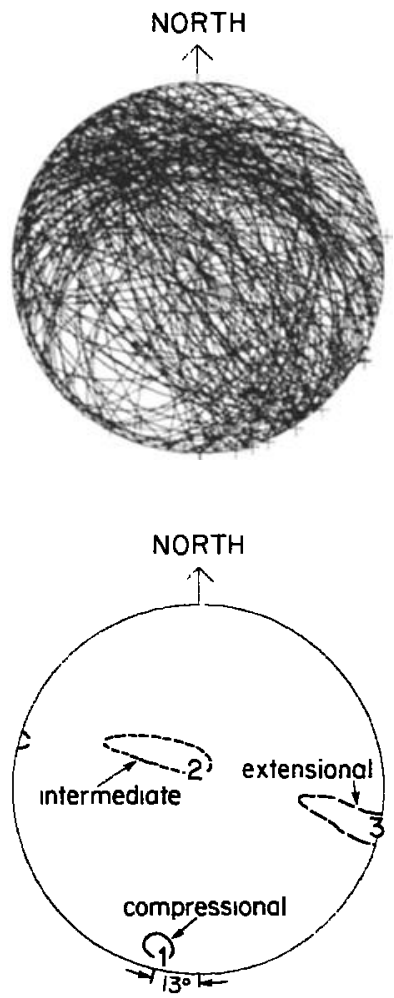

of the planes were picked incorrectly are shown for the 15 data sets in Figure 19 and listed in Table 3. The large misfit angles from the stress inversions for events in the southwestern part of the basin (Table 3 ) are caused mostly by the normal faulting mechanisms. This suggests that the normal faulting mechanisms are caused by localized spatial variations in the stress field and further supports the interpretation that the normal faulting reflects local geometric readjustments.

The state of stress varies significantly across the Los Angeles basin. The most important spatial variation in the state of stress is the variation from $\mathrm{N}^{\circ} \mathrm{W}$ to $\mathrm{N} 31^{\circ} \mathrm{E}$ in the trend of the maximum principal stress. The east side of the basin has a nearly north trending maximum principal stress. From east to west, along the northern edge of the basin the principal stress rotates to the east from $0^{\circ}$ to $13^{\circ}$. This is also consistent with the orientation of the fold axes along the northern flank.

The $32^{\circ}$ variation in azimuth of the maximum principal stress, from a north trending axis to a northeast trending axis, occurs between the northeast basin and the southem segment of the Newport-Inglewood fault and the San Pedro Bay. This deviation is statistically significant because the 95\% confidence limits do not overlap, and it shows that a different stress state exists in the southwest part of the Los Angeles basin. Fold axes in San Pedro Bay have a more northwesterly trend than observed elsewhere in the Los Fig. 17. Data from the 244 focal mechanisms and results from Angeles basin. The agreement between average trends of
the stress inversion. (a) Lower hemisphere projection of all fold axes (shown as petals in Figure 19) and the trend of selected nodal planes (one from each focal mechanisms). the minimum horizontal principal stress suggests that the Location of the slip vector is shown on each nodal plane as a current stress field has existed over the lifetime of the folds plus symbol (with a normal component) or a star (with a thrust or since 2-4 Ma [e.g., Davis et al., 1989].

component). (b) The orientations of the principal stress axes with $95 \%$ confidence areas, determined assuming that $30 \%$ of the planes were picked incorrectly, indicated with solid, heavy dashed or light dashed lines; 1,2 , and 3 are the maximum, intermediate, and minimum principal stress axes.
The second most important spatial variation in the stress field is the variation in $\emptyset$ or the relative magnitude of $S_{2}$ and $S_{3}$, which shows where strike-slip faulting and thrust faulting dominate in the basin (Table 3 and Figure 19). In

TABLE 3. Stress Inversion Results for the Los Angeles Basin

\begin{tabular}{|c|c|c|c|c|c|c|c|c|c|c|c|}
\hline \multirow[t]{2}{*}{$\begin{array}{c}\text { Center } \\
\text { of } \\
\text { Region }\end{array}$} & & \multirow{2}{*}{$\begin{array}{c}\begin{array}{c}\text { Number } \\
\text { of } \\
\text { Planes }\end{array} \\
\#\end{array}$} & \multirow[t]{2}{*}{$\begin{array}{l}0 \\
-\end{array}$} & \multicolumn{2}{|c|}{$\begin{array}{c}\text { Maximum } \\
\text { Principal } \\
\text { Stress } \\
\end{array}$} & \multicolumn{2}{|c|}{$\begin{array}{c}\text { Intermediate } \\
\text { Principal } \\
\text { Stress } \\
\end{array}$} & \multicolumn{2}{|c|}{$\begin{array}{c}\text { Minimum } \\
\text { Principal } \\
\text { Stress } \\
\end{array}$} & \multicolumn{2}{|c|}{$\begin{array}{c}\text { Average } \\
\text { Misfit Angle } \\
\text { B } \\
\end{array}$} \\
\hline & & & & Trend & Plunge & Trend & Plunge & Trend & Plunge & Mean & S. D. \\
\hline All data & & 244 & 0.22 & $-167^{\circ}$ & $8^{\circ}$ & $102^{\circ}$ & $4^{\circ}$ & $-16^{\circ}$ & $80^{\circ}$ & $30^{\circ}$ & $30^{\circ}$ \\
\hline $34^{\circ} 03^{\prime}$ & $\begin{array}{l}117^{\circ} 40^{\prime} \\
117^{\circ} 55^{\prime} \\
118^{\circ} 10^{\prime} \\
118^{\circ} 25^{\prime} \\
118^{\circ} 40^{\prime}\end{array}$ & $\begin{array}{l}26 \\
40 \\
41 \\
53 \\
38\end{array}$ & $\begin{array}{l}0.49 \\
0.37 \\
0.18 \\
0.08 \\
0.24\end{array}$ & $\begin{array}{l}-180^{\circ} \\
-179^{\circ} \\
-167^{\circ} \\
-168^{\circ} \\
-167^{\circ}\end{array}$ & $\begin{array}{l}2^{\circ} \\
6^{\circ} \\
2^{\circ} \\
1^{\circ} \\
1^{\circ}\end{array}$ & $\begin{array}{r}90^{\circ} \\
90^{\circ} \\
-77^{\circ} \\
101^{\circ} \\
-76^{\circ}\end{array}$ & $\begin{array}{r}9^{\circ} \\
9^{\circ} \\
12^{\circ} \\
30^{\circ} \\
44^{\circ}\end{array}$ & $\begin{array}{r}-77^{\circ} \\
-56^{\circ} \\
94^{\circ} \\
-76^{\circ} \\
101^{\circ}\end{array}$ & $\begin{array}{l}80^{\circ} \\
79^{\circ} \\
78^{\circ} \\
59^{\circ} \\
46^{\circ}\end{array}$ & $\begin{array}{l}22^{\circ} \\
25^{\circ} \\
22^{\circ} \\
22^{\circ} \\
21^{\circ}\end{array}$ & $\begin{array}{l}18^{\circ} \\
21^{\circ} \\
19^{\circ} \\
21^{\circ} \\
17^{\circ}\end{array}$ \\
\hline $33^{\circ} 51^{\prime}$ & $\begin{array}{l}117^{\circ} 40^{\prime} \\
117^{\circ} 55^{\prime} \\
118^{\circ} 10^{\prime} \\
118^{\circ} 25^{\prime} \\
118^{\circ} 40^{\prime}\end{array}$ & $\begin{array}{l}32 \\
50 \\
73 \\
65 \\
51\end{array}$ & $\begin{array}{l}0.37 \\
0.34 \\
0.25 \\
0.22 \\
0.17\end{array}$ & $\begin{array}{r}-1^{\circ} \\
9^{\circ} \\
-163^{\circ} \\
-166^{\circ} \\
-170^{\circ}\end{array}$ & $\begin{array}{l}5^{\circ} \\
1^{\circ} \\
9^{\circ} \\
1^{\circ} \\
5^{\circ}\end{array}$ & $\begin{array}{r}90^{\circ} \\
99^{\circ} \\
105^{\circ} \\
-76^{\circ} \\
98^{\circ}\end{array}$ & $\begin{array}{r}19^{\circ} \\
8^{\circ} \\
14^{\circ} \\
2^{\circ} \\
15^{\circ}\end{array}$ & $\begin{array}{r}-106^{\circ} \\
-87^{\circ} \\
17^{\circ} \\
82^{\circ} \\
-63^{\circ}\end{array}$ & $\begin{array}{l}70^{\circ} \\
82^{\circ} \\
80^{\circ} \\
88^{\circ} \\
74^{\circ}\end{array}$ & $\begin{array}{l}22^{\circ} \\
28^{\circ} \\
27^{\circ} \\
24^{\circ} \\
26^{\circ}\end{array}$ & $\begin{array}{l}20^{\circ} \\
29^{\circ} \\
27^{\circ} \\
25^{\circ} \\
24^{\circ}\end{array}$ \\
\hline $33^{\circ} 39^{\circ}$ & $\begin{array}{l}117^{\circ} 40^{\prime} \\
117^{\circ} 55^{\prime} \\
118^{\circ} 10^{\prime} \\
118^{\circ} 25^{\prime} \\
118^{\circ} 40^{\prime}\end{array}$ & $\begin{array}{l}11 \\
34 \\
71 \\
32 \\
10\end{array}$ & $\begin{array}{l}0.34 \\
0.47 \\
0.30 \\
0.18 \\
0.33\end{array}$ & $\begin{array}{l}-179^{\circ} \\
-149^{\circ} \\
-152^{\circ} \\
-159^{\circ} \\
-178^{\circ}\end{array}$ & $\begin{array}{r}6^{\circ} \\
15^{\circ} \\
14^{\circ} \\
17^{\circ} \\
19^{\circ}\end{array}$ & $\begin{array}{r}88^{\circ} \\
120^{\circ} \\
-61^{\circ} \\
107^{\circ} \\
87^{\circ}\end{array}$ & $\begin{array}{r}18^{\circ} \\
3^{\circ} \\
3^{\circ} \\
11^{\circ} \\
11^{\circ}\end{array}$ & $\begin{array}{r}-71^{\circ} \\
20^{\circ} \\
41^{\circ} \\
-15^{\circ} \\
-31^{\circ}\end{array}$ & $\begin{array}{l}71^{\circ} \\
75^{\circ} \\
76^{\circ} \\
70^{\circ} \\
68^{\circ}\end{array}$ & $\begin{array}{l}26^{\circ} \\
42^{\circ} \\
36^{\circ} \\
40^{\circ} \\
30^{\circ}\end{array}$ & $\begin{array}{l}14^{\circ} \\
41^{\circ} \\
32^{\circ} \\
44^{\circ} \\
25^{\circ}\end{array}$ \\
\hline
\end{tabular}




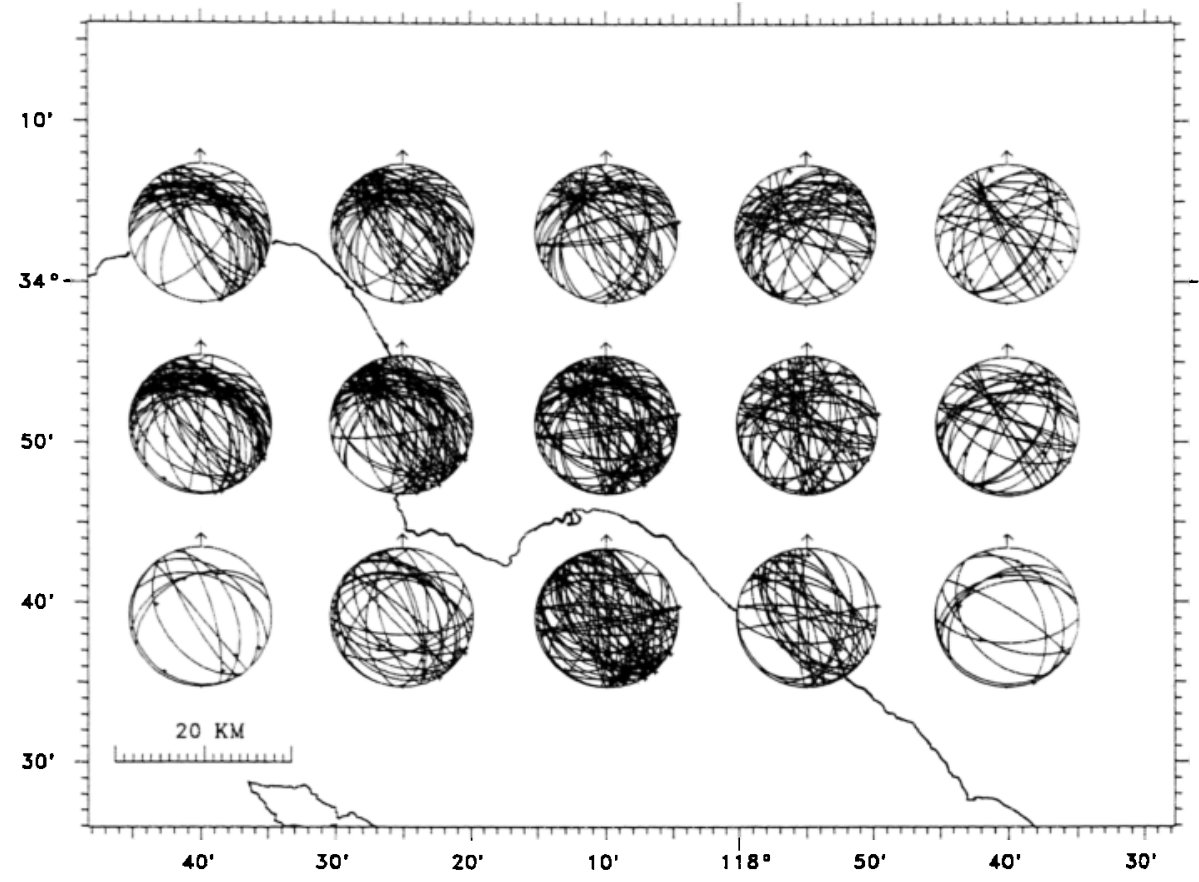

Fig. 18. Map showing the 15 data sets inverted for the stress field. See also caption of Figure 17a.

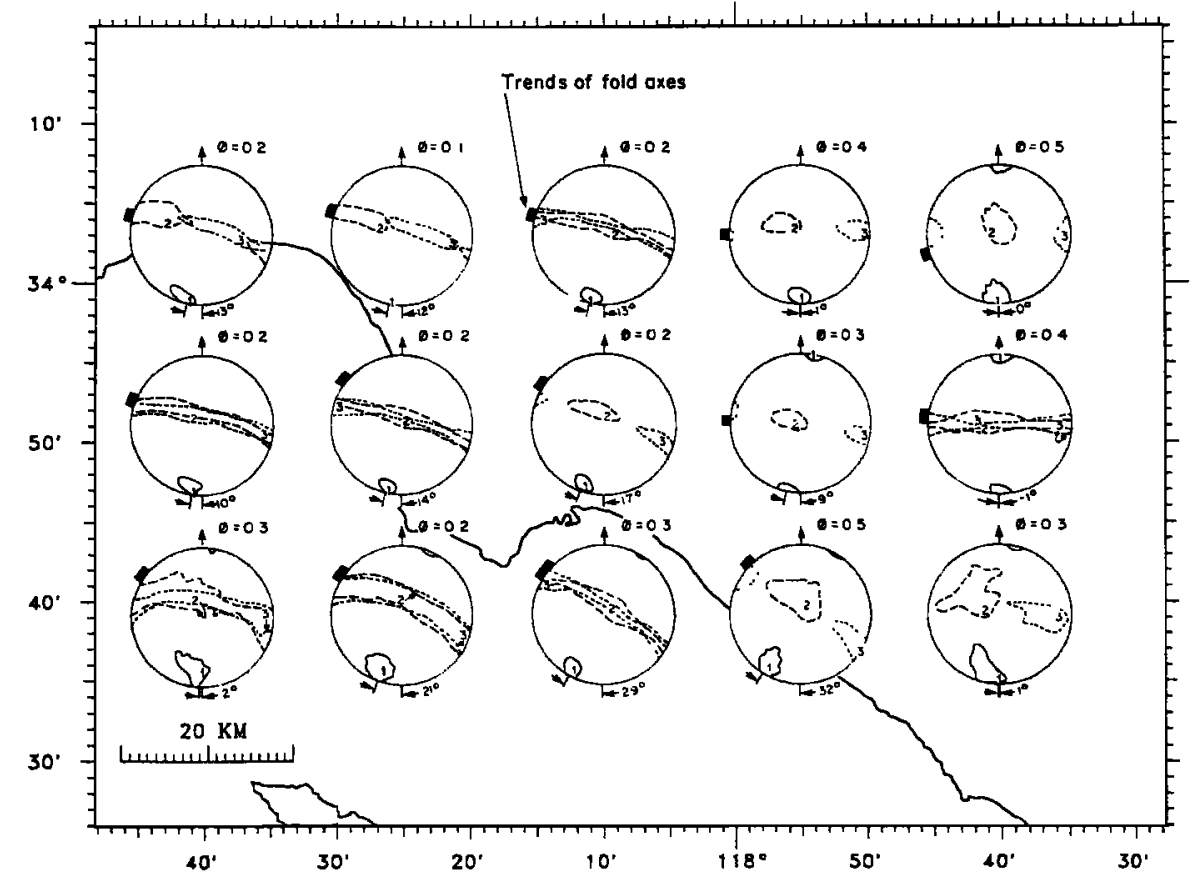

Fig. 19. Map showing the stress field in stereonet projection at the 15 sites. The largest petals from each of the rose diagrams in Figure 16 are also shown as petals on the outside of each stereonet circle. See also caption of Figure $17 b$.

the central part of the basin and along its eastern edge, $0=0.25-0.49$ (i.e., $S_{2}$ is significantly larger than $S_{3}$ ), and the plunge of $S_{2}$ is close to $90^{\circ}$ so that the stress state is equivalent to strike-slip faulting. Along the Elysian Park and the Torrance-Wilmington fold and thrust belt, $\phi=0.08$ -
0.25 (i.e., $S_{2}$ is approximately equal in magnitude to $S_{3}$ ), so the stress state is equivalent to thrust faulting. These spatial variations in the magnitudes and orientations of the principal stresses suggest that the flanks of the basin locally influence the state of stress. 


\section{Discussion}

The Los Angeles basin is not a passive depositional sedimentary basin but a region of complex tectonics [e.g., Yerkes et al., 1965; Davis et al., 1989]. This complexity has made it difficult to interpret the ongoing tectonic deformation and the associated earthquake activity, which, in turn, has resulted in "surprise" earthquakes causing significant property damage [e.g., Hauksson et al., 1988]. This study has contributed toward understanding the complex tectonics through mapping of fold and thrust belts using geologic data and earthquake focal mechanisms and suggesting that the coexistence of strike-slip and thrust faults is the result of decoupling of oblique fault motion.

\section{Fold and Thrust Belts}

The 1987 Whittier Narrows earthquake confirmed that the thrust fault identified by Davis et al. [1989] beneath the Elysian Park anticline is an active fault. Other damaging earthquakes such as the Coalinga and Kettleman Hills earthquakes in central Califormia have also confirmed a causal relationship between folding and faulting [Stein and King, 1984; Eaton, 1990]. In this study, the focal mechanisms of small and moderate-sized earthquakes (2.5 $\leq M \leq 5.9$ ) are used to infer the zones of thrust faulting. If the focal mechanisms show thrust faulting and are located in an area of folding, a belt of thrust faulting and folding is inferred.

Two major fold and thrust belts have been recognized along the flanks of the basin (Figure 11). In the past the folds were ascribed to wrench faulting along the major strike-slip faults [e.g., Wilcox et al., 1973]. The thrust focal mechanisms from this study and the fault-bend and fault-propagation folding models by Suppe [1985] and others indicate that these folds are not associated with strike-slip faults but are caused by movement on blind or concealed thrust faults at depth. This interpretation is also similar to the conclusions of Davis et al. [1989], who used geological data and folding models to interpret the folds in the basin.

Segmentation of fold and thrust belts can limit the size of earthquake rupture and is thus important for evaluating the earthquake potential of these concealed faults. The available data can not resolve whether the fold and thrust belts are underlain by contiguous or segmented faults. Furthermore, the seismicity from 1977 to 1989 is a snap shot in time and may not illuminate all of the relevant concealed fault structures. Several lines of evidence, however, suggest that the thrust belts may be underlain by segmented faults.

The Elysian Park fold and thrust belt is approximately $100 \mathrm{~km}$ long and based on limited evidence can be divided into at least five large segments (Figure 11). The geological and earthquake data alone cannot resolve if each of these large segments are underlain by one continuous thrust fault or many small thrust faults. The segment boundaries are identified from (1) the eastern and western limits of the aftershock zone of the 1987 Whittier Narrows earthquake; (2) the Yorba Linda seismicity trend; and (3)

the intersection with the Newport-Inglewood fault where the depth of the hypocenters appears to be offset with greater focal depths found to the east.
The Torrance-Wilmington fold and thrust belt is approximately $60 \mathrm{~km}$ long and can be divided into three large segments mostly based on the boundaries of oil fields within the belt. Like the Elysian Park thrust zone whether each of the large segments consists of one large or many small thrust faults cannot be resolved. One of the segmentation boundaries is in San Pedro Bay where the Wilmington oil field is offset from the Beta oil field. (Wright [1987] shows the detailed locations of the oil fields.) The other is offshore from Torrance, at the western end of the Torrance oil field. This segmentation boundary is supported by some strike-slip focal mechanisms near the north offshore extension of the Palos Verdes fault. The Torrance-Wilmington and Elysian Park fold and thrust belts appear to merge in Santa Monica Bay and continue to the west beyond Point Dume as one fold and thrust belt [see also, Davis et al., 1989].

Any of these eight large segments could rupture in a moderate-sized $(M>5)$ or large $(M>6.7)$ earthquake. If several segments could rupture simultaneously, a large $(M>6.7)$ or great $(M>7.8)$ earthquake could result. The compressional tectonics of the Los Angeles basin are similar to the tectonics of the southern San Joaquin Valley with concealed thrust faults beneath thick folded sediments. The $1952\left(M_{S}=7.7\right)$ Kem County earthquake ruptured a 75 km-long concealed fault with three distinct segments near the southern end of the San Joaquin Valley [Stein and Thatcher, 1981]. Although the concealed faults in the basin appear to be segmented, a large earthquake that would break multiple fault segments beneath the Los Angeles basin cannot be precluded.

\section{Decoupling of Fault Slip}

Decoupling of fault motion or slip partitioning implies that two sets of faults with distinctly different fault motion coexist and together accommodate oblique fault motion in the same region. Decoupling of fault motion has not been thought important in the past because short faults often exhibit oblique slip and focal mechanisms of small earthquakes showing oblique faulting are also common. However, unlike the small earthquakes in the Los Angeles basin, the moderate and large earthquakes rarely exhibit oblique faulting (Figure $6 b$ ). Recent studies of the San Andreas fault in central California infer that slip is decoupled near the fault such that strike-slip movement takes place on the main trace of the fault and thrust or normal on smaller subsidiary faults [Zoback et al., 1987; Mount and Suppe, 1987; Mount, 1989]. Similarly, along the San Andreas fault in southern California, Jones [1988] found a good correspondence between off-fault earthquakes, late Quaternary tectonic deformation, and state of stress. This suggests that the decoupling of strike-slip from normal and thrust motion in some cases is an important part of faulting.

The Los Angeles basin is a boundary zone between the strike-slip faulting of the Peninsular Ranges to the south and the thrust or reverse faulting of the Transverse Ranges. The merging of these two modes of deformation requires some form of oblique deformation. However, oblique faulting is not observed on mappable surficial faults such as the Whittier and Newport-Inglewood faults where the 
vertical component is less than $10 \%$ of the horizontal component of motion. Rather, the results of this study suggest that for moderate-sized or large earthquakes the fault slip is decoupled into a strike-slip component on steeply dipping northwest to north striking faults and a thrust faulting component on north dipping, east striking faults. Hence these two different types of faulting need to coexist in the basin.

\section{Active Tectonics}

A schematic drawing of the major late Quaternary faults and the fold and thrust belts (Figure 20) summarizes the complex tectonics of the Los Angeles basin. The maximum horizontal principal stresses acting on the basin (determined in this study) and, to the north, on the Mojave segment of the San Andreas fault [Jones, 1988] are also included. The complex tectonics responding to this regional stress field show (1) right-lateral motion on northwest to north-northwest striking faults such as the Whittier and Newport-Inglewood faults; (2) left-lateral motion on east-northeast to northeast striking faults such as the Raymond fault and Yorba Linda trend; (3) thrust motion on west to west-northwest striking gently dipping faults located within the fold and thrust belts; and (4) reverse motion on exposed reverse frontal faults such as the Sierra Madre fault. The strike-slip faults accommodate lateral movements of blocks while the thrust and reverse faults accommodate uplift and crustal shortening.

Weldon and Humphreys [1986] model the deformation of southern California as dominated by lateral motion of crustal blocks to the northwest and west around the "Big
Bend" of the San Andreas fault. Their model explains the difference in convergence across the central Transverse Ranges (about $3 \mathrm{~mm} / \mathrm{yr}$ ) and the western Transverse Ranges (about $20 \mathrm{~mm} / \mathrm{yr}$ ) by left stepping a zone of rightlateral shear separate from the San Andreas across the western Transverse Ranges. The strike-slip movement in the Los Angeles basin is compatible with their model. The Newport-Inglewood and the Whittier faults accommodate northwestward right-lateral motion subparallel to the plate motion of the Pacific plate. The Yorba Linda trend and the Raymond fault accommodate some of the westward left-lateral motion required for the westward motion of these blocks around the "Big Bend".

The Weldon and Humphreys [1986] model includes motions on strike-slip and reverse faults. The results of this study compliment their model by showing how the motion on strike-slip and exposed reverse faults are merged through decoupled fault motion in areas where these faults abut. Weak faults or communication through elastic strain are possible mechanisms for decoupled faulting, which allow the needed oblique motion to be accommodated by the two distinct sets of faults.

\section{Conciusions}

The analysis of 244 single-event focal mechanisms of earthquakes in the Los Angeles basin and available data on folding shows coexistence of major strike-slip and thrust faults. Two fold and thrust belts are identified. The Elysian Park fold and thrust belt extends along the east and north flank of the basin from the north end of the Elsinore

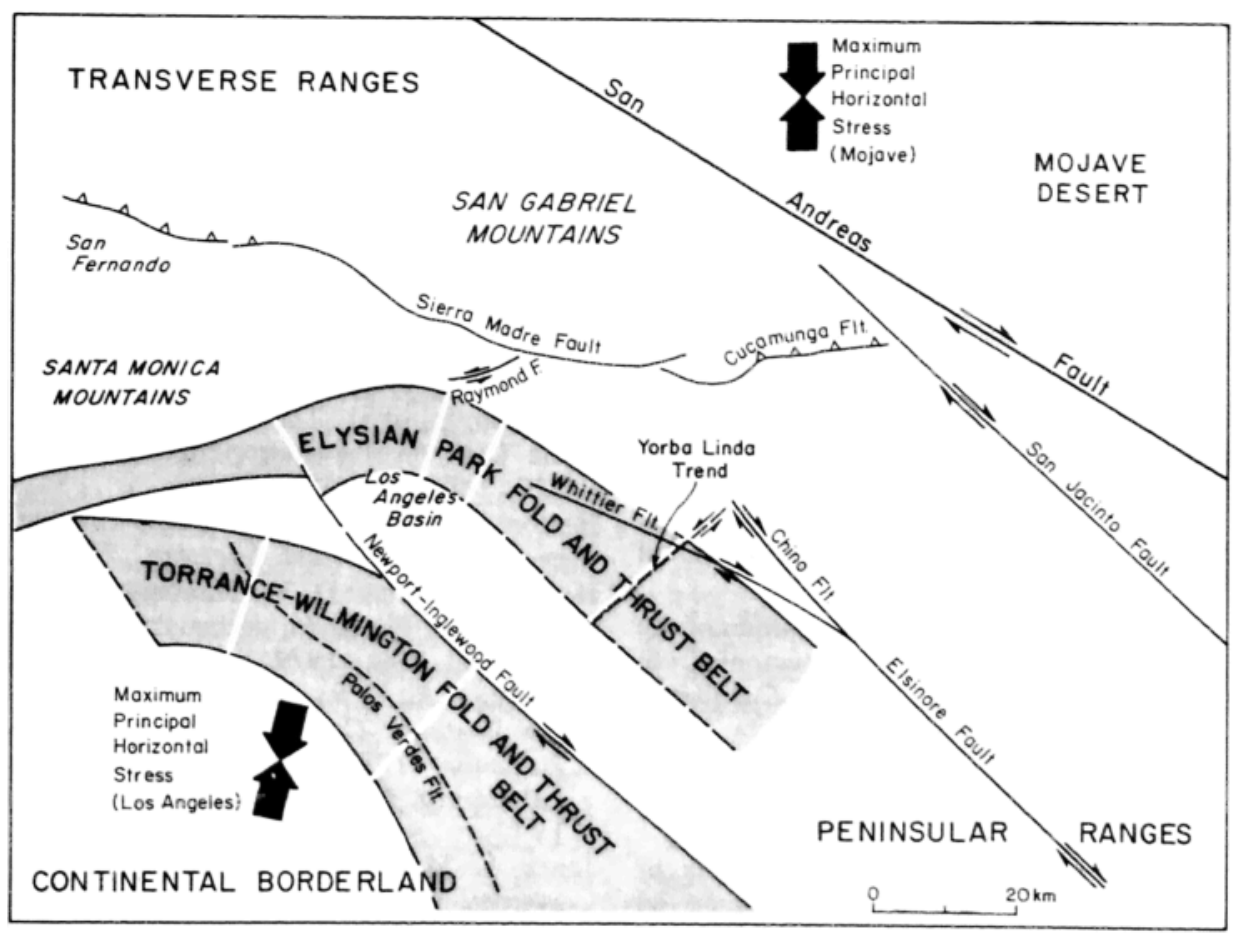

Fig. 20. Schematic tectonic map of the Los Angeles basin showing mappable surficial late Quaternary faults [Ziony and Jones, 1989] and the Yorba Lind seismicity trend. The Elysian Park and Torrance-Wilmington fold and thrust belts are shown as shaded areas. Maximum compressive horizontal principal stresses from this study and Jones [1988] for the Mojave segment of the San Andreas fault are also shown with thick arrows. 
fault, through the Whittier Narrows, beneath downtown Los Angeles, and into Santa Monica Bay. The TorranceWilmington fold and thrust belt extends along the southwest flank of the basin from offshore Newport Beach across the Palos Verdes Peninsula and into Santa Monica Bay. The merging of the strike-slip faulting of the Peninsular Ranges and the thrust faulting of the Transverse Ranges does not occur through oblique faulting but rather through slip partitioning or decoupled strike-slip and thrust faulting. Moderate-sized damaging earthquakes have occurred on both the strike-slip and the thrust faults. The flanks of the basin that surround the less active deep central basin appear to accommodate most of the deformation. The stress field determined from focal mechanisms is consistent with stress directions derived from borehole breakout data. The spatial coincidence of the minimum principal stress directions and the trends of fold axes suggests that the present stress field has existed since $2-4$ Ma. Furthermore, evaluations of the earthquake potential that in the past only took into account the mappable surficial faults also have to take into account the concealed thrust faults.

Acknowledgments. I would like to thank Lucile Jones, Ray Weldon, Thom Davis, Andy Michael and an anonymous reviewer for critical review of the manuscript. I also thank Lily Hsu for help with picking the first motions. I would also like to thank Tom Wright, Lucile Jones, Mason Hill, and John Suppe for interesting discussions. This research was supported by USGS grants 14-08-0001-G1328 and G1761, and USGS cooperative agreement 14-08-0001-A0264.

\section{REFERENCES}

Allen, C. R., P. St. Amand, C. F. Richter, and J. M. Nordquist, Relationship between seismicity and geologic structure in the southem California region, Bull. Seismol. Soc. Am., 55, 753-797, 1965.

Atwater, T., Plate tectonic history of the northeast Pacific ocean and westem north America, in DNAG Vol. $N$, The Northeastern Pacific Ocean and Hawaii, edited by E. L. Winterer, D. M. Hussong, and R. W. Decker, pp. 21-72, Geological Society of America, Boulder, Colo., 1989.

Bryant, M. E., and D. L. Fife, The Peralta Hills fault, a Transverse Ranges structure in the northern Peninsular Ranges, southern California, in Geology and Mineral Wealth of the California Transverse Ranges, edited by D. L. Fife and J. A. Minch, pp. 403-409, South Coastal Geological Society, Santa Ana, Calif., 1982.

Campbell, R. H., and R. F. Yerkes, Cenozoic evolution of the Los Angeles basin area-Relation to plate tectonics, in Aspects of the Geologic History of the California Continental Borderland, Misc. Publ. 24, edited by D. G. Howell, pp. 541-558, Pacific Section, American Association of Petroleum Geologists, Los Angeles, Calif., 1976.

Corbett, E. J., Seismicity and crustal structure studies of southern California: Tectonic implications from improved earthquake locations, Ph.D. thesis, 231 pp., Calif. Inst. of Technol., Pasadena, 1984.

Crook, R., Jr., C. R. Allen, B. Kamb, C. M. Payne, and R. J. Proctor, Quaternary geology and seismic hazard of the Sierra Madre and associated faults, westem San Gabriel Mountains, in Recent Reverse Faulting in the Transverse
Ranges, California, edited by D. M. Morton and R. F. Yerkes, US. Geol. Surv. Prof. Pap., 1339, 27-64, 1987.

Darrow, A. C., and P. J. Fischer, Activity and earthquake potential of the Palos Verdes fault, technical report, $90 \mathrm{pp}$., U.S. Geol. Surv., Menlo Park, Calif., 1983.

Davis, T. L., J. Namson, and R. F. Yerkes, A cross section of the Los Angeles area: Seismically active fold and thrust belt, the 1987 Whittier Narrows earthquake, and earthquake hazard, J. Geophys. Res., 94, 9644-9664, 1989.

DeMets, C., R. G. Gordon, S. Stein, and D. F. Argus, A revised estimate of Pacific-North America motion and implications for western north America plate boundary zone tectonics, Geophys. Res Lett., 14, 911-914, 1987.

Eaton, J. P., Regional seismic background of the May 2, 1983 Coalinga earthquake, in Proceedings of Workshop XXVII, Mechanics of the May 2, 1983 Coalinga Earthquake, edited by M. J. Rymer and W. L. Ellsworth, U.S. Geol. Surv. OpenFile Rep., 85-44, 44-60, 1985.

Eaton, J. P., The May 2, 1983 Coalinga earthquake and its aftershocks from May 2 through Sept. 30, 1983, in The Coalinga, California, Earthquake of May 2, 1983, edited by M. Rymer and W. Ellsworth, U. S. Geol. Surv. Prof. Pap., 1487 , in press, 1990.

Gephart, J. W., and D. W. Forsyth, An improved method for determining the regional stress tensor using earthquake focal mechanism data: Application to the San Fernando earthquake sequence, $J$. Geophys. Res., 89, 9305-9320, 1984.

Gutenberg, B., C. F. Richter, and H. O. Wood, The earthquake in Santa Monica Bay, Califomia, on August 30, 1930, Bull. Seismol. Soc. Am., 22, 138-154, 1932.

Harding, T. P., and A. C. Tuminas, Interpretation of footwall (lowside) fault traps sealed by reverse faults and convergent wrench faults, Am. Assoc. Pet. Geol. Bull., 72, 738-757, 1988.

Hauksson, E., Seismotectonics of the Newport-Inglewood fault zone in the Los Angeles basin, southem Califomia, Bull. Seismol. Soc. Am., 77, 539-561, 1987.

Hauksson, E., and L. M. Jones, The 1987 Whittier Narrows earthquake sequence in Los Angeles, southern California: Seismological and tectonic analysis, J. Geophys. Res., 94, 9569-9590, 1989.

Hauksson, E., and G. V. Saldivar, The 1930 Santa Monica and the 1979 Malibu, California, earthquakes, Bull. Seismol. Soc. $A m ., 76,1542-1559,1986$.

Hauksson, E., and G. V. Saldivar, Seismicity and active compressional tectonics in Santa Monica Bay, southem California, J. Geophys. Res., 94, 9591-9606, 1989.

Hauksson, E., et al., The 1987 Whittier Narrows earthquake in the Los Angeles metropolitan area, Califomia, Science, 239, 1409-1412, 1988.

Heaton, T. H., The 1971 San Fernando earthquake: A double event?, Bull. Seismol. Soc. Am., 72, 2037-2062, 1984.

Heaton, T. H., and D. V. Helmberger, Generalized ray models of the San Fernando earthquake, Bull. Seismol. Soc. Am. 69, 1311-1341, 1979.

Hileman, J. A., C. R. Allen, and J. M. Nordquist, Seismicity of the Southern California Region 1 January 1932 to 31 December 1972, 401 pp., technical report, Seismological Laboratory, Califormia Institute of Technology, Pasadena, 1973.

Jones, L. M., Foreshocks (1966-1980) in the San Andreas system, California, Bull. Seismol. Soc. Am., 74, 13611380, 1984.

Jones, L. M., Focal mechanisms and the state of stress on the San Andreas fault in southem California, J. Geophys. Res., 93, 8869-8891, 1988.

Jones, L. M., K. E. Sieh, E. Hauksson, and L. K. Hutton, The December 3, 1988 Pasadena earthquake: Evidence for strike- 
slip motion on the Raymond fault, Bull Seismol. Soc. Am. 80, 474-482, 1990.

Junger, A., and H. C. Wagner, Geology of the Santa Monica and San Pedro basins, California Continental Borderland, scale 1:250,000, U.S. Geol. Surv. Misc. Field Stud. Map, MF-820, 5 sheets, 1 pamphlet, 1977.

Klein, F. W., User's guide to HYPOINVERSE, a program for VAX and PC350 computers to solve for earthquake locations, US. Geol. Surv. Open-File Rep., 85-515, 24 pp., 1985.

Lsmar, D. L., Structural evolution of the northern margin of the Los Angeles basin, Ph.D. thesis, 142 Pp. Univ. of Calif., Los Angeles, 1961.

Lamar, D. L., Right-slip on Whittier fault system, southern California, in Field Guide of Whittier Fault, edited by E. Gath, in press, Southern California Section, Association of Engineering Geologists, Los Angeles, 1990.

Lin, J., and R. Stein, Coseismic folding, earthquake recurrence, and the 1987 source mechanism at Whittier Narrows, Los Angeles basin, Califomia J. Geophys. Res., 94, 9614-9632, 1989.

McKenzie, D. P., The relation between fault plane solutions for earthquakes and directions of the principal stress, Bull. Seismal. Soc. Am., 59, 591-601, 1969.

Michael, A. J., Determination of stress from slip data: Faults and folds, $J$. Geophys. Res., 89, 11,517-11,526, 1984.

Michael, A. J., Use of focal mechanisms to determine stress: A control study, J. Geophys. Res., 92, 357-368, 1987a.

Michael, A. J., Stress rotation during the Coalinga aftershock sequence, J. Geophys. Res., 92, 7963-7979, $1987 b$.

Morton, D. M., and R. F. Yerkes, Recent reverse faulting in the Transverse Ranges, California, in Recent Reverse Faulting in the Transverse Ranges, California, edited by D. M. Morton and R. F. Yerkes, U.S. Geol. Surv. Prof. Pap., 1339, 1-4, 1987.

Mount, V. S., State of stress in California and a seismic structural analysis of the Perdido fold belt northwest Gulf of Mexico, 220 pp., Ph. D. thesis, Princeton Univ., Princeton, N. J., 1989.

Mount, V. S., and J. Suppe, State of stress near the San Andreas fault: Implications for wrench tectonics, Geology, IS, 1143-1146, 1987.

Nabelek, J., Geometry and mechanism of faulting of the 1980 El Asnam, Algeria, earthquake from inversion of teleseismic body waves and comparison with field observations, $J$. Geophys. Res., 90, 12713-12728, 1985.

Namson, J. S., and T. L. Davis, Seismically active fold and thrust belt in the $S a n$ Joaquin Valley, central Califomia, Geol. Soc. Am. Bull., 100, 257-273, 1988.

Nardin, T. R., and T. L. Henyey, Pliocene-Pleistocene diastrophism of Santa Monica and San Pedro shelves, California continental borderland, Am. Assoc. Pet. Geol. Bull., 62, 247-272, 1978.

Nicholson, C., L. Seeber, P. Williams, and L. R. Sykes, Seismicity and fault kinematics through the eastern Transverse Ranges, California: Block rotation, strike-slip faulting, and low-angle thrusts, J. Geophys. Res., 91, 4891$4908,1986$.

Page, R., Aftershocks and microaftershocks of the great Alaska earthquake of 1964, Bull. Seismol. Soc. Am., 58, 1131-1168, 1968.

Ramsey, J. G., and M. I. Huber, The Techniques of Modern Structural Geology, vol. 2, Folds and Fractures, 700 pp., Academic Press, San Diego, Calif., 1987.

Reasenberg, P., and D. Oppenheimer, FPFIT, FPPLOT and FPPAGE: Fortran computer programs for calculating and displaying earthquake fault-plane solutions, U.S. Geol. Surv. Open-File Rep. 85-739, 46 pp., 1985.
Richter, C. F., Elementary Seismology, 768 pp., W. H. Freeman, New York, 1958.

Roecker, S., and W. L. Ellsworth, VELEST, Fortran Program, U.S. Geol. Surv., Menlo Park, Calif., 1978.

Sanders, C. O., I, Seismotectonics of the San Jacinto fault zone and the Anza Gap, II, Imaging the shallow crust in volcanic areas with earthquake shear waves, $\mathrm{Ph}$. $\mathrm{D}$. thesis, 180 pp., Calif. Inst. of Technol., Pasadena, 1986.

Scholz, C. H., The frequency-magnitude relation of microfracturing in rock and its relation to earthquakes, Bull. Seismol. Soc. Am., 58, 399-415, 1968.

Schol, C. H., The critical slip distance for seismic faulting, Nature, 336, 761-763, 1988.

Stein, R. S., and G. C. P. King, Seismic potential revealed by surface folding: 1983 Coalinga, California, earthquake, Science, 224, 869-872, 1984.

Stein, R. S., and W. Thatcher, Seismic and aseismic deformation associated with the 1952 Kern County, Califomia, earthquake and relationship to the Quatemary history of the White Wolf fault, J. Geophys. Res., 86, 4913-4928, 1981.

Stierman, D., and W. Ellsworth, Aftershocks of the February 21, 1973 Point Mugu, California, earthquake, Bull. Seismol. Soc. Am., 66, 1931-1952, 1976.

Suppe, J., Principles of Structural Geology, 537 pp., PrenticeHall, Englewood Cliffs, N. J., 1985.

Taber, S., The Inglewood earthquake in southem California, June 21, 1920, Bull. Seismol. Soc. Am., 10, 129-145, 1920.

Weldon, R. J., and E. Humphreys, A kinematic model of southern California, Tectonics, 5, 33-48, 1986.

Wetmiller, R. J., R. B. Homer, H. S. Hasegawa, R. G. North, M. Lamontagne, D. H. Weichert, and S. G. Evans, An analysis of the 1985 Nahanni earthquakes, Bull. Seismol. Soc. Am., 78, 590-616, 1988.

Whitcomb, J. H., C. R. Allen, J. D. Garmany, and J. A. Hileman, San Fernando earthquakes series, 1971: Focal mechanisms and tectonics, Rev. Geophys., 11, 693-730, 1973.

Wilcox, R. E., T. P. Harding, and D. R. Seely, Basic wrench tectonics, AAPG Bull., 57, 74-96, 1973.

Wood, H. O., Preliminary report on the Long Beach earthquake, Bull. Seismol. Soc. Am., 23, 43-56, 1933.

Woodward-Clyde Consultants, Report on the evaluation of maximum earthquake and site ground motion parameters associated with the offshore zone of deformation, San Onofre Nuclear Generating Station: report prepared for Southem California Edison, 56 pp., Los Angeles, Calif., 1979.

Wright, T. L., Geologic summary of the Los Angeles basin, in Petroleum Geology of Coastal Southern California, Guidebook, pp. 21-31, Pacific Section, American Association of Petroleum Geologists, Los Angeles, Calif., 1987.

Wright, T. L., Structural geology and tectonic evolution of the Los Angeles basin, in Active Margin Basins, edited by K. T. Biddle, AAPG Memoir, in press, 1990.

Yerkes, R. F., Geologic and seismologic setting, in Evaluating Earthquake Hazards in the Los Angeles Region: An Earth-Science Perspective, edited by J. I. Ziony, U.S. Geol. Surv. Prof. Pap., 1360, 25-41, 1985.

Yerkes, R. F., T. H. McCulloh, J. E. Schoellhamer, and J. G. Vedder, Geology of the Los Angeles basin California-An introduction, U.S. Geol. Surv. Prof Pap., 420-A, A1-A57. 1965.

Ziony, J. I., and L. M. Jones, Map showing late Quaternary faults and 1978-84 seismicity of the Los Angeles region, Califomia, U. S. Geol. Surv. Misc. Field Stud. Map, MF1964, 1989. 
Ziony, J. I., and R. F. Yerkes, Evaluating earthquake and surface-faulting potential, in Evaluating Earthquake Hazards in the Los Angeles Region: An Earth-Science Perspective, edited by J. I. Ziony, U.S. Geol. Surv. Prof. Pap., 1360, 43$91,1985$.

Zoback, M. D., M. L. Zoback, V. S. Mount, J. Suppe, J. P. Eaton, J. H. Healy, D. Oppenheimer, P. Reasenberg, L. M. Jones, C. B. Raleigh, I. G. Wong, O. Scotti, and C. Wentworth, New evidence on the state of stress of the San Andreas fault system, Science, 238, 1105-1111, 1987.
E. Hauksson, Seismological Laboratory, Division of Geological and Planetary Sciences, California Institute of Technology, Pasadena, California, 91125
(Received September 17, 1989; revised January 30, 1990; accepted March 30, 1990.) 